Proceedings of Symposia in Pure Mathematics

Volume 27, 1975

\title{
LINEARIZATION STABILITY OF NONLINEAR PARTIAL DIFFERENTIAL EQUATIONS
}

\author{
ARTHUR E. FISCHER ${ }^{\prime}$ AND JERROLD E. MARSDEN'
}

0. Introduction. In this article we study solutions to systems of nonlinear partial differential equations that arise in riemannian geometry and in general relativity. The systems we shall be considering are the scalar curvature equations $R(g)=\rho$ and the Einstein equations Ric $\left({ }^{(4)} g\right)=0$ for an empty spacetime. Here $g$ is a riemannian metric and $R(g)$ is the scalar curvature of $g, \rho$ is a given function, (4) $g$ is a Lorentz metric on a 4-manifold and Ric $\left({ }^{(4)} g\right)$ denotes the Ricci curvature tensor of (s) $g$.

To study the nature of a solution to a given system of partial differential equations, it is common to linearize the equations about the given solution, solve the linearized equations, and assert that the solution to these linearized equations can be used to approximate solutions to the nonlinear equations in the sense that there exists a curve of solutions to the full equations which is tangent to the linearized solution. This assertion, however, is not always valid. In our study of the above equations we give precise conditions on solutions guaranteeing that such an assertion is valid-at these solutions, the equations are called linearization stable. We also give examples of solutions which are not linearization stable. Although such solutions are exceptional, they still point up the need to exercise caution when such sweeping assumptions are made.

The term "stable" has the general meaning that a stated property is not destroyed when certain perturbations are made, as in structural stability or dynam-

\footnotetext{
AMS (MOS) subject classifications (1970). Primary 53C25, 53C50, 58B20, 83C05; Secondary 35B20, 35J60, 35J70, 58F10, 83Cl0, 83Cl5.

IPartially supported by NSF grants GP-39060 and GP-15735.
}

$Q$ 1975, American Mathematical Society 
ical (Liapunov) stability. For linearization stability the persistent property is "finding solutions in a given direction". If the equations are linearization stable, this property is not destroyed when we pass from the linearized equations to the nonlinear ones by adding on the "higher order terms".

The general set-up is as follows: let $X$ and $Y$ be Banach spaces or Banach manifolds of maps over a compact manifold $M$, and let $\Phi: X \rightarrow Y$ be a given map, e.g., a nonlinear differential operator between $X$ and $Y$; we assume $\Phi$ itself is a differentiable map. Thus for $y_{0} \in Y$

$$
\Phi(x)=y_{0}
$$

is a system of partial differential equations.

Let $T_{x} X$ denote the tangent space to $X$ at $x \in X$ and let

$$
D \Phi(x)=T \Phi(x): T_{x} X \rightarrow T_{\Phi(x)} Y
$$

be the derivative of $\Phi$ at $x$. Thus to each solution $x_{0}$ of (1),

$$
D \Phi\left(x_{0}\right) \cdot h=0, \quad h \in T_{x_{0}} X
$$

is the associated system of linearized equations about $x$, and a solution $h \in T_{x_{0}} X$ to (2) is an infinitesimal deformation (or first order deformation) of the solution $x_{0}$ to (1).

Working in charts, the sum $x_{0}+h$ is then to first order also a solution to (1), since

$$
\begin{aligned}
\Phi\left(x_{0}+h\right) & =\Phi\left(x_{0}\right)+D \Phi\left(x_{0}\right) \cdot h+\text { higher order terms } \\
& =y+\text { higher order terms. }
\end{aligned}
$$

We now ask if there exist a $\delta>0$ and a curve $x(\lambda),|\lambda|<\delta$, of exact solutions of (1), $\Phi(x(\lambda))=y_{0}$ which is tangent to $h$ at $x_{0}$; i.e., such that $x(0)=x_{0}$ and $x^{\prime}(0)=h$. If there exists such a curve for each solution $h$ to (2) we say that the equations (1) are linearization stable at $x_{0}$; the curve $x(\lambda)$ is a finite deformation tangent to $h$.

We apply our general set-up and procedures to study the linearization stability and instability of the scalar curvature equation in riemannian geometry, and the Einstein empty space field equations of general relativity. We also study the possibility of isolated unstable solutions to these equations.

In the course of proving our results, we shall prove that several subsets of the space of riemannian metrics $\mathscr{H}$ and its cotangent bundle $T^{*} \mathscr{M}$ are actually submanifolds; these submanifolds are of interest to geometers and relativitists.

We summarize our main results as follows:

1. Riemannian geometry. Let $M$ be a a compact $C^{\infty}$ manifold, $\operatorname{dim} M \geqq 2$, and $\rho: M \rightarrow R$ a $C^{\infty}$ map.

I.A. LiNEARIZATION STABILITY. The equation $R(g)=\rho$ is linearization stable about any solution $g_{0}$ if either

(a) $\operatorname{dim} M=2$; 
(b) $\operatorname{dim} M \geqq 3$ and $\rho$ is not a constant $\geqq 0$; or

(c) $\operatorname{dim} M \geqq 3, \rho=0$ and $\operatorname{Ric}\left(g_{0}\right) \neq 0$ (here $\operatorname{Ric}\left(g_{0}\right)$ is the Ricci tensor of $\left.g_{0}\right)$. If $\operatorname{dim} M \geqq 3$, and either

(d) $\rho=$ constant $>0$, and $\left(M, g_{0}\right)$ is a standard $n$-sphere in $R^{n+1}$; or

(e) $\rho=0$ and $g_{0}$ is flat,

then $\boldsymbol{R}(\boldsymbol{g})=\rho$ is not linearization stable around $g_{0}$.

These results occur in suitable $H^{s}, W^{s, p}$, or $C^{\infty}$ finction spaces.

1.B. Submanifolds of $\mathscr{M}$. Let $\mathscr{A}_{\rho}=\{g \in \mathscr{M}: R(g)=\rho\}$ denote the set of riemannian metrics with prescribed scalar curvature. Assume that one of the following conditions is satisfied:

(a) $\operatorname{dim} M=2$;

(b) $\operatorname{dim} M=3, \rho$ is not a positive constant;

(c) $\operatorname{dim} M \geqq 4, \rho$ is not a positive constant, and if $\rho=0$, then there exists a flat riemannian metric on $M$.

Then $\mathscr{H}_{\rho}$ is a $C^{\infty}$ closed submanifold of $\mathscr{M}$.

Also, the space of flat riemannian metrics on $M$ is a $C^{\infty}$ closed submanifold of $\mathscr{M}$, and has the structure of a homogeneous fibre bundle.

I.C. Isolation. If $g_{F} \in \mathscr{F}$, there exists a neighborhood $U_{g,} \subset \mathscr{A}$ such that if $g \in U_{g}$ and $R(g) \geqq 0$, then $g$ is also flat. Consequently, $\mathscr{M}_{0}$ is the disjoint union of the closed submanifolds $\mathscr{H}_{0}-\mathscr{F}$ and $\mathscr{F}$, under the hypothesis that $\mathscr{F} \neq$ $\varnothing$ (not needed if $\operatorname{dim} M=2$ or 3 ).

II. General relativity.

II.A. LINEARIZATION STABILITY OF THE EINSTEIN EQUATIONS. Let $V$ be a spacetime, $\operatorname{dim} V=4$, and ${ }^{(4)} g$ a Lorentz metric satisfying Einstein's empty space equations $\operatorname{Ric}\left({ }^{(4)} g\right)=0$. Let $M$ denote a compact spacelike hypersurface with metric $g$ and second fundamentat form $k$. Assume that $(g, k)$ satisfies conditions

$C_{x}:$ If $k=0$, then $g$ is not flat;

$C_{8}$ : If $L_{X} g=0$ and $L_{X} k=0$, then $X=0$ ( $L_{X}$ denotes the Lie derivative by the vector field $X$ );

$C_{\mathrm{t}}: \operatorname{tr} k$ (= the trace of $k$ ) is constant on $M$.

If $M$ is noncompact, one imposes asymptotic conditions, and we assume

$\mathscr{H}: \mathrm{g}$ is complete and asymptotically flat, $k$ is asymptotically zero:

$\bar{C}_{\mathrm{d}}:$ If $L_{X} g=0$ and $L_{X} k=0$, and $X$ is asymptotically zero, then $X=0$.

Then $\mathrm{Ric}{ }^{\left({ }^{(4)} g\right)}$ is linearization stable on the appropriate maximal Cauchy development of $M$.

II.B. SUBMANIFOLDS OF $T^{*} \mathscr{H}$ AND LINEARIZATION STABILTY OF THE CONSTRAINT Equations. Let $\mathscr{C}_{X}=\left\{(g, \pi) \in T^{*} \cdot \mathscr{H}: \mathscr{H}(g, \pi)=0\right\}$ and $\mathscr{B}_{\delta}=\left\{(g, \pi) \in T^{*} \mathscr{H}: \delta_{g} \pi\right.$ $=0\}$ denote the set of solutions to the Hamiltonian and divergence constraint on a compact spacelike hypersurface $M$. Replace $k$ with $\pi$ in conditions $C_{\dot{x}}, C_{\mathrm{j}}$, and $C_{\mathrm{tr}}$.

11.B.1. The set $\mathscr{C}_{x}=\left(\mathscr{E}_{x}-\mathscr{F} \times\{0\}\right) \cup(\mathscr{F} \times\{0\})$ is the disjoint union of submanifolds of $T^{*} \mathscr{M}$, and $\mathscr{F} \times\{0\}$ is closed. $\mathscr{C}_{\times}$itself, however, need not be a submanifold as $\mathscr{C}_{*}-\mathscr{F} \times\{0\}$ is not closed.

The Hamiltonian constraint equation $\mathscr{H}(g, \pi)=0$ is linearization stable about a 
solution $(g, \pi)$ if and only if $(g, \pi)$ satisfies condition $C_{\not} \leftrightarrow(g, \pi) \notin \mathscr{F} \times\{0\}$.

II.B.2 Let $(g, \pi) \in \mathscr{C}_{\mathrm{d}}$ satisfy condition $C_{\delta}$. Then $\mathscr{C}_{\mathrm{d}}$ is a $C^{\infty}$ submanifold of $T^{*} \mathscr{H}$ in a neighborhood of $(g, \pi)$. The divergence constraint $\delta_{g} \pi=0$ is linearization stable about $a(g, \pi) \in \mathscr{C}_{3}$ if and only if condition $C_{3}$ is satisfied.

II.B.3. The constraint set $\mathscr{C}=\mathscr{C}_{\infty} \cap \mathscr{C}_{s}$ is a $C^{\infty}$ submanifold in a neighborhood of those $(g, \pi) \in \mathscr{C}$ that satisfy conditions $C_{x}, C_{\delta}$, and $C_{t r}$.

II.C. IsOLATON. There are no isolated solutions tot he empty space constraint equations of general relativity. However, the solutions $\mathscr{F} \times\{0\} \subset T^{*} \mathscr{M}$ are isolated among those solutions which also satisfy tr $\pi=0$.

As a consequence of I.C, in a neighborhood $U_{g}$, of a flat metric, (i) there are no metrics with $R(g) \geqq 0$ and $R(g) \neq 0$, and (ii) $R(g)=0$ implies $g$ is flat, so the flat solutions of $R(g)=0$ are isolated solutions. This result extends to a full neighborhood of the flat metrics the "second order version" of these results obtained by Kazdan-Warner [21] and Brill [5]. Our isolation result I.C. was inspired by the work of Brill.

In [6, Brill-Deser obtain to second order similar results for the linearization stability and the isolation problem of the constraint equations, and in [10], [11], Choquet-Bruhat and Deser prove that Minkowski space (which satisfies conditions $\mathscr{A}$ and $\bar{C}_{\delta}$ ) is linearization stable.

The following simple but useful test for proving linearization stability will be our basic technique.

THeOREM. Let $X, Y$ be Banach manifolds and $\Phi: X \rightarrow Y$ be $C^{1}$. Let $x_{0} \in X$ and $\Phi\left(x_{0}\right)=y_{0}$. Suppose that $T \Phi\left(x_{0}\right)$ is surjective with splitting kernel. Then the equation $\Phi(x)=y_{0}$ is linearization stable about $x_{0}$.

Proor. From the implicit function theorem, the set $\Phi^{-1}\left(y_{0}\right)$ is a $C^{1}$ submanifold near $x_{0}$ with tangent space the kernel of $T \Phi\left(x_{0}\right)$. Thus $h \in T_{x_{0}} X$ is a first order deformation iff $h \in \operatorname{ker} T \Phi\left(x_{0}\right)$ iff $h \in T_{x_{1}}\left(\Phi^{-1}\left(y_{0}\right)\right)$, and since $\Phi^{-1}\left(y_{0}\right)$ is a submanifold, there exists a curve $x(\lambda) \in \Phi^{-1}\left(y_{0}\right)$ which is actually tangent to $h$.

Remarks. 1. If $T \Phi\left(x_{0}\right)$ is surjective, then $\Phi$ maps a neighborhood of $x_{0}$ onto a neighborhood of $y_{0}$.

2. In this paper we work with Hilbert manifolds so that the splitting condition is automatic. However, the Banach space context is important as the spaces $W^{s, p}$ are often needed; see 1.2.1, Remark 4.

In the cases of immediate concern, the hard part of the linearization stability problem will be to establish surjectivity of the appropriate map under as minimal assumptions as possible. This is done pretty much on an ad hoc basis by using various elliptic operator methods.

If $T \Phi\left(x_{0}\right)$ is not surjective, the equation $\Phi(x)=y_{0}$ may still be linearization stable about $x_{0}$. This actually happens for the equation $R(g)=\rho, \rho=$ constant $\geqq 0$, on a 2-dimensional manifold $M$; see I.2.3, Remark 1 .

On the other hand, even if $\Phi^{-1}\left(y_{0}\right)$ is a submanifold of $X$, the equation $\Phi(x)=y_{0}$ need not be linearization stable around a solution $x_{0}$.

EXAMPLE 1. Let $\Phi: R^{2} \rightarrow R,(x, y) \mapsto x\left(x^{2}+y^{2}\right)$. Then $\Phi^{-1}(0)=\{(0, y): y \in R\}$ 
$=\{y$-axis $\}$ is a submanifold of $R^{2}$, and $d \Phi(0,0)=0$, so that any $h=\left(h_{1}, h_{2}\right)$ is a solution to the linearized equations $d \phi(0,0) \cdot h=0$. However, if $h_{1} \neq 0, h$ cannot be tangent to any curve $(x(\lambda), y(\lambda)) \in \Phi^{-1}(0)$ of exact solutions of $\Phi(x, y)=0$.

ReMarK. The difficulty in this example is that the tangent space $T_{(0.0)}\left(\Phi^{-1}(0)\right)=$ $R$ is smaller than the formal tangent space $\operatorname{ker}(d \Phi(0,0))=R^{2}$; the formal tangent space therefore contains nonintegrable directions. A similiar phenomenon occurs for the equation $R(g)=0(\operatorname{dim} M \geqq 3)$ which is linearization unstable about a flat solution but whose solution set $\mathscr{H}_{0}$ is a $C^{\infty}$ submanifold; see 1.5.2, Remark 4 .

In the applications we shall see that if equation (1) is not linearization stable at a solution $x_{0}$ and if $h$ is a solution to the linearized equations (2) at $x_{0}$, then it is possible to get an extra condition on $h$ in order that it be tangent to a curve of exact solutions of (1). For suppose $x(\lambda)$ is a curve of solutions to $(1)$ with $\left(x(0), x^{\prime}(0)\right)=$ $\left(x_{0}, h\right)$ and $h$ a solution to (2). Differentiating $\Phi(x(\lambda))=y_{0}$ twice and evaluating at $\lambda=0$ gives

$$
\left.\frac{d \Phi}{d \lambda}(x(\lambda))\right|_{\lambda=0}=\left.D \Phi(x(\lambda)) \cdot x^{\prime}(\lambda)\right|_{\lambda=0}=D \Phi\left(x_{0}\right) \cdot h=0
$$

and

$$
\begin{aligned}
\frac{d^{2} \Phi}{d \lambda^{2}}(x(\lambda)) & =\left.D^{2} \Phi(x(\lambda)) \cdot\left(x^{\prime}(\lambda), x^{\prime}(\lambda)\right)\right|_{\lambda=0}+\left.D \Phi(x(\lambda)) \cdot x^{\prime \prime}(\lambda)\right|_{\lambda=0} \\
& =D^{2} \Phi\left(x_{0}\right) \cdot(h, h)+D \Phi\left(x_{0}\right) \cdot x^{\prime \prime}(0)=0,
\end{aligned}
$$

so that (2) and (3) are necessary conditions on the derivatives $x^{\prime}(0)=h$ and $x^{\prime \prime}(0)$ of a curve $x(\lambda) \in \Phi^{-1}\left(y_{0}\right)$. Condition (2), the linearized equations, is a condition on $h$ alone; (3) is then a condition on $x^{\prime \prime}(0)$ in terms of a solution $h$. If, however, the second term involving $x^{\prime \prime}(0)$ could be made to drop out, (3) might provide a "second order" condition on $h$. For example, if $\Phi: X \rightarrow R$ and $x_{0}$ is a critical - point of $\Phi, d \Phi\left(x_{0}\right)=0$, but $d^{2} \Phi\left(x_{0}\right) \neq 0$, then (3) becomes

$$
d^{2} \Phi\left(x_{0}\right) \cdot(h, h)=0 \text {, }
$$

a second order condition on $h$, which may not be implied by the first order equations (2). In that case solutions $h$ to (2) which do not also satisfy (4) cannot be tangent to any curve $x(\lambda) \in \Phi^{-1}\left(y_{0}\right)$. When this occurs, we say that (4) is an "extra (second order) condition" on $h$.

If $d^{2} \Phi\left(x_{0}\right)=0$, then of course (4) does not generate an extra condition on $h$. However, if (1) is unstable, we can get an extra condition by going to higher order deformations. For example, by considering third order derivatives, we have

$$
\begin{aligned}
\left.\frac{d^{3} \Phi(x(\lambda))}{d \lambda^{3}}\right|_{\lambda=0}= & d^{3} \Phi\left(x_{0}\right) \cdot(h, h, h) \\
& +3 d^{2} \Phi\left(x_{0}\right) \cdot\left(h, x^{\prime \prime}(0)\right)+d \Phi\left(x_{0}\right) \cdot x^{\prime \prime \prime}(0)=0 .
\end{aligned}
$$

Thus if $d \Phi\left(x_{0}\right)=0, d^{2} \Phi\left(x_{0}\right)=0$, but $d^{3} \Phi\left(x_{0}\right) \neq 0$, then there is an extra third order condition

$$
d^{3} \Phi\left(x_{0}\right) \cdot(h, h, h)=0
$$


on first order deformations $h$.

EXAMPLe 2. Let $\Phi: R^{2} \rightarrow R,(x, y) \mapsto x^{2}-y^{2}$. Then $\Phi^{-1}(0)=\{(x, y): y= \pm x\}$, $d \Phi(0,0)=0$, and the extra second order condition on $h=\left(h_{1}, h_{2}\right)$ is $d^{2} \Phi(0,0)$. $(h, h)=2\left(h_{1}^{2}-h_{2}^{2}\right)=0 \Leftrightarrow h_{2}= \pm h_{1}$.

EXAMPLe 3. Let $\phi(x, y)=x^{3}+x y^{2}$ as in Example 1. Then $d \Phi(0,0)=0$ and $d^{2} \phi(0,0)=0$. But we now have a third order condition on $h=\left(h_{1}, h_{2}\right)$,

$$
d: D(0,0) \cdot(h, h, h)=6 h_{1}\left(h_{1}^{2}+h_{2}^{2}\right)=0 \Leftrightarrow h=\left(0, h_{2}\right) \text {. }
$$

REMARKs. 1. If in these examples the extra condition on $h$ is satisfied, then $h$ is actually the tangen: to a curve $(x(\lambda), y(\lambda)) \in \Phi^{-1}(0)$. In our later applications, we will not always know if satisfaction of the extra condition will be sufficient to find a curve $x(\lambda) \in \Phi^{-1}\left(y_{0}\right)$ tangent to $h$; see the end of I.4.

2. In Example 2, when $d^{2} \Phi\left(x_{0}\right) \neq 0$, the third order equations $(S)$ do not provide an extra condition on first order deformations.

In the main applications we have in mind, the following example will be more generic:

EXAMPLE 4. Let $X=H^{s}\left(T_{q}^{f}(M)\right)$ and $Y=H^{s-k}\left(T_{q}^{p}(M)\right)$ be the linear spaces of Sobolev sections of tensor bundles over a compact manifold $M$ with volume element $d \mu$ (see I.1), and let

$$
\Phi: H^{s}\left(T_{q}^{p}(M)\right) \rightarrow H^{s-\alpha}\left(T_{q}^{p}(M)\right)
$$

be a nonlinear differential ope-ator of order $k$. If for $y_{0} \in Y, \Phi(x)=y_{0}$ is not linearization stable about a solution $x_{0} \in X$, then $\gamma_{x_{0}}=D \Phi\left(x_{0}\right)$ is not surjective, and this condition can be expressed vin the Fredholm alternative by the fact that

$$
\text { ker } \gamma_{x_{0}}^{*} \neq\{0\},
$$

where $\gamma_{x_{q}}^{*}: H^{s}\left(T_{q}^{p}(M)\right) \rightarrow H^{s-k}\left(T_{q}^{p}(M)\right)$ is the $L_{2}$-adjoint of $\gamma_{x_{1}}$, defined by

$$
\int_{M}\left(\gamma_{x_{0}}^{*} f\right) \cdot h d \mu=\int_{M} f \cdot\left(\gamma_{x_{0}} h\right) d \mu .
$$

Thus if $f \in$ ker $\gamma_{x_{1}}^{*} f \neq 0$, transvecting (3) with $f$ and integrating over $M$ gives the extra condition on $h$,

$$
\int f D^{2} \Phi\left(x_{0}\right) \cdot(h, h) d \mu=0,
$$

since the term involving $x^{\prime \prime}(0)$,

$$
\int f \cdot\left(D \phi\left(x_{0}\right) \cdot x^{\prime \prime}(0)\right) d \mu=\int\left(\gamma_{x_{1}}^{*} f\right) \cdot x^{\prime \prime}(0) d \mu=0,
$$

drops out for $x^{\prime \prime}(0)$ arbritrary.

REMarks. 1. The extra condition (7) is now an integrated condition, corresponding to the fact that $D \Phi\left(x_{0}\right)^{*}$ is an $L_{2}$-adjoint; i.c. we do not get an extra condition on $h$ until we integrate (3) with $f \in \operatorname{ker}\left(D \Phi\left(x_{0}\right)\right)^{*}$ so that the term involving $x^{\prime \prime}(0)$ drops out. Equation (3) itself is just a pointwise condition on the acceleration $\boldsymbol{x}^{\prime \prime}(0)$ in terms of $h$ and does not lead to an extra condition on $h$. 
2. In this example and in our later applications there will be an "independent" extra condition for each dimension in $\operatorname{ker}\left(D \Phi\left(x_{0}\right)\right)^{*}$, as is evident from (7) (providing (7) really is an extra condition; see Remark 3). Note that in Examples 2 and 3, $\operatorname{ker}(d \Phi(0,0))^{*}=R$, and there is one extra condition on $h$ as expected.

3. It may happen that solutions to the linearized equations automatically satisfy (7) for all $f \in \operatorname{ker} \gamma_{x_{0}}^{*}$; e.g., this occurs when $D \Phi\left(x_{0}\right)$ is not surjective but $\Phi(x)=y_{0}$ is linearization stable. This actually happens for the equation $R(g)=\rho=$ constant $\geqq 0$ when $\operatorname{dim} M=2$. Of course, in these cases we can never find an extra condition on first order deformations $h$ by considering higher order variations of $\Phi(x)$ $=y_{0}$.

4. In certain circumstances, the extra integrated condition implied by linearization instability can be converted to a pointwise condition on first order deformations. This situation signals that an even stronger type of instability is occurring; viz., that $x_{0} \in S \subset \Phi^{-1}\left(y_{0}\right)$, and that $S$ is an isolated subset of solutions; i.c., there exist no solutions near $S$ which are not in $S$. This situation actually occurs in a neighborhood of the flat solutions of $R(g)=0$; see 1.5 .

In Part II of this paper we will consider the problem of linearization stability of the Einstein equations, a system of nonlinear evolution equations. The problem of linearization stability for a nonlinear evolution equation is interesting when there are some nonlinear constraints on the initial data of the form $\phi(x)=y_{0}$. Then linearization stability of $\Phi$ implies that the corresponding evolution equation, ' say

$$
\dot{x}=F(x),
$$

is also linearization stable, if it satisfies suitable uniqueness and existence theorems. Indeed, we can argue formally as follows: let $\phi\left(x_{0}\right)=y_{0}$ and let $x(t)$ satisfy $\dot{x}=F(x), \bar{x}(0)=x_{0}$. Let $h(t)$ satisfy the linearized evolution equations

$$
h(t)=D F(x(t)) \cdot h(t)
$$

with initial condition $h(0)$ satisfying the linearized constraint equation

$$
D \Phi\left(x_{0}\right) \cdot h(0)=0 .
$$

Let $x_{0}(\lambda)$ be a curve through $x_{0}$ with $\left.(d / d \lambda) x_{0}(\lambda)\right|_{\lambda=0}=h(0)$. Now solve (8) with initial data $x_{0}(\lambda)$ to get a one-parameter family of solutions $x(t, \lambda)$,

$$
\dot{x}(t, \lambda)=F(x(t, \lambda)),
$$

with $x(t, 0)=x(t)$ and $x(0, \lambda)=x_{0}(\lambda)$. Then $x(t, \lambda)$ is a curve of solutions of $(8)$ which is tangent to the linearized solution $h(t)$; i.e., $\left(x(t, \lambda), d x(t, \lambda) /\left.d \lambda\right|_{\lambda=0}=(x(t)\right.$, $h(t))$. For by differentiating (10),

$$
\begin{aligned}
\left.\frac{d}{d \lambda} \dot{x}(t, \lambda)\right|_{\lambda=0} & =\left.\frac{d}{d t} \cdot \frac{d}{d \lambda} x(t, \lambda)\right|_{\lambda=0} \\
& =\left.D F(x(t, \lambda)) \cdot \frac{d x}{d \lambda}(t, \lambda)\right|_{\lambda=0}=\left.D F(x(t)) \cdot \frac{d x}{d \lambda}(t, \lambda)\right|_{\lambda=0}
\end{aligned}
$$


and

thus

$$
\left.\frac{d x}{d \lambda}(0, \lambda)\right|_{\lambda=0}=\left.\frac{d x_{0}}{d \lambda}(\lambda)\right|_{\lambda=0}=h(0)
$$

$$
\left.\frac{d x}{d \lambda}(t, \lambda)\right|_{\lambda=0}=h(t)
$$

since both sides satisfy (9) with the same initial conditions.

For these reasons, linearization stability of the Einstein equations reduces to the linearization stability of the constraint equations on the initial Cauchy data $(g, \pi)$ (although in the above argument, one needs uniqueness of solutions for the equations (8) and (9); for the Einstein equations one has uniqueness only up to coordinate-transformation so that a further argument is needed; see II.5). Since these constraint equations involve the scalar curvature rather than the Ricci curvature of a riemannian metric $g$, the linearization stability of the Einstein cquations is similar to the scalar curvature equation in the riemannian case. Thus because of the dynamical aspect of Lorentz manifolds (see e.g. [1], [14]), Ricci-fiat Lorentz manifolds are in some ways more manageable than Ricci-flat riemannian manifolds. For example, we have not been able to establish whether $\operatorname{Ric}(g)=0$ is linearization stable around a nonflat Ricci-flat solution; indeed the existence of complete ricmannian nonflat Ricci-flat metrics is unknown.'

During the course of this work, many people were consulted. We would especially like to thank Professors J. P. Bourguignon, D. Ebin, J. Guckenheimer, R. Palais, A. Weinstein and J. A. Wolf. The final form of Theorem I.2.I was obtained jointly with J. P. Bourguignon and I.3.I with A. Weinstein.

For further details of the topics and results presented in this paper, see [16], [18].

\section{Deformations of Riemannian Structures}

I.1. Some preliminaries. Throughout, $M$ will denote a $C^{\infty}$ compact connected oriented $n$-manifold without boundary, $n \geqq 2, T_{q}^{p}(M)$ the bundle of p-contravariant and $q$-covariant tensors on $M$, and $C^{z}\left(T_{q}^{p}(M)\right)$ the $C^{k}$ sections, $0 \leqq k \leqq \infty$. Let $g_{b}$ be a fixed "background" $C^{\infty}$ riemannian metric on $M$ with associated covariant derivative $\nabla: C^{k}\left(T_{q}^{p}(M)\right) \rightarrow C^{k-1}\left(T_{q+1}^{p}(M)\right)$ and volume element $d \mu_{s}$. Then for sections $t_{1}, t_{2} \in C^{\circ}\left(T_{q}^{p}(M)\right)$ and for integer $s \geqq 0$, we let

$$
\left(t_{1}, t_{2}\right)_{H^{*}}=\sum_{0 \leq \leq \leq s} \int_{\Delta A}\left\langle\nabla^{\prime} t_{1}(x), \nabla^{\prime} t_{2}(x)\right\rangle_{x} d_{\mu_{S}}(x),
$$

the $H^{s}$ inner product on sections $C^{\propto}\left(T_{q}^{p}(M)\right)$. In the above $\nabla^{\prime}=\nabla \circ \nabla \circ \ldots \circ \nabla$ is the covariant derivative iterated $l$ times, and $\langle,\rangle_{x}$ is the pointwise inner product

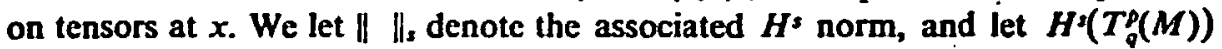
denote the completion of $C^{\wedge x}\left(T_{q}^{p}(M)\right)$ in this norm. Thus $H^{s}\left(T_{q}^{p}(M)\right)$ is the Sobolev space of $H^{s}$ sections. While the norm depends on $g_{b}$, the space and the topology do not.

We will make use of the following two basic Sobolev theorems that are standard in functional analysis (see [27] for more information and references): 
(i) For $s>n / 2+k, H^{\prime}\left(T_{q}^{q}(M)\right) \subset C^{k}\left(T_{q}^{p}(M)\right)$, and $\|t\|_{C} \leqq$ (constant) $\|t\|_{H r}$ for $t \in H^{\prime}\left(T_{q}^{*}(M)\right)$, where

$$
\|t\|_{c}=\sup _{x \in \mathbb{A}}\left\{\|t(x)\|_{x},\|\nabla t(x)\|_{x}, \cdots,\left\|\nabla^{k} t(x)\right\|_{x}\right\} .
$$

(ii) For $s>n / 2, H^{s}$ is a ring under pointwise multiplication; i.e., if $B: T_{q}^{P}(M) \times$ $T_{q}^{p}(M) \rightarrow T_{q^{\prime}}^{p}(M)$ is some pointwise bilinear map, then $B$ induces a continuous bilinear map of the corresponding Sobolev spaces, and

$$
\left\|B\left(t_{1}, t_{2}\right)\right\|_{H S} \leqq \text { const }\left\|t_{1}\right\|_{r r}\left\|t_{2}\right\|_{B P} \text {. }
$$

We shall refer to this property as the Schauder ring property. More generally, if $s>\pi / 2$, multiplication from $H^{*} \times H^{*}$ to $H^{k}$ is continuous, $0 \leqq k \leqq s$.

For $s>n / 2$, we let

$$
\begin{aligned}
& H^{s}=H^{s}(M ; R) \text {, the } H^{s} \text { functions on } M \text {; } \\
& X^{s}=H^{s}\left(T_{0}^{1}(M)\right) \text {, the } H^{s} \text { vector fields; } \\
& S_{2}^{s}=H^{s}\left(T_{2, y m}^{0}(M)\right) \text {, the } H^{s} \text { 2-covariant symmetric tensor fields; } \\
& S_{s}^{2}=H^{s}\left(T_{0,0 \text { m }}^{2}(M)\right) \text {, the } H^{s} \text { 2-contravariant symmetric fields; } \\
& \mathscr{D}^{s+1}=\left\{\text { the group of } H^{s+1} \text { diffeomorphisms on } M\right\} \text {; } \\
& \mathscr{M}^{*}=\mathscr{H}^{0} \cap S_{2} \text {, the space of } H^{s} \text { riemannian metrics on } M \text {, } \\
& \text { where } \mathscr{M}^{0} \text { is the space of continuous riemannian metrics. }
\end{aligned}
$$

$\mathscr{H}^{s} \subset S_{2}^{z}$ is an open convex cone, so that for $g \in \mathscr{M}^{s}, T_{E} \mathscr{M}^{s}=S_{2}^{z}$ and $T . \mathscr{H}^{s}$ $=\mathscr{A}^{3} \times S_{2}$, the tangent bundle of $\mathscr{H}^{s}$.

For $g \in \mathscr{A}^{3}$, we let $d \mu_{k}$ denote the associated volume element.

As in Ebin [13], when the " $s$ " is omitted on any of the above spaces, " $\infty$ " will

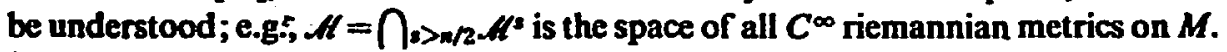

For $g \in \mathscr{H}^{x+1}, X \in \mathscr{X}^{x+1}$, and $h \in S_{2}^{*}$, we let $L_{X} g \in S_{2}^{z}$ be the Lie derivative of $g$ with respect to $X$, and $\delta_{g} h \in X^{3}$ the divergence of $h$. In local coordinates, $L_{X} g$ $=X_{i \mid j}+X_{j i}$ and $\delta_{g} h=-g^{i k} h_{k \mid j}^{\prime}$ (a vertical bar denotes covariant derivative with respect to the metric $g$ ).

Let $\alpha_{g}: \mathscr{X}^{s+1} \rightarrow S_{2}^{s}$ denote the map $X \mapsto L_{x} g$. Using $g$ to define the $L_{2}$ inner products

$$
\begin{aligned}
\left(X_{1}, X_{2}\right) & =\int_{M}\left\langle X_{1}(x), X_{2}(x)\right\rangle_{x} d \mu_{g}(x) \\
\left(h_{1}, h_{2}\right) & =\int_{M}\left\langle h_{1}(x), h_{2}(x)\right\rangle_{x} d \mu_{g}(x)
\end{aligned}
$$

we can see from Stokes' theorem that $\alpha_{g}$ and $2 \delta_{g}$ are adjoints:

$$
\left(\alpha_{g} X, h\right)=2\left(X, \delta_{g} h\right), \quad X \in \mathscr{X}^{s+1}, h \in S_{2}^{s+1} \text {. }
$$

Also $\alpha_{g}$ has injective symbol, so from Berger-Ebin [4] (modified to use an $H^{s+1}$ metric), $S_{2}^{*}$ splits as a $L_{2}$ orthogonal direct sum 


$$
S_{2}^{s}=\grave{S}_{2}^{s} \oplus \alpha_{g}\left(\mathscr{X}^{s+1}\right)
$$

where $\hat{S}_{2}^{s}=$ ker $\delta_{g}=\left\{h \in S_{2}^{s}: \delta_{g} h=0\right\}$, the divergence free symmetric tensors. Thus each $h \in S_{2}^{*}$ can be decomposed as

$$
h=\dot{h}+L_{X g}
$$

where $\delta_{h} \dot{h}=0$ and where the pieces $h$ and $L_{x} g$ are unique and orthogonal in the $L_{2}$ metric. We will refer to this decomposition as the canonical decomposition of $h \in S_{2}^{-}$

Note that the splitting in (1) is not valid if $g$ is only of class $H^{s}$, since then $\alpha_{g}(X)$ $=L_{x g}$ need only be of class $H^{x-1}$. If however $g$ is a flat riemannian $H^{2}$ metric, then the splitting (1) is still valid (see I.3).

In the decomposition (2), the vector field $X$ satisfies

$$
\delta h=\delta L_{X g}=\Delta X+(d \delta X)^{s}-2 \operatorname{Ric}(g) \cdot X
$$

where $(d \delta X)^{*}$ denotes the vector corresponding to the 1 -form $d \delta X$; in general we will let $t^{s}$ denote the totally contravariant form of any tensor $t$, and $t^{b}$ denote the totally covariant form; e.g., if $t$ is of type $\left(\begin{array}{l}1 \\ 2\end{array}\right),\left(t^{5}\right)^{i j k}=g^{i b} g^{k x} t_{j k}^{i k}$ and $\left(t^{b}\right)_{i j k}=g_{i a} t_{j k s}$ $\Delta X=\left((d \delta+\delta d) X^{b}\right)^{*}$ is the Laplace-de Rham operator acting on vector fields, $\delta X=-\operatorname{div} X=-X^{i_{i i}}$ is the divergence of $X, \operatorname{Ric}(g)=R_{i j}$ is the Ricci tensor of $g$ and $\operatorname{Ric}(g) \cdot X=R_{i}^{i} X^{j}$. The above computation uses the contracted Ricci commutation formula $X^{j}{ }_{i \mid j}-X^{j}{ }_{|j| i}=R_{i j} X^{j}$, and the Weitzenböck formula for the Laplace-de Rham operator (sce e.g. Nelson [29]), $\Delta X=\bar{\Delta} X+\operatorname{Ric}(g) \cdot X$ where $\bar{\Delta} X=-g^{j k} X^{i}{ }_{1 / k}$ is the "rough Laplacian" on vector fields. Throughout we use the conventions of Nelson [29] and I ichnerowicz [25].

If $X=\operatorname{grad} \phi$ is a gradient vector field, then $L_{x g}=2$ Hess $\phi=2 \nabla \nabla \phi=2 \phi_{10 j ;}$, the Hessian or double covariant derivative of $\xi$, and $(\Delta X)^{\xi}+d \delta X=\Delta d \psi+d \Delta \psi$ $=2 d \Delta \phi$. Thus we have the identity

$$
\delta \operatorname{Hess} \psi=(d \Delta \phi)^{2}-\operatorname{Ric}(g) \cdot d \psi
$$

which we shall need in the proof of 1.2.1. By contracting the Bianchi identities one also has

$$
\delta \operatorname{Ric}(g)=-\frac{1}{2}\left(d(R(g))^{2}\right.
$$

where $R(g)=R_{i} i_{i}$ is the scalar curvature of $g$.

Finally, as in [13], we let $A: \mathscr{D}^{s+1} \times \mathscr{H}^{s} \rightarrow \mathscr{H}^{3},(\eta, g) \mapsto \eta^{*} g$ denote the right group action of $\mathscr{D}^{s+1}$ on $\mathscr{M}^{s}$ by pullback of metrics (locally this is just coodinate transformation of $g$ by $\eta$ ). Fixing $g \in \mathscr{M}^{s}$, let $\mathcal{O}_{g}=\left\{\eta^{*} g: \eta \in \mathscr{D}^{s+1}\right\}$ be the orbit of $g$. For $g \in \mathscr{H}^{s+1}, \mathscr{C}_{\mathrm{g}}$ is a $C^{1}$ submanifold and $T_{g} O_{g}=\alpha_{g}\left(\mathscr{X}^{s+1}\right)$. Thus in the decomposition of $h \in T_{g} \mathscr{M l}^{3}, h=h+L_{x} g$, the $L_{X g}$ piece is tangent to the orbit which is the direction of isometric changes of $g$, while the orthogonally directed $h$ piece is in the direction of "true" geometric deformationis.

For $g \in \mathscr{H}^{s}$, the orbit map $\psi_{g}: \mathscr{D}^{s+1} \rightarrow \mathscr{H}^{s}, \varphi \mapsto \varphi^{*} g$ is only continuous and need 
not be $C^{1}$. Thus $\mathcal{O}_{g}$ need only be a $C^{0}$ submanifold. This is another reason why the splitting ( $(1)$ is not valid for $g \in \mathscr{M}^{s}$ (unless $g$ is a flat $H^{s}$ metric); however for $g \in$ $\mathscr{M}^{s+1}$, the orbit map $\psi_{g}$ is $C^{\prime}$.

1.2. Deformations of the scalar curvature equation. Let $s>n / 2+1, g \in \mathscr{H}^{s}$ and let $R(g)$ denote the scalar curvature of $g$. Consider $R(\cdot)$ as a map

$$
R(\cdot): \mathscr{M}^{s} \rightarrow H^{s-2}, \quad g \mapsto R(g) .
$$

Since $R(g)$ is a rational combination of $g$ and its first two derivatives, we see from the Schauder ring property that if $s>n / 2+2, R$ is a $C^{\infty}$ map. This is also true for $s>n / 2+l$ if we use the fact that the second derivatives of $g$ occur linearly.

Thus we consider $R(\cdot)$ to be a nonlinear second order differential operator.

For $g \in \mathscr{M}^{\prime}, s>n / 2+1$, we let

$$
r_{g}=D R(g): T_{g} \mathscr{H}^{s} \approx S_{2}^{s} \rightarrow T_{R(g)} H^{s-2} \approx H^{s-2}
$$

denote the derivative of $R(\cdot)$. A classical computation, given for example in [25], shows that for $h \in S_{2}^{z}$

$$
r_{\delta}(h)=\Delta \mathrm{tr} h+\delta \delta h-h \cdot \operatorname{Ric}(g) .
$$

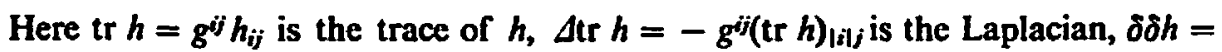
$h^{i j} l_{l, j}$ is the double covariant divergence, $\operatorname{Ric}(g) \in S_{2}^{s-2}$ is the Ricci tensor of $g$, and $h \cdot \operatorname{Ric}(g)=\langle h, \operatorname{Ric}(g)\rangle=g^{j i} g^{a b} h_{i a} R_{j b}$ is the pointwise contraction.

We let $\gamma_{g}^{*}: H^{s} \rightarrow S_{2}^{-2}$ be the $L_{z^{2}}$-adjoint of $\gamma_{g}$, defined by

$$
\int_{M}\left\langle\gamma_{\varepsilon}^{*} f, h\right\rangle d \mu_{g}=\int_{M}\left\langle f, T T_{\varepsilon} h\right\rangle d \mu_{g} .
$$

Using Stokes' theorem, $\gamma_{8}^{*} f$ is easily computed to be

$$
r_{g}^{*} f=g \Delta f+\text { Hess } f-f \operatorname{Ric}(g) .
$$

A somewhat remarkable property of the scalar curvature map is that locally it is almost always a surjection.

1.2.1. THEOREM. Let $g \in \mathscr{M}^{s}, s>n / 2+1$, and suppose that

(1) $R(g)$ is not a positive constant, and .

(2) if $R(g)=0$, then $\operatorname{Ric}(g) \neq 0$.

Then $R(\cdot): \mathscr{H}^{s} \rightarrow H^{s-2}$ maps any neighborhood of $g$ oñto a neighborhood of $R(g)$.

Proor. First assume $s<\infty$. It then suffices by the standard implicit function theorem to show that $\tau_{g}: S_{2}^{s} \rightarrow H^{s-2}$ is surjective. From elliptic theory (see e.g. [4]), this follows if $\tau_{g}^{*}: H^{s} \rightarrow S_{2}^{s-2}$ is injective and has injective symbol. For $\xi_{x} \in$ $T_{x}^{*} M$ (the cotangent space at $x$ ), the symbol $\sigma_{\xi_{0}}\left(\gamma_{g}^{*}\right): R \rightarrow T_{x}^{*} M \otimes_{\text {sym }} T_{x}^{*} M$ $\left(\otimes_{s y m}\right.$ means the symmetric tensor product) is given by $s \rightarrow\left(-g\left\|\xi_{x}\right\|^{2}+\xi_{x} \otimes \xi_{x}\right) s$, $s \in R$, which is clearly injective for $\xi_{x} \neq 0$ and $n \geqq 2$.

To show $r_{8}^{*}$ injective, assume $f \in \operatorname{ker} \gamma_{\delta}^{*}$, so that 
(a)

$$
\tau_{g}^{*} f=g \Delta f+\text { Hess } f-f \operatorname{Ric}(g)=0 .
$$

Taking the trace yields

$$
(n-1) \Delta f=R(g) f \text {. }
$$

First we consider case (2). Thus if $R(g)=0, \Delta f=0$, so $f$ is a constant. Thus from (a), $f \operatorname{Ric}(g)=0$, and since $\operatorname{Ric}(g) \not \equiv 0, f=0$. Thus in case (2), $r_{s}^{*}$ is injective.

Now consider case (1). We shall show that if $f \neq 0, R(g)=$ constant $>0$. Taking the divergence of (a) yields

$$
-d \Delta f+\delta \operatorname{Hess} f+d f \cdot \operatorname{Ric}(g)-f \delta(\operatorname{Ric}(g))=0
$$

(where $d f \cdot \operatorname{Ric}(g)=f_{i j} R_{i}^{\prime}$ ) and using the identities $\delta$ Hess $f-d \Delta f+d f \cdot \operatorname{Ric}(g)=0$, and $\delta \operatorname{Ric}(g)=-(1 / 2) d(R(g))$, (c) reduces to $-(1 / 2) f d(R(g))=0$. If $f$ is never zero, then $d(R(g))=0$ so $R(g)=$ constant, and in fact from (b) an eigenvalue of the Laplacian, and thus $\geqq 0$. If $R(g)=0$, then $f=$ constant $\neq 0 \Rightarrow \operatorname{Ric}(g)$ $=0$, contradicting (2), and $R(g)=$ constant $>0$ contradicts (1). Thus find $x_{0}$ $\in M$ such that $f\left(x_{0}\right)=0$. We must have $d f\left(x_{0}\right) \neq 0$; indeed, if $d f\left(x_{0}\right)=0$, let $\gamma(t)$ be a geodesic starting at $x$, and let $h(t)=f(r(t))$. Combining (a) and (b) we have

$$
\text { Hess } f=(\operatorname{Ric}(g)-(1 /(n-1)) g R(g)) f \text {. }
$$

Hence $h(t)$ satisfies the linear second order differential equation

$$
\begin{aligned}
h^{\prime \prime}(t) & =\left(\operatorname{Hess} \int_{T^{\prime}(t)} \cdot\left(r^{\prime}(t), r^{\prime}(t)\right)\right. \\
& =\left\{\left(\operatorname{Ric}(g)-(n-1)^{-1} g R(g)\right)_{\tau(t)} \cdot\left(r^{\prime}(t), r^{\prime}(t)\right)\right\} h(t)
\end{aligned}
$$

with $h(0)=0$ and $h^{\prime}(0)=d f(r(0)) \cdot \gamma^{\prime}(0)=0$. Thus $f$ is zero along $\gamma(t)$ and so by the Hopf-Rinow theorem on all of $M$. Thus $d f$ cannot vanish on $f^{-1}(0)$ so 0 is a regular value of $f$ and so $f^{-1}(0)$ is an $n-1$ dimensional submanifold of $M$. Hence $d(R(g))=0$ on an open dense set and hence everywhere. Thus as before $R(g)=$ constant $\geqq 0$, contradicting (1) or (2). Thus if (1) and (2) hold, $f \equiv 0$, $\gamma_{B}^{*}$ is injective, $\gamma_{g}$ is surjective, and $R(\cdot)$ is locally surjective.

The case $s=\infty$ requires some additional arguments. One needs to show that the image neighborhood of $R(\mathrm{~g})$ can be chosen independent of $s$. This is possible because one can construct local right inverses for $R$ by maps independent of $s$; they depend only on the right inverse for the derivative and the geometry of the space. The idea is similar to one-occurring in Ebin [13] and works quite generally when we have $L_{2}$ orthogonal splittings for elliptic operators.

Note. We thank J. P. Bourguignon for pointing out the substantial improvement that $\tau_{s}^{*} f=0, f \neq 0$ implies $R(g)=$ constant. Previously we had condition (1) replaced with the condition $R(g) \leqq 0$. Bourguignon's argument appears in [37].

Remarks. (1) If $\operatorname{Ric}(g)=0$, $\operatorname{ker} \gamma_{s}^{*}=\{$ constant functions on $M\}$, so that $\gamma_{g}^{*}$ is not surjective. It is not known if there exist any nonflat Ricci-flat complete riemannian metrics. If there are none, then condition (2) can be replaced by (2)': If $R(g)$ $=0$, then $g$ is not flat. 
If $\operatorname{dim} M=3$, then $\operatorname{Ric}(g)=0$ implies that $g$ is flat so that (2) can be replaced by (2)'.

(2) If $M=S^{n}$ has the metric $g_{0}$ of a standard sphere of radius $r_{0}$ in $R^{n+1}$, then

$$
\operatorname{Ric}\left(g_{0}\right)=\left(\frac{n-1}{r_{0}^{2}}\right) g_{0} \text { and } R\left(g_{0}\right)=\frac{n(n-1)}{r_{0}^{2}}
$$

so that $f \in$ ker $T_{R_{0}}^{*}$ if

$$
\text { Hess } f=\left(\operatorname{Ric}\left(g_{0}\right)-\frac{1}{n-1} g_{0} R\left(g_{0}\right)\right) f=-\frac{f}{r_{0}^{2}} g_{0} \text {. }
$$

But the eigenfunctions of the Laplacian with first nonzero cigenvalue $n / r_{0}^{2}$ also satisfy Hess $f=-\left(f / r_{0}^{2}\right) g_{0}$. Hence again $\gamma_{B_{0}}$ is not surjective, and ker $\gamma_{B_{0}}^{*}=\left\{f \in H^{s}\right.$ : $\left.\Delta f=\left(n / r_{0}^{2}\right) f\right\}$.

Conversely, a theorem of Obata [31], [32] states that if a riemannian manifold admits a solution $f \neq 0$ of Hess $f=-c^{2} f g$, then the manifold is isometric to a standard sphere in $R^{n+1}$ of radius $1 / c$.

If in Theorem I.2.1., $R(g)=$ constant $>0$ and $\gamma_{g}$ is not surjective, then there exists a solution $f \neq 0$ of

$$
\text { Hess } f=\left(\operatorname{Ric}(g)-(n-1)^{-1} g R(g)\right) f .
$$

This equation is similiar to Obata's equation, and it is reasonable to conjecture that a solution $f \neq 0$ of (e) implies that the space is a sphere. For example if $g$ is an Einstein space, $\operatorname{Ric}(g)=\lambda g$ with $\lambda>0$, then the space is a standard sphere. In fact, if $\operatorname{Ric}(g)$ is parallel (e.g., $g$ is a product of Einstein spaces) then Obata's proof goes through and proves that (e) has solutions only on the standard sphere.

Thus it is reasonable to conjecture that $\gamma_{g}$ is surjective unless $(M, g)$ is flat, or unless $(M, g)$ is a standard sphere. This would be quite a nice result.

(3) Also note that among the spaces with positive constant scalar curvature, if $R(g) /(n-1)$ is not an eigenvalue of the Laplacian, $\Delta f=(R(g) /(n-1)) f$, then $r_{g}$ is surjective.

(4) If $R(g)$ is not a constant $\geqq 0$, then from surjectivity of $D R(g)$, it follows that $R(\cdot)$ is locally surjective in an $H^{s}$ neighborhood of $g$. Thus if $\rho$ is sufficiently near $R(g)$ in the $\mathrm{H}^{s}$ topology, $\rho$ is the scalar curvature of some metric. An analogous theorem holds using $W^{\text {s,p }}$ spaces. In fact, Kazdan and Warner [22] have pointed out that local surjectivity of $R(\cdot)$, together with an approximation lemma, can be used to prove their results concerning what functions can be realized as scalar curvatures for $\operatorname{dim} M \geqq 2$. A variant of this technique yields, for example: $I f n \geqq$ 3 and there is $a g \in \mathscr{H}^{s}$ with $R(g)=0$, Ric $(g) \neq 0$, then $R(\cdot): \mathscr{H}^{s} \rightarrow H^{s-2}$ is surjective; i.e., every function can be realized as a scalar curvature of some metric. This follows from local surjectivity of $R(\cdot)$, together with [22, Theorem C].

(5) Using some recent sharp elliptic estimates of Nirenberg and Walker [30] one can extend much of the above work to noncompact manifolds satisfying suitable asymptotic conditions. We discuss this aspect in [18]. 
For $\rho: M \rightarrow R$ a $C^{\infty}$ map, $s>n / 2+1$, we set

$$
\mathscr{M}_{\rho}^{s}=\left\{g \in \mathscr{M}^{s}: R(g)=\rho\right\} \text { and } \mathscr{M}_{\rho}=\{g \in \mathscr{M}: R(g)=\rho\},
$$

sets of metrics with prescribed scalar curvature $\rho$. Using 1.2.1, we can now prove that under certain mild conditions on $\rho, \mathscr{M}_{\rho}^{s}$ is a $C^{\infty}$ submanifold of $\mathscr{H}^{s}$.

1.2.2. THEOREM. Let $\rho: M \rightarrow R$ be a $C^{\infty}$ map, and $s>n / 2+1$. If either

(a) $\operatorname{dim} M=2$, or

(b) $\operatorname{dim} M \geqq 3$ and $\rho$ is not a constant $\geqq 0$,

then $\mathscr{H}_{\rho}^{s}\left(\right.$ respectively, $\left.\mathscr{H}_{\rho}\right)$ is a $C^{\infty}$ closed submanifold of $\mathscr{A}^{s}$ (respectively, $\left.\mathscr{A}\right)$.

If $\operatorname{dim} M \geqq 3, \rho=0, g \in \mathscr{H}_{0}^{3}$ (respectively, $g \in \mathscr{H}^{0}$ ) and $\operatorname{Ric}(g) \neq 0$ (or if $\operatorname{dim} M=3, g$ is not flat), then $\mathscr{H}_{0}^{s}$ (respectively, $\left.\mathscr{A t}_{0}\right)$ is a $C^{\infty}$ closed submanifold in a neighborhood of $\mathrm{g}$.

Proof. The cases (b) and $\rho=0$ are a direct consequence of the surjectivity of $D R(g)$ and the inverse function theorem.

If $\operatorname{dim} M=2$, we need only consider the case $\rho=$ constant $\geqq 0$ since otherwise $D R(g)$ is surjective. If $\rho=0$, then $\mathscr{A}_{0}^{s}=\mathscr{F}^{s}=$ the set of $H^{s}$ flat riemannian metrics on $M$ \}, which from 1.3 .3 is a $C^{\infty}$ closed submanifold of $\mathscr{M}^{\text {; }}$; similiarly for $\mathscr{H}_{0}=F$.

If $g \in M^{3}$ and $R(g)=\rho=$ constant $>0$, then $(M, g)$ is $H^{3}$ isometric to $\left(S^{2}, g_{0}\right)$, a standard 2-sphere in $R^{3}$ with radius $r_{0}=(2 / \rho)^{1 / 2}$. Thus $\mathscr{M}_{p}^{s}=\mathscr{D}^{s+1}\left(g_{0}\right)=\{g \in$ $\mathscr{M}^{s}: \varphi^{*} g=g_{0}$ for some $\left.\varphi \in \mathscr{D}^{s+1}\right\}=\mathcal{O}_{B \circ}$, the orbit through $g_{0}$, which by [13] is a $C^{\infty}$ closed submanifold of $A^{\prime \prime}$ since $g_{0}$ is $C^{\infty}$; similiarly $\mathscr{H}_{\rho}=\mathscr{D}\left(g_{0}\right)$ is a $C^{\infty}$ closed submanifold of $\mathscr{A}$.

Remarrs. 1. If $R(g)=\rho=$ constant $\geqq 0, \operatorname{dim} M=2, D R(g)$ is not surjective but $\mathscr{M}_{p}$ is still a submanifold, as remarked in the introduction; see also I.2.3, Remark 1 .

2. We are not making any statements about whether $\mathscr{H}_{\rho}^{s}$ is empty or not. This is another question. For example, Lichnerowicz [26] has shown that for spin manifolds with $\vec{A}$ genus not zero, $\mathscr{M}_{\rho}=\varnothing$ if $\rho \geqq 0, \rho \neq 0$. However Kazdan and Warner [21], [22] have shown that if $n \geqq 3$ and $\rho$ is negative somewhere; or if $n=2$ and $\rho$ satisfies a sign condition consistent with the Gauss-Bonnet formula, then $\mathscr{H}^{s} \neq \varnothing$.

3. If $\operatorname{dim} M=1$, then $M=S^{1}$ and $R(g)=0$ for all metrics in $\mathscr{H}^{s}$. Thus, $\mathscr{H}_{\rho}^{s}=\varnothing$ if $\rho \neq 0, \mathscr{H}_{0}^{z}=\mathscr{H}^{\prime z}$, and $R(\cdot)$ is not locally surjective around any $g$.

As another application of 1.2 .1 , we have the following result concerning the linearization stability of the equation $R(g)=\rho$.

I.2.3. TheOREM. Let $g_{0} \in \mathscr{H}^{s}$ (respectively, $g_{0} \in \mathscr{M}$ ), and let $\rho=R\left(g_{0}\right)$. Assume that one of the following conditions is satisfied:

(a) $\operatorname{dim} M=2$;

(b) $\operatorname{dim} M \geqq 3, \rho$ is not a constant $\geqq 0$;

(c) $\operatorname{dim} M \geqq 3, \rho=0$, and $\operatorname{Ric}\left(g_{0}\right) \neq 0$ (or if $\operatorname{dim} M=3, g_{0}$ is not flat).

Then $R(g)=\rho$ is linearization stable about $g_{0}$; i.e. for any $h \in S_{2}^{3}$ (respectively, $h \in S_{2}$ ) satisfying the linearized equations 


$$
D R\left(g_{0}\right) \cdot h=\Delta \operatorname{tr} h+\delta \delta h-h \cdot \operatorname{Ric}\left(g_{0}\right)=0
$$

there exists a $C^{\infty}$ curve $g(\lambda) \in \mathscr{M}_{\rho}^{s}$ of exact solutions of $R(g)=\rho$ such that $(g(0)$, $\left.g^{\prime}(0)\right)=\left(g_{0}, h\right)$.

Proof. Cases (b) and (c) are a consequence of $\mathbf{I . 2 . 1}$ and Theorem 1 in \$0, the case $s=\infty$, as before, requiring a special regularity argument.

Suppose $\operatorname{dim} M=2$; we will show directly that $R(g)=\rho$ is linearization stable about a solution $g_{0} \in \mathscr{A}^{s}$ by showing that we can integrate any first order deformation $h$ to a curve of exact solutions. We need only consider the case $\rho=$ constant $\geqq 0$; otherwise stability follows as in case (b). Thus $\left(M, g_{0}\right)$ is $H^{\text {s }}$ isometric to either a flat torus (if $\rho=0$ ) or a standard 2-sphere $S^{2}$ with radius $r_{0}=(2 / \rho)^{1 / 2}$ (if $\rho>0$ ). If $h \in S_{2}^{s}$, let $h=h+L_{\times 8} g_{0}$ be the canonical decomposition of $h$. Such a decomposition always exists even if $g_{0}$ is only of class $H^{3}$; one decomposes the push-forward of $h$ on the torus or sphere using their $C^{\infty}$ riemannian structures and then pulls back this decomposition; see I.3.

Now suppose $h$ is a solution to the linearized equations,

$$
D R\left(g_{0}\right) \cdot h=D R\left(g_{0}\right) \cdot h=\Delta \operatorname{tr} h-h \cdot \operatorname{Ric}\left(g_{0}\right)=0,
$$

where we have used $D R\left(g_{0}\right) \cdot L_{X g_{0}}=L_{X}\left(R\left(g_{0}\right)\right)=X \cdot d\left(R\left(g_{0}\right)\right)=0$ since $R\left(g_{0}\right)=\rho$ $=$ constant $\geqq 0$ (see also the proof of I.3.4). If $\rho=0, g_{0}$ is fiat; thus $\Delta$ tr $h=0 \Rightarrow$ ir $h=$ constant $\Rightarrow \nabla h=0$, as can be seen by writing out $\delta h=0$ and $d \operatorname{tr} h=0$. But if $h$ is parallel, we can explicitly find a curve $g(\lambda)$ such that $g^{\prime}(0)=h+L_{x} g_{0}$ by the method at the end of I.4.

If $R\left(g_{0}\right)=\rho=$ constant $>0$, then $\operatorname{Ric}\left(g_{0}\right)=\frac{1}{2} \rho g_{0}$, and $D R\left(g_{0}\right) \cdot h=\Delta \operatorname{tr} h-$ $\frac{1}{2} \rho$ tr $h=0 \Rightarrow \Delta$ tr $h=\frac{1}{2} \rho \operatorname{tr} h$. But the first nonzero eigenvalue on the standard sphere is $2 / r_{0}^{2}=\rho$. Thus tr $h=0$.

From the uniformization theorem, any two riemannian metrics on the manifold $S^{2}$ are conformally equivalent; i.e. if $\bar{g}, g \in \mathscr{M}$, then $\bar{g}=\varphi^{\prime \prime}(p g)$ for some $\varphi \in \mathscr{D}$ and $p \in C^{\infty}(M ; R), p>0$. Thus if $h \in T_{g} \mathscr{U}$, and $g(\lambda)$ is a curve tangent to $h, g(\lambda)$ $=\varphi_{\lambda}^{*}(p(\lambda) g), \varphi_{0}=\mathrm{id}_{M,} p(0)=1$. Thus any $h \in T_{g} \not H$ is of the form

$$
h=g^{\prime}(0)=\left.\varphi_{\lambda}^{*}\left(p^{\prime}(\lambda) g+L_{Y}(\rho(\lambda) g)\right)\right|_{\lambda=0}=f g+L_{Y} g, \quad f \in C^{\infty}(M ; R),
$$

which we write as $h=L_{Y g}+(\delta Y) g+\frac{1}{2}(\operatorname{tr} h) g$. Note that $f g$ is not divergence free so that $h=f g+L_{Y g}$ is not the canonical decomposition.

Thus if $\operatorname{tr} h=0$ and $\delta h=0, \delta h=\delta\left(L_{Y g}+(\delta Y) g\right)=0$. But since tr $h=0$,

$$
\begin{aligned}
0 & =\int Y \cdot \delta h d \mu \frac{1}{2} \int L_{Y g} \cdot h d \mu=\frac{1}{2} \int\left(L_{Y g}+(\delta Y) g\right) \cdot h d \mu \\
& =\frac{1}{2} \int h \cdot h d \mu \Rightarrow h=0 .
\end{aligned}
$$

Thus if $\mathrm{tr} h=0$, then the above argument implies that $h=0$. Thus a first order deformation of $R(g)=$ constant $>0$ must be of the form $h=L_{X g_{0}}$ (using the canonical decomposition). Thus if $\varphi_{\lambda}$ is the flow of $X, \varphi_{0}=$ id ${ }_{M}$, the curve $g(\lambda)$ $=\varphi_{\lambda g_{0}} \in \mathscr{H}_{\rho}$ since $R\left(\varphi_{\lambda}^{*} g_{0}\right)=\varphi_{\lambda}^{*}\left(R\left(g_{0}\right)\right)=\left(R\left(g_{0}\right)\right) \circ \varphi_{\lambda}=\rho \circ \varphi_{\lambda}=\rho$, and $\left(g(0), g^{\prime}(0)\right)$ 
$=\left(g_{0}, L_{X g_{0}}\right)$. A similiar argument can be adapted for the $H^{s}$ case.

REMARKS. 1. If $\operatorname{dim} M=2, \rho=$ constant $\geqq 0, R(g)=\rho$ is linearization stable about $g_{0}$ even though $D R(g)$ is not surjective. Note, however, that this is not implied by the submanifold structure of $\mathscr{H}_{p}$; cf. Example 1 of $\$ 0$.

2. If $\operatorname{dim} M \geqq 3$, and $g_{0}$ is flat, then $R(g)=0$ is not linearization stable about $g_{0}$; similarly if $\left(M, g_{0}\right)$ is a standard $n$-sphere $S^{n}, R(g)=\rho=$ constant $>0$ is not linearization stable about $g_{0}$; see I.4.2 and I.4.2, Remark 5. Thus the only case that remains open is whether or not $R(g)=\rho=$ constant $>0$ is linearization stable about a solution $g_{0}$, where $\left(M, g_{0}\right)$ is not a standard $n$-sphere, $n \geqq 3$ or where $g_{0}$ is Ricci-flat but not flat.

3. If $\operatorname{dim} M \geqq 3, R\left(g_{0}\right)=\rho=$ constant $>0$, but $\rho \neq(n-1) \lambda$, where $\lambda$ is a nonzero eigenvalue of $\Delta$, then $R(g)=\rho$ is linearization stable about $g_{0}$; this follows from the proof of 1.2.1, since if $f \in \operatorname{ker} D R\left(g_{0}\right)^{*}, \Delta f=(\rho /(n-1)) f$, so if $\rho /(n-1)$ $\neq \lambda, f=0$ and $D R\left(g_{0}\right)$ is surjective.

1.3. The space of flat Riemannian metrics. In the next section we shall study the set of metrics with zero scalar curvature, $\mathscr{M}_{0}^{s}=\left\{g \in \mathscr{H}^{s}: R(g)=0\right\}$. This case is singular in the sense that the scalar curvature map $R(\cdot)$ fails to be a submersion at those $g$ with $\operatorname{Ric}(g)=0$. Thus $\mathscr{H}_{0}^{s}$ may not be a manifold at these points. This difficulty is investigated in 1.5 and [18].

For $\lambda \in R, s>n / 2+1$, let $\mathscr{E}_{2}^{s}=\left\{g \in \mathscr{A}^{3}: \operatorname{Ric}(g)=\lambda g\right\}$ denote the set of Ein stein metrics with "Einstein constant" $\lambda$. Thus $\mathscr{f}_{0}^{s}=\left\{g \in \mathscr{M}^{3}: \operatorname{Ric}(g)=0\right\}$ is the set of Ricci-flat metrics and is part of the singular set for $R(\cdot)$. From Theorem 1.2.2, $\mathscr{A}_{0}^{z} \mid \mathscr{E}_{0}^{s}$ is a smooth submanifold of $\mathscr{M}^{\prime}$.

We let $F^{3}$ denote the set of $H^{s}$ flat riemannian metrics on $M$, and $\mathscr{P}^{s-1}$ the set of flat riemannian connections. For $\Gamma \in \mathscr{H}^{s-1}$ and $\varphi \in \mathscr{D}^{s+1}\left(=\right.$ the group of $H^{s+1}$ diffeomorphisms of $M$ ), we let $\varphi^{\star} \Gamma \in \mathscr{H}^{s-1}$ denote the pullback of the connection $\Gamma$ by $\varphi$.

Let $\nabla$ be the covariant derivative associated with $\Gamma$ and in local coordinates $x$ on $M$, define the Christoffel symbols $\Gamma_{j k}^{i}$ as usual by

$$
\nabla_{\partial / \partial \times} \frac{\partial}{\partial x_{j}}=\Gamma_{i j}^{k} \frac{\partial}{\partial x^{k}}
$$

Let $\Gamma_{j k}^{i}$ be the Christoffel symbols of the pulled back connection $\Gamma=\varphi^{*} \Gamma$ in the local coordinates $\bar{x}^{j}$ and let $\varphi$ be locally represented by $\bar{x}^{i}\left(x^{j}\right)$. Then pullback of the connection just corresponds to the transformation rules of the Christofrel symbols,

$$
\Gamma_{j k}^{i}=\frac{\partial \bar{x}^{i}}{\partial x^{c}} \frac{\partial x^{a}}{\partial \bar{x}^{j}} \frac{\partial x^{b}}{\partial \bar{x}^{b}} \Gamma_{a s}^{c}-\frac{\partial x^{a}}{\partial \bar{x}^{j}} \frac{\partial x^{b}}{\partial \bar{x}^{b}} \frac{\partial^{2} \bar{x}^{i}}{\partial x^{a} \partial \bar{x}^{b}} \text {. }
$$

The following is a sort of regularity theorem for flat metrics and connections:

I.3.1. ThEOREM. Let $\Gamma \in \mathscr{H}^{s-1}, s>n / 2+1$. Then there exists $a \varphi \in \mathscr{D}^{s+1}$ such that $\varphi^{*} \Gamma \in \mathscr{H}$; i.e., $\varphi^{*} \Gamma$ is a $C^{\infty}$ flat (riemannian) connection. Similarly, if.gF $\mathscr{H}^{*}$, then there exists $a \varphi \in \mathscr{D}^{s+1}$ such that $\varphi^{*} g_{F} \in \mathscr{F}$, the space of $C^{\infty}$ flat riemannian metrics. 
We thank Alan Weinstein for pointing out the following proof. We first prove a local version:

Lemma. Let $\Gamma \in \mathscr{H}^{\mathrm{s}-1}$. Then the coordinate change to normal coordinates is of class $H^{s+1}$. (One could use $C^{k}$ spaces here as well.)

Proof. Let $\Gamma_{j k}^{i}$ be the Christoffel symbols of $\Gamma$ in a coordinate system $x^{j}$, and let $\bar{x}^{i}\left(x^{j}\right)$ be the coordinate change to normal coordinates so that $\Gamma_{j k}^{i}=0$. Thus from the transformation rules for the Christoffel symbols,

$$
\frac{\partial^{2}}{\partial x^{j}} \frac{\bar{x}^{i}}{\partial x^{k}}=\frac{\partial \bar{x}^{i}}{\partial x^{j}} \Gamma_{j k}^{a}
$$

and the Christoffel symbols $\Gamma_{j k}^{a}$ are of class $H^{s-1}$. Now we know by construction of normal coordinates from the exponential map that $\bar{x}^{i}\left(x^{j}\right)$ has the same differentiability as the Christoffel symbols (see e.g. Lang [23, p. 96]); thus $x^{-i}\left(x^{\prime}\right)$ is of class $H^{s-1}$ and $\partial \bar{x}^{i} / \partial x^{a}$ is of class $H^{s-2}$. Thus from multiplication properties of Sobolev spaces (I.1), the right-hand side of (1) is also of class $H^{s-2}$. Thus $\dot{x}^{i}\left(x^{b}\right)$ is actually of class $H^{s}$, and from (1) again is actually of class $H^{s+1}$.

Now we prove the first part of 1.3.1. Using the exponential map we get a new differentiable structure on $M$ in which the connection $r$ is smooth. Call this manifold $M_{1}$. The identity is a map of class $H^{s+1}$ (by the lemma) so can be $H^{s+1}$ approximated by a $C^{\infty}$ diffeomorphism $f: M \rightarrow M_{1}$. Pulling back $\Gamma$ on $M_{1}$ by $f$ gives a $C^{\infty}$ flat connection on $M$ which is $H^{s}$ close and $H^{s+1}$ diffeomorphic to the original connection.

The case of a flat metric can be proved the same way or can be deduced from the result for connections.

This argument shows that if $g$ is of class $H^{3}$ and $\eta^{*} g$ is of class $H^{s}$ and $\eta$ is class $H^{s-1}$ (or just $C^{1}$ ) then $\eta$ is automatically $H^{s+1}$. This result can be used to advantage in the uniqueness problem for the Einstein equations; see Fischer and Marsden [15].

Although it is not known if there exist any complete nonflat Ricci-flat ricmannian manifolds, it is known (Fischer and Wolf [19], [20] ) that a compact manifold cannot admit both flat and nonflat Ricci-flat riemannian metrics. This is cstablished

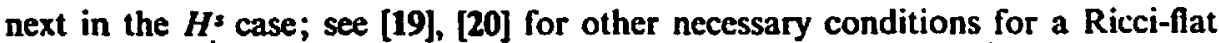
metric to be flat.

1.3.2. TheOREM. If $M$ admits an $H^{*}$ flat riemannian metric $g_{F} \in F^{s}, s>n / 2+1$, then every $g \in \mathscr{M}^{3}$ such that $\operatorname{Ric}(g)=0$ is flat.

ProOr. Let $\varphi \in \mathscr{P}^{s+1}$ be such that $\varphi^{*} g_{F} \in \mathscr{F}$. Then by one of the Bieberbach theorems [35, Theorem 3.3.1], there is a normal riemannian covering $\pi: T^{n} \rightarrow M$ of $M$ by a flat $n$-dimensional torus $T^{m}$, and $\pi$ is a $C^{\infty}$ map.

Now suppose $g \in \mathscr{M}^{s}, \operatorname{Ric}(g)=0$. Then the pulled back metric $\pi^{*} g$ on $T^{n}$ is of class $H^{s}$ and $\operatorname{Ric}\left(\pi^{*} g\right)=\pi^{*}(\operatorname{Ric}(g))=0$. But a Ricci-flat metric on $T^{n}$ is flat [4]; indeed from Hodge's Theorem (using $H^{s}$ metrics), there are $n$ linearly independent 
harmonic vector fields and these are parallel since $\operatorname{Ric}\left(\pi^{*} g\right)=0(\overline{X X}=\Delta X=0 \Rightarrow$ $\nabla X=0)$. Hence $\pi^{*} g$ is flat. Since $\pi$ is a local isometry, $g$ is flat.

Thus if $\mathscr{F}^{s} \neq \varnothing$, then $\mathscr{E}_{0}^{s}=\mathscr{F}^{s}$, so that $\mathscr{M}_{0}^{s}-\mathscr{E}_{0}^{s}=\mathscr{M}_{0}^{s}-\mathscr{F} s$ is a smooth submanifold of $\mathscr{A}^{3}$.

We now show that $\mathscr{F}^{s}$ also is a smooth submanifold of $\mathscr{M}^{s}$; thus if $\mathscr{F}^{\prime} \neq$ $\varnothing, \mathscr{M}_{0}^{s}=\left(\mathscr{M}_{0}^{s}-\mathscr{F}^{s}\right) \cup \mathscr{F}$ is the disjoint union of submanifolds. In I.S, we shall in fact show that $\mathscr{M}_{0}^{s}-\mathscr{F}^{3}$ is closed.

The space $\mathscr{F}^{3}$ (if not empty) has an interesting structure itself.

For $\Gamma \in \mathscr{H}^{s-1}$, let $I_{\Gamma}^{s+1}=\left\{\varphi \in \mathscr{D}^{s+1}: \varphi^{*} \Gamma=\Gamma\right\}$ denote the Lie group of affine transformations of $\Gamma$, and let $\mathscr{F}_{r}^{s}=\{g \in \mathscr{F s}: \Gamma(g)=\Gamma\}$, the set of flat riemannian metrics whose Levi-Civita connection is $\Gamma$. Here we are letting $\Gamma(g)$ designate the Levi-Civita connection of the metric $g$. Thus if $g \in \mathscr{M}^{s}, \Gamma(g)$ is an $H^{s-1}$ connection, and if $\varphi \in \mathscr{Q}^{s+1}, \Gamma\left(\varphi^{*} g\right)=\varphi^{*} \Gamma(g)$. Thus if $\varphi \in I_{r}^{s+1}$ and $g \in \mathscr{P}_{f}^{*}, \Gamma\left(\varphi^{*} g\right)=$ $\varphi^{*} \Gamma(g)=\varphi^{*} \Gamma=\Gamma$ so $\varphi^{*} g \in \mathscr{F}_{r}^{s}$. Thus $I_{r}^{s+1}$ acts by pullback on $\mathscr{F}_{r}^{s} A: I_{r}^{s+1} \times$ $\mathscr{F}_{r}^{s} \rightarrow \mathscr{F}_{r}^{s}$ and this action is continuous.

For $g \in \mathscr{A}^{s}$, let $I_{g}^{s+1}=\left\{\varphi \in \mathscr{D}^{s+1}: \varphi^{*} g=g\right\}$ denote the isometry group of $g$, and let $l_{g}^{\text {s+1 }}$ denote the connected component of the identity. Similarly, let $I_{r(s)}^{s+1}$ and $I_{r(s)}^{s+1}$ denote the affine group and connected component of the identity. Since $M$ is compact, by a result of Yano [34] (adapted to $H^{s}$ metrics), $I_{g}^{s+1}=I_{r_{(g)}}^{s+1}$ so in the above action $A, I_{r}^{s+1}$ is a common normal isotropy group for all $g \in \mathscr{F}{ }^{2}$ Thus $A$ is not an effective action, so we let $D$ be the discrete group $I_{r}^{s+1} / I_{r}^{s+1}$ an $\bar{A}: D \times \mathscr{F}_{r}^{j} \rightarrow \mathscr{F}_{r}^{s}$ be the associated effective action by $D$.

Note that if $g \in \mathscr{F}_{r}^{s}, \varphi \in I_{r}^{s+1}, \varphi \notin I_{g}^{s+1}$, then $\varphi^{*} g \in \mathscr{F}_{r}^{s}$ as above but $\varphi^{*} g \neq g$. Thus $\varphi^{*} g$ and $g$ are distinct isometric metrics in $\mathscr{F}_{r}^{*}$. Thus $\mathscr{F}_{r}^{3}$ intersects the orbit $O_{g}$ through $g$ once for each coset in $I_{\Gamma}^{s+1} / I_{g}^{s+1}$.

For $\Gamma \in \mathscr{H}^{s-1}$, the homogeneous space $\mathscr{D}^{s+1} / I_{r}^{s+1}$ can be given the structure of a $C^{\infty}$ manifold by using Theorem 1.3.1 and methods of Ebin [13].

1.3.3. TheOREM. Let $\Gamma \in \mathscr{H}^{s-1}, s>n / 2+1$. Then the space $\mathscr{H}^{p-1}$ of fiat riemannian $H^{s-1}$ connections is homeomorphic to the homogeneous space $\mathscr{D}^{s+1} / I_{\Gamma}^{s+1}$. Using the above action $\bar{A}$, the associated homogeneous fiber bundle is

$$
\pi: \mathscr{F}^{s} \rightarrow \mathscr{H}^{s-1} \approx \mathscr{D}^{s+1} / I_{r}^{s+1}
$$

where the projection $\pi(g)=\Gamma(g)$ is the Levi-Civita connection of $g$, and the fibers $\pi^{-1}(\Gamma)=\mathscr{F}_{r}$ are finite-dimensional manifolds. Thus $\mathscr{F}^{3}$ is the total space of a homogeneous fiber bundle, and, moreover, $F^{s}$ is a smooth closed submanifold of $\mathscr{A}^{s}$.

Proor. Since all $C^{\infty}$ flat riemannian connections on a compact connected manifold are affinely equivalent [35, Theorem 3.3.1], $\mathscr{D}$ acts transitively on $\mathscr{H}$ so that $\mathscr{H} \approx \mathscr{D} \mid I_{r}$ for $\Gamma \in \mathscr{H}$. From Theorem I.3.1, if $\Gamma_{1}, \Gamma_{2} \in \mathscr{H}^{s-1}$, there exist $\varphi_{1}, \varphi_{2} \in \mathscr{Q}^{s+1}$ such that $\varphi_{1}^{*} \Gamma_{1}, \varphi_{2}^{*} \Gamma_{2} \in \mathscr{H}$. Thus from the transitivity of $\mathscr{D}$ on $\mathscr{H}$, there exists $\psi$ $E \mathscr{D}$ such that $\psi^{*}\left(\varphi_{1}^{*} \Gamma_{1}\right)=\varphi_{2}^{*} \Gamma_{2}$ so that $\Gamma_{2}=\left(\varphi_{1} \circ \psi \circ \varphi_{2}^{-1}\right)^{*} \Gamma_{1}, \varphi_{1} \circ \psi \circ \varphi_{2}^{-1} \in \mathscr{D}^{s+1}$. Thus $\mathscr{D}^{s+1}$ acts transitively on $\mathscr{H}^{s-1}$. 
Let $\Psi_{x}$ be the linear holonomy group of $\Gamma$ at $x \in M$, a finite group [35, Corollary 3.4.7]. Then $\mathscr{F}_{r}$ is in one-to-one correspondence with the $\Psi_{x}$-invariant inner products on $T_{x} M$ (this is proved as in [35, Theorem 3.4.5]). By exponentiation, the $\Psi_{x}$-invariant inner products on $T_{x} M$ are diffeomorphic to the $\Psi_{x}$-invariant symmetric bilinear forms on $T_{x} M$, a finite-dimensional linear space so that $\mathscr{F}_{r}$ is a finite-dimensional manifold.

Let $\pi: \mathscr{F s} \rightarrow \mathscr{H}^{s-1}$ map each $g$ to its Levi-Civita connection $\Gamma(g)=\pi(g)$. Thus for $\Gamma \in \mathscr{H}^{s-1}, \pi^{-1}(I)=\mathscr{F}_{r}$. Using the action $\bar{A}: D \times \mathscr{F}_{r} \rightarrow \mathscr{F}_{r}^{3}$ on each fiber $F_{r}^{\prime}=\pi^{-1}(\Gamma), \pi: \mathscr{F}^{s} \rightarrow \mathscr{H}^{x-1}$ can be given the structure of an associated homogeneous fiber bundle over the homogeneous space $\mathscr{H}^{s-1} \approx \mathscr{D}^{s+1} / I_{\Gamma}^{s+1}$, where the "twisting" of the bundle is given by $\bar{A}$.

Using I.3.1 as before we get a $C^{\infty}$ structure for $\mathscr{F}^{3}$. The map injecting $\mathscr{F}^{3}$ to $\mathscr{M}^{s}$ can be seen to be a smooth immersion with closed range so $\mathscr{F}^{s}$ is a closed $C^{\infty}$ submanifold of $\mathscr{A}^{3}$. See [18] for details.

Remark. The set of isometry classes of flat riemannian metrics on $M$ is in bijective correspondence with $\mathscr{F}^{s} / \mathscr{D}^{s+1}=\mathscr{F}_{r}^{s} / I_{r}^{s+1}=\mathscr{F}_{r}^{3} / D$. Although $\mathscr{F}_{r}^{s}$ is a finitedimensional manifold, unfortunately the quotient space $\mathscr{F F}_{r}^{r} / D$ is not a manifold, as $\vec{A}: D \times \mathscr{F}_{r} \rightarrow \mathscr{F}_{r}^{\prime}$ does not act freely. In [36], Wolf describes the set $\mathscr{F}_{r}^{\prime} / D$ explicithy, as a double coset space. For example, if $\Gamma$ is a fiat riemannian connection on

$$
\begin{gathered}
M=T^{*}, \mathscr{F}_{r}=O(n) \backslash G L(n ; R), I_{r}=T^{n} \cdot G L(n, Z), \\
D=I_{r} / I_{r}=G L(n, Z) \text { and } \mathscr{F}_{r} / D=O(n) \mid G L(n ; R) / G L(n ; Z) .
\end{gathered}
$$

Note that $\mathscr{F}_{r}=O(n) \backslash G L(n ; R)$ is isomorphic to $\operatorname{Pos}(n ; R)$, the space of inner products on $R^{n}$, since the linear holonomy group of $T^{n}$ is the identity.

We can actually compute explicitly the tangent spaces of $\mathscr{F}^{s}$ and $\mathscr{F}_{r}^{3}$. First we remark that the splitting $S_{2}^{s}=\tilde{S}_{2}^{\xi} \oplus \alpha_{g}\left(\mathscr{X}^{s+1}\right), \dot{S}_{2}^{s}=$ ker $\delta_{g}$ for $g \in \mathscr{M}^{s+1}$ is valid for $g \in g^{s}$ as yas mentioned in I.1. and the proof of 1.2.3. For by 1.3.1, let $\varphi \in \mathscr{D}^{s+1}$ be such that $\bar{g}=\varphi^{*} g \in \mathscr{F}$. Let

$$
S_{2}^{s}=\operatorname{ker} \delta_{\bar{g}} \oplus \alpha_{\bar{g}}\left(\mathscr{X}^{s+1}\right)
$$

be the splitting of $S_{2}^{s}$ with respect to $\bar{g}$. This provides us with a splitting of $S_{2}^{s}$ with respect to $g$ as follows. Suppose $h \in S_{2}^{s}$. Then $\bar{h}=\varphi^{*} h \in S_{2}^{s}=\operatorname{ker} \delta_{\vec{z}} \oplus \alpha_{\vec{B}}\left(x^{s+1}\right)$; thus

$$
\varphi^{*} h=\bar{h}=\bar{h}^{\circ}+L_{X} \bar{g} \quad \text { where } \delta_{\bar{g}}\left(\bar{h}^{\circ}\right)=0 .
$$

Thus $\left(\varphi^{-1}\right)^{*} \bar{h}=h=\left(\varphi^{-1}\right)^{*} \bar{h}^{\mathrm{a}}+\left(\varphi^{-1}\right)^{*} L_{X} \overline{\bar{g}}=h+L_{(\varphi)_{0} \times} g$ where $h=\left(\varphi^{-1}\right)^{*}(\bar{h})^{\circ}$ $\in S_{2}^{s}$ since by pulling back $\delta_{\bar{g}}(\hat{h})=0,\left(\varphi^{-1}\right)^{*} \delta_{\bar{g}}(h)=\delta_{g}\left(\dot{\varphi}^{-1}\right)^{*}\left(\bar{h}^{\circ}\right)=0$. Note that even though the pushed forward vector field $(\varphi)_{*} X$ is only $H^{s}$ (so $L_{\varphi \cdot \times} g$ might be $\left.H^{s-1}\right), L_{p_{e}} X g=\left(\varphi^{-1}\right)^{*} L_{X} \bar{g}$ is actually $H^{s}$.

Now we compute the tangent spaces of $\mathscr{F}^{s}$ and $\mathscr{F}_{r}^{s}$.

I.3.4. THEOREM. For $g \in \mathscr{F}{ }^{3}, s>(n / \overline{2})+1$, let $\Gamma(g)=\Gamma$ denote the Levi-Civita connection of $g$. Then $T_{g} \mathscr{F}_{r}^{s}=\left\{h \in S_{2}^{J}: \nabla h=0\right\}=S_{2}^{\prime \prime}$, the parallel symmetric 2-tensors, and $T_{g} \mathscr{F}^{s}=\{h: \nabla h=0\}=S_{2}^{\prime \prime} \oplus \alpha_{g}\left(\mathscr{X}^{s+1}\right)$, where $h$ is the divergence free piece of $h$. 
Proor. To compute the tangent space of $\mathscr{F}_{R}^{s}$, we differentiate the condition $\Gamma(g(\lambda))=\Gamma$ for curves $g(\lambda) \in \mathscr{F}_{\Gamma}^{s}$. This yields

$$
\left.\frac{d}{d \lambda}(\Gamma(g(\lambda)))\right|_{2=0}=D \Gamma(g) \cdot h=0
$$

where $h=d g(0) / d \lambda \in S_{2}^{*}$. In local coordinates, this condition is

$$
\frac{1}{2}\left(h_{j \mid k}+h_{k \mid j}-h_{j k}^{1 j}\right)=0
$$

Taking the trace of this condition with respect to ik yields $d \operatorname{tr} h=0$, and with respect to ij yields $\delta h+\frac{1}{2} d \operatorname{tr} h=0$, and thus $\delta h=0$.

Taking another covariant derivative, contracting with $i$, commuting covariant derivatives (since $g$ is flat), and using $\delta h=0$, gives

$$
0=h_{j|k| i}+h_{k|j| i}-h_{j k}\left|i_{i i}=h_{j|i| k}+h_{k|i| j}-h_{j k}\right| i_{1 i}=-h_{j k} \mid i_{1 i}=\Delta h
$$

where $\lambda_{h}=-\nabla^{\cdot} \nabla_{i}^{\prime} h$ is the rough Laplacian. However on a compact manifold $\bar{\Delta} h=0$ implies that $\nabla h=0$ (since $0=\int h \cdot \not h d \mu_{g}=\int(\nabla h)^{2} d \mu_{g} \Rightarrow \nabla h=0$ ).

To find the tangent space of $T_{g} \mathscr{F}$, let $g(\lambda) \in \mathscr{F}^{s}$ be a smooth curve through $g$ with tangent $h$, and so in particular $\operatorname{Ric}(g(\lambda))=0$. Differentiating and using the variational equations for the Ricci tensor in [25] gives

$$
\begin{aligned}
0 & =\left.\frac{d}{d \lambda} \operatorname{Ric}(g(\lambda))\right|_{\lambda=0}=\left.D \operatorname{Ric}(g(\lambda)) \cdot \dot{h}(\lambda)\right|_{\lambda=0} \\
& =D \operatorname{Ric}(g) \cdot h=\frac{1}{2}\left(\Delta_{L} h-2 \delta^{*} \delta h-\text { Hess tr } h\right)
\end{aligned}
$$

where $\Delta_{L} h=\bar{L} h+R_{i h} h_{j}^{k}+R_{j k} h_{j}^{k}-2 R_{i}^{a} j h_{a b}$ is the Lichnerowicz Laplacian [25] acting on symmetric 2-tensors, and $\delta^{*}$ is the adjoint of $\delta$.

Let $h=h+L_{x g}$. Then

$$
\begin{aligned}
D \operatorname{Ric}(g) \cdot h & =D \operatorname{Ric}(g) \cdot\left(h+L_{X} g\right)=D \operatorname{Ric}(g) \cdot h+L_{X} \operatorname{Ric}(g) \\
& =D \operatorname{Ric}(g) \cdot h=\frac{1}{2}\left(\Delta_{L} h-H e s s \operatorname{tr} h\right)=0
\end{aligned}
$$

since $\operatorname{Ric}(g)=0$ and hence $L_{X} \operatorname{Ric}(g)=0$. To see that $D \operatorname{Ric}(g) \cdot L_{X} g=L_{X} \operatorname{Ric}(g)$, let $\psi_{t}$ be the flow of $X$ and differentiate the identity $\operatorname{Ric}\left(\psi_{\lambda}^{*} g\right)=\psi_{\lambda}^{*} \operatorname{Ric}(g)$ to get

$$
\left.\frac{d}{d \lambda} \operatorname{Ric}\left(\psi_{\lambda}^{*} g\right)\right|_{\lambda=0}=\left.D \operatorname{Ric}\left(\psi_{\lambda}^{*} g\right) \cdot \frac{d}{d \lambda}\left(\phi_{\lambda}^{*} g\right)\right|_{\lambda=0}=D \operatorname{Ric}(g) \cdot L_{X g}
$$

for the left-hand side and

$$
\frac{d}{d \lambda} \psi_{\lambda}^{*} \operatorname{Ric}(g)=L_{X} \operatorname{Ric}(g)
$$

for the right-hand side.

Taking the trace of $\Delta_{L} h-$ Hess $\operatorname{tr} h=0$ gives $\Delta \operatorname{tr} h=0$ (since $\operatorname{tr}\left(\Delta_{L} h\right)=$ $\Delta \operatorname{tr} h$ ) so that $\operatorname{tr} h=$ constant. Thus $\Delta_{L} h=0$ and since $g$ is flat, $\Delta_{L} h=\bar{\Delta} h$ and hence $\nabla h=0$. 
It is interesting that a variation of the Ricci equations around a flat metric is already enough to imply that $\nabla h=0$. This fact, discovered by Berger [2], is essentially the first order version of 1.3 .2 ; if $\mathscr{F}^{s} \neq \varnothing$, then $\mathscr{F}^{s}=\mathscr{E}_{0}^{s}$.

Note that $F^{s}$ is an invariant subset under the action of $\mathscr{D}^{s+1}$ (i.e., $\varphi^{*} g_{F} \in \mathscr{F}^{s}$ ), whereas $\mathscr{F}_{r}^{s}$ is not, since if $g \in \mathscr{F}_{\Gamma}^{3} \varphi^{*} g \in \mathscr{F}_{p \circ}^{s}$. That $T_{B} \mathscr{F}^{3}$ contains $\alpha_{\sigma}\left(\mathscr{Q}^{3}\right)$ whereas $T_{g} F_{r}$ does not is the infinitesimal version of this remark.

We also remark that since $T_{g} \mathscr{F}_{r}^{s}=S_{2}^{s+1} \subset \dot{S}_{2}^{z+1}, \mathscr{F}_{r}^{s}$ is, in a neighborhood of $g$, orthogonal in the $L_{2}$ metric on $\mathscr{M}^{3}$ (see [13]) to the orbit $\mathcal{O}_{g}$ through $g$. Nonetheless, we have seen (after 1.3.2) that $F_{r}^{3}$ bends back to intersect $\mathcal{O}_{g}$ at metrics $\varphi^{*} g \in \mathcal{O}_{g}$, $\varphi \in I_{r}^{s+1}, \varphi \notin I_{g}^{s+1}$.

In I.5 we will need the following result regarding the volume elements of flat metrics:

I.3.5. Theorem. Let $g, \bar{g} \in \mathscr{F}_{i s}^{s} s>n / 2+1$, and let $d \mu_{g}$ and $d \mu_{g}$ be the volume elements of $g$ and $\bar{g}$, respectively. Then there exists a constant $c>0$ such that $d \mu_{g}=c d \mu_{g}$

Proof. Let $\left(U, \psi_{U}\right)$ be a chart on $M$ such that the Christoffel symbols of $\Gamma=$ $\Gamma(g)=\Gamma(\bar{g})$ are $\Gamma_{j k}^{i}=0$. Thus $\partial g_{i j} / \partial x^{k}=0$ and $\partial \bar{g}_{i j} / \partial x^{k}=0$ on $U$ so that $(g \mid U)_{i j}$ $=c_{i j}$ and $(\bar{g} \mid U)_{i j}=\bar{c}_{i j}$ are matrices of constants. In this coordinate chart $d \mu_{g} \mid U$ $=\left(\operatorname{det} \bar{c}_{i j}\right)^{1 / 2} d x^{1} \wedge \cdots \wedge d x^{m}$ and $d \mu_{\dot{g}} \mid U=\left(\operatorname{det} \bar{c}_{i j}\right)^{1 / 2} d x^{1} \wedge \cdots \wedge d x^{n}$. so that $d \mu_{g} \mid U$ $=c_{U}\left(d \mu_{g} \mid U\right),=$ where $c_{U}=\left(\operatorname{det} \bar{c}_{i j}\right)^{1 / 2} /\left(\operatorname{det} \bar{c}_{i j}\right)^{1 / 2}$ is constant on $U$.

Now suppose $\left(V, \phi_{V}\right)$ is another coordinate chart for which $U \cap V \neq \varnothing$. Then $d \mu_{g} \mid V=c_{V}\left(d \mu_{g} \mid V\right)$ and on the intersection $U \cap V, d \mu_{g} \mid U \cap V=$ $c_{U}\left(d \mu_{g} \uparrow U \cap V\right)=c_{V}\left(d \mu_{\dot{\delta}} \mid U \cap V\right)$, so that $c_{U}=c_{V}$. Thus there exists a global $c=$ constant $>0$ such that $d \mu_{g}=c d \mu_{\bar{g}}$.

1.4. Linearization-instability around Ricci-flat metrics. If $g \in \mathscr{H}^{\mathrm{s}}$ is Ricci-fiat, then the map $R(\cdot) \rightarrow H^{s-2}$ is singular at $g$ in the sense that $D R(g): S_{2}^{s} \rightarrow H^{s-2}$ is not surjective. The failure of $D R(g)$ to be surjective suggests the equation $R(g)=0$ is linearization unstable in a neighborhood of a Ricci-flat solution for $\operatorname{dim} M \geqq 3$; i.e. there will be first order deformations $h \in S_{2}^{z}$ that will not be tangent to any curve $g(\lambda)$ of exact solutions of $R(g)=0$. In this section we analyze the structure of the map $\boldsymbol{R}(\cdot)$ at Ricci-flat (and fiat) metrics, and work out the extra condition that a first order deformation must satisfy in order that it be tangent to a curve of exact solutions of $R(g)=0$.

Our main computation is contained in the following: .

1.4.1. TheOREM. Let $g \in . \mathscr{K}^{s}, s>n / 2+1$, $\operatorname{Ric}(g)=0$. Then for $h \in T_{g} \mathbb{M}^{s}=S_{2}^{s}$,

$$
\int D^{2} R(g) \cdot(h, h) d \mu_{z}=-\frac{1}{2} \int\left(h \cdot \Delta_{L} h\right) d \mu_{g}-\frac{1}{2} \int(d \mathrm{tr} h)^{2} d \mu_{g}+\int(\delta h)^{2} d \mu_{g}
$$

where $\Delta L h=\Delta h-2 R_{i} p h_{a b}$ is the Lichnerowicz Laplacian [25] for a Ricci-flat space. If $g \in \mathscr{M}^{s+1}$ and $h=h+L_{X \bar{g}}, h \in \dot{S}_{2}^{s}, X \in X^{s+1}$ is the canonical decomposition of $h$, then 


$$
\begin{aligned}
\int D^{2} R(g) \cdot(h, h) d \mu_{g}= & -\frac{1}{2} \int h \cdot \Delta_{L} h d \mu_{g}-\frac{1}{2} \int(d \operatorname{tr} h)^{2} d \mu_{g} \\
& +2 \int(d \operatorname{tr} h) \cdot(d \delta X) d \mu_{\xi} .
\end{aligned}
$$

Proor.

$$
\begin{aligned}
\int D^{2} R(g) \cdot(h, h) d \mu_{g}= & D\left(\int D R(g) \cdot h d \mu_{g}\right) \cdot h-\int(D R(g) \cdot h)\left(D\left(d \mu_{g}\right) \cdot h\right) \\
= & D\left(\int(\Delta \mathrm{tr} h+\delta \delta h-h \cdot \operatorname{Rid}(g)) d \mu_{g}\right) \cdot h \\
& -\int(D R(g) \cdot h)\left(D\left(d \mu_{g}\right) \cdot h\right) \\
= & -D\left(\int h \cdot \operatorname{Ric}(g) d \mu_{g}\right) \cdot h-\int(D R(g) \cdot h)\left(D\left(d \mu_{g}\right) \cdot h\right)
\end{aligned}
$$

since $\int(\Delta \operatorname{tr} h+\delta \delta h) d \mu_{g} \equiv 0$ for all $(g, h)$ by Stokes' theorem. Since $\operatorname{Ric}(g)=0$, all contributions due to the metric terms in the pointwise contraction $h \cdot \operatorname{Ric}(g)$ $=g^{a b} g^{e d} h_{a c} R_{s d}$ are zero and so we have

$$
\begin{aligned}
\int D^{2} R(g) \cdot(h, h) d \mu_{z}= & -\int h \cdot(D \operatorname{Ric}(g) \cdot h) d \mu_{g}-\int(\Delta \operatorname{tr} h+\delta \delta h) \frac{1}{2} \operatorname{tr} h d \mu_{g} \\
= & -\frac{1}{2} \int h \cdot\left(\Delta L h-2 \delta^{*} \delta h-H e s s \text { tr } h\right) d \mu_{g} \\
& -\frac{1}{2} \int(\Delta \operatorname{tr} h+\delta \delta h) \operatorname{tr} h d \mu_{g} \\
= & -\frac{1}{2} \int h \cdot \Delta L h d \mu_{g}+\int(\delta h)^{2} d \mu_{g}+\frac{1}{2} \int \delta h \cdot d \operatorname{tr} h d \mu_{z} \\
& -\frac{1}{2} \int(d \operatorname{tr} h)^{2} d \mu_{g}-\frac{1}{2} \int \delta h \cdot d \operatorname{tr} h d \mu_{z} \\
= & -\frac{1}{2} \int h \cdot \Delta L h d \mu_{g}-\frac{1}{2} \int(d \operatorname{tr} h)^{2} d \mu_{g}+\int(\delta h)^{2} d \mu_{g}
\end{aligned}
$$

where we have used $D\left(d \mu_{g}\right) \cdot h=\frac{1}{2}(\operatorname{tr} h) d \mu_{2}$ and have integrated several times by parts.

Now suppose $g \in \mathscr{M}^{s+1}, \operatorname{Ric}(g)=0$, and $h=h+L_{X g}$. Then from the proof of I.3.4, $D \operatorname{Ric}(g) \cdot h=D \operatorname{Ric}(g) \cdot h=\frac{1}{2}\left(\Delta_{L} h-\right.$ Hess tr $\left.h\right)$, and similiarly $D R(g) \cdot h$ $=D R(g) \cdot h=\Delta \mathrm{tr} h$. Thus from (1) above,

$$
\begin{aligned}
\int D^{2} R(g) \cdot(h, h) d \mu_{g} & =-\frac{1}{2} \int h \cdot(\Delta h \hat{h}-\text { Hess tr } h) d \mu_{g}-\frac{1}{2} \int(\Delta \operatorname{tr} h)(\operatorname{tr} h) d \mu_{g} \\
& =-\frac{1}{2} \int h \cdot \Delta_{L} h d \mu_{g}+\frac{1}{2} \int(\delta h-d \operatorname{tr} h) \cdot d \operatorname{tr} h d \mu_{g}
\end{aligned}
$$

where we have used the fact that for Einstein spaces $\delta \circ \Delta_{L}=\Delta \circ \delta$ (see [25]), so that $\Delta_{L} h \in \xi_{2}^{s-2}$, and so by orthogonality of $\xi_{2}^{s-2}$ and $\alpha_{g}\left(\mathscr{X}^{s+1}\right), \int h \cdot \Delta_{L} h d \mu_{g}=$ $\int h \cdot \Delta_{L} h d \mu_{g}$.

Since $h=h+L_{x g}, \delta h=\delta L_{x g}=\Delta X+(d \delta X)^{*}=(2 d \delta X+\delta d X)^{z}$ and $\operatorname{tr} h=$ tr $h-2 \delta X$. Thus $(\delta h)^{2}-d \operatorname{tr} h=4 d \delta X+\delta d X b-d$ tr $h$ and 


$$
\begin{aligned}
\frac{1}{2} \int\left((\delta h)^{2}-d \operatorname{tr} h\right) \cdot d \operatorname{tr} \stackrel{h}{h} d \mu_{g} & =\frac{1}{2} \int\left(4 d \delta X+\delta d X^{b}-d \operatorname{tr} \grave{h}\right) \cdot d \operatorname{tr} \grave{h} d \mu_{g} \\
& =-\frac{1}{2} \int(d \operatorname{tr} \grave{h})^{2} d \mu_{g}+2 \int d \delta X \cdot d \operatorname{tr} \grave{h} d \mu_{g}
\end{aligned}
$$

since $\delta d X^{b}$ and $d$ tr $h$ are $L_{2}$-orthogonal.

The extra condition that a first order deformation of $R(g)=0$ about a Ricciflat solution must satisfy for it to be tangent to a curve of exact solutions is now easily computed.

I.4.2. ThEOREM. Let $g(\lambda) \in \mathscr{M}^{3}, \lambda \in(-\delta, \delta), \delta>0, s>n / 2+1$ be a $C^{2}$ curve with $\left(g(0), g^{\prime}(0)\right)=(g, h)$. Suppose $g \in \mathscr{A}^{s+1}$, and let $h=h+L_{x g}$ be the canonical decomposition of $h$. If $R(g(\lambda))=0$ and $\operatorname{Ric}(g)=0$, then $\operatorname{tr} h=$ constant and

$$
\int h \cdot \Delta_{L} h d \mu_{g}=0 \text {. }
$$

If $g \in \mathscr{F}$, then $\nabla h=0$.

Proor. Differentiating $R(g(\lambda))=0$ twice and evaluating at $\lambda=0$ gives

$$
\begin{aligned}
\left.\frac{d}{d \lambda} R(g(\lambda))\right|_{\lambda=0} & =\left.D R(g(\lambda)) \cdot \frac{d g}{d \lambda}(\lambda)\right|_{\lambda=0}=D R(g) \cdot h \\
& =\Delta \operatorname{tr} h+\delta \delta h=\Delta \operatorname{tr} h=0,
\end{aligned}
$$

(2) $\left.\frac{d^{2} R}{d \lambda^{2}}(g(\lambda))\right|_{\lambda=0}=\left.D^{2} R(g(\lambda)) \cdot\left(\frac{d g}{d \lambda}(\lambda), \frac{d g}{d \lambda}(\lambda)\right)\right|_{2=0}+\left.D R(g(\lambda)) \cdot\left(\frac{d^{2} g}{d \lambda^{2}}\right)(\lambda)\right|_{\lambda=0}$ $=D^{2} R(g) \cdot(h, h)+D R(g) \cdot g^{\prime \prime}(0)=0$.

Here we are identifying $T \mathscr{M}^{s}$ with $\mathscr{M}^{s} \times S_{2}^{z}$, so $d g(\lambda) / d \lambda \in S_{2}^{s}$ and $d^{2} g(\lambda) / d \lambda^{2} \in S_{2}^{s}$.

Integrating (2) over $M$ (using the volume element $d \mu_{g}$ ) gives the extra condition

$$
\int D^{2} R(g) \cdot(h, h) d \mu_{z}=0
$$

since $\int D R(g) \cdot g^{\prime \prime}(0) d \mu_{g}=\int\left(\Delta \operatorname{tr}\left(g^{\prime \prime}(0)\right)+\delta \delta\left(g^{\prime \prime}(0)\right)\right) d \mu_{B}=0$ for all accelerations $g^{\prime \prime}(0)$.

From (1), tr $h=$ constant, so that from I.4.1, (3) becomes

$$
\begin{aligned}
-\frac{1}{2} \cdot \int h \cdot \Delta_{L} h d \mu_{g}-\frac{1}{2} \int(d \operatorname{tr} h)^{2} \cdot d \mu_{g} & +2 \int d \operatorname{tr} h \cdot d \delta X^{b} d \mu_{g} \\
& =-\frac{1}{2} \int h \cdot \Delta_{\nu} h d \mu_{g}=0 .
\end{aligned}
$$

If $g$ is flat, $\Delta_{L}=\bar{\Delta}$, so that $0=\int h \cdot \bar{J} h d \mu_{g}=\int h \cdot \Delta h d \mu_{g}=\int(\nabla h)^{2} d \mu_{g} \Rightarrow \nabla h$ $=0$.

Remarks. 1. At a regular point $g \in \mathscr{H}_{0}^{3}$ where $D R(g)$ is surjective (so that $\operatorname{Ric}(g) \neq 0$ ), equation (2), when integrated over $M$, gives

$$
\int D^{2} R(g) \cdot(h, h) d \mu_{g}-\int \operatorname{Ric}(g) \cdot g^{\prime \prime}(0) d \mu_{g}=0,
$$


an integrated condition on $g^{\prime \prime}(0)$ in terms of $h$, which at regular points $g$ does not give an extra condition on $h$. It is only when $\operatorname{Ric}(g)=0$ that the term involving $g^{\prime \prime}(0)$ drops out leaving an integrated extra condition on $h$.

2. That we get one extra condition on $h$ when $\operatorname{Ric}(g)=0$ corresponds to the fact that $\operatorname{ker}(D R(g))^{*}=$ (constant functions on $M$ \} is 1-dimensional, since as in $\$ 0$, Example 4, Remark 2, there is an extra condition for each dimension in $\operatorname{ker}(D R(g))^{*}$. In the case at hand, the equation $\int D R(g) \cdot g^{\prime \prime}(0) d \mu_{g}=0$ (which leads to the extra condition on $h$ ) can be expressed as

$$
\int\left(D R(g)^{*} 1\right) \cdot g^{\prime \prime}(0) d \mu_{g}=0 \text { for all } g^{\alpha}(0) \text { iff } 1 \in \operatorname{ker}(D R(g))^{*} \text {. }
$$

That the extra condition on $h$ is an integrated condition corresponds to the fact that $(D R(g))^{*}$ is an $L_{Z}$ adjoint; i.e. we do not get an extra condition on $h$ until we integrate (2) against an element of ker $(D R(g))^{*}$; cf. Example 4, Remark 1.

3. If $g \in \mathscr{M}^{s}, \operatorname{Ric}(g)=0$, but $g$ is not of class $H^{s+1}, h$ may not have a canonical decomposition. In this case, by using the first order condition $D R(g) \cdot h=\Delta \operatorname{tr} h$ $+\delta \delta h=0$ and equation (1) in the proof of I.4.1, the extra condition (3) can be expressed as

$$
\begin{aligned}
\int D^{2} R(g) \cdot(h, h) d \mu_{g}= & -\int h \cdot(D \operatorname{Ric}(g) \cdot h) d \mu_{g} \\
= & -\frac{1}{2} \int h \cdot\left(\Delta_{L} h-2 \delta^{*} \delta h-H e s s \operatorname{tr} h\right) d \mu_{g} \\
= & -\frac{1}{2} \int h \cdot \Delta_{L} h d \mu_{g}+\frac{1}{2} \int(\delta h)^{2} d \mu_{g} \\
& +\frac{1}{2} \int \delta h \cdot d \operatorname{tr} h d \mu_{g}=0 .
\end{aligned}
$$

4. Considering third and higher order derivatives of $R(g(\lambda))=0$, $\operatorname{Ric}(g(0))=0$, does not lead to any extra condition on the first order deformations. For example, differentiating

$$
D^{2} R(g(\lambda)) \cdot(h(\lambda), h(\lambda))+D R(g(\lambda)) \cdot g^{\prime \prime}(\lambda)=0
$$

(where $h(\lambda)=g^{\prime}(\lambda)$ ) and evaluating at $\lambda=0$ gives

$$
D^{3} R(g) \cdot(h, h, h)+3 D^{2} R(g) \cdot\left(h, g^{\prime \prime}(0)\right)+D R(g) \cdot g^{n}(0)=0 .
$$

Integrating over $M$, the last term again drops out, leaving

$$
\int D^{3} R(g) \cdot\left(h, h_{0} h\right) d \mu_{z}+3 \int D^{2} R(g) \cdot\left(h_{0} g^{\prime \prime}(0)\right) d \mu_{g}=0
$$

as the extra integrated "third order" condition on $g^{\prime \prime}(0)$ (beyond that implied by the second order pointwise condition of equation (2)) that has to be satisfied for $g^{\prime \prime}(0)$ to be the acceleration of some curve of exact solutions of $R(g)=0$. This of course is the analog of the second order phenomenon. This situation repeats, and in general there is an extra integrated condition on the $n$th order deformation that comes from the $(n+1)$ st order equations. However, these higher order equations do not provide any further conditions on the first order deformations in general. 
In special cases, however, it is possible that third order variations can lead to extra conditions on first order deformations, as in \$0, Example 3.

5. We can also examine the linearization instability of the equation $R(g)=\rho$ $=$ constant $>0$ around a solution $\left(S^{n}, g_{0}\right)$, a standard sphere in $R^{n+1}$ of radius $r_{0}=(n(n-1) / \rho)^{1 / 2}$. In this case, from Remark 2 of I.2.1, $\operatorname{ker}\left(D R\left(g_{0}\right)\right)^{*}=$ égenfunctions of $\left.\Delta_{g}\right\}$ so that if $f \in \operatorname{ker}\left(D R\left(g_{0}\right)\right)^{*}$,

$$
\int f D R\left(g_{0}\right) \cdot g^{\prime \prime}(0) d \mu_{g}=\int\left(D R\left(g_{0}\right)^{*} f\right) \cdot g^{\prime \prime}(0) d \mu_{g}=0
$$

for all $g^{\prime \prime}(0)$. Thus multiplying (2) by $f$ and integrating over $M$ gives for each linearly independent eigenfunction $f$ of $\Delta_{g_{0}}$ the extra condition

$$
\int f D^{2} R\left(g_{0}\right) \cdot(h, h) d \mu_{g_{0}}=0
$$

on a first order deformation $h$. That this really is extra is shown in [18].

In the case that $g_{r} \in \mathscr{F}^{\prime}$, the integrated extra condition $\int h \cdot \bar{J} h d \mu_{g,}=0$ can be converted to the very strong pointwise condition $\nabla h=0$. This pointwise condition signals an even greater type of instability, viz., that the flat solutions of $\boldsymbol{R}(g)=\mathbf{0}$ are isolated among all the solutions. This aspect of the map $R(\cdot)$ will be examined in the next section.

In the flat space case, if the extra condition $\nabla h=0$ is satisfied, then we can explicitly integrate up any deformation $h=h_{1}+L_{X} g_{F}, \nabla h_{1}=0$. Indeed, let $g_{F}^{-1} \cdot h_{1}$ denote the 1-contravariant 1-covariant form of $h_{1}$, let $\exp \left(g_{F}^{-1} \cdot h_{1}\right)$ denote the pointwise exponentiation of $g_{F}^{-1} \cdot h_{1}$, another tensor of type $(l)$, and let $g(\lambda)$ $=g_{F} \exp \left(\lambda g_{F}^{-1} \cdot h_{1}\right)$ denote the 2-contravariant form. In coordinates, $g_{F} \exp \left(\lambda g_{F}^{-1} \cdot h_{1}\right)$ $\left.=\left(g_{F}\right)_{i \mathrm{k}} \exp \left(\lambda_{g_{F}}\right)^{k}\left(h_{1}\right)_{l i}\right)$. Then $g(\lambda)$ is a $C^{\infty}$ curve in $\mathscr{F}_{\Gamma\left(g_{j}\right)}^{*}$, defined for all $\lambda \in R$, such that $\left(g(0), g^{\prime}(0)\right)=\left(g F, h_{1}\right)$. If $\varphi_{\lambda} \in \mathscr{D}, \varphi_{0}=$ id ${ }_{M}$, is the flow of the vector field $X$, then $\bar{g}(\lambda)=\varphi_{\lambda}^{*}(g(\lambda)) \in F^{3}, \vec{g}(0)=g_{F}$, and $\ddot{g}^{\prime}(0)=\varphi_{\lambda}^{*}\left(g^{\prime}(\lambda)+\left.L_{X} g(\lambda)\right|_{\lambda=0}=\right.$ $h_{1}+L_{X g F}$.

In the next section we shall see that $g(\lambda) \in \mathscr{F}^{\prime}$ is quite necessary if $g(\lambda) \in \mathscr{M}_{0}^{s}$ and $g(0)$ is flat.

If $\mathscr{F}^{\prime}=\varnothing$, and if there exist nonflat Ricci-flat metrics $g_{0}$ (so that $\operatorname{dim} M \geqq 4$ ), then we do not know if satisfaction of the extra condition $\int h \cdot \Delta_{L} h d \mu_{g_{0}}=0$ is sufficient to find a curve $g(\lambda) \in \mathscr{A l}_{0}^{s}, g(0)=g_{0}, g^{\prime}(0)=h$. However, because third and higher order deformations of $R(g)=0$ do not lead to any new conditions on $h$, we suspect that if the second order condition on $h$ is satisfied, then there is a curve $g(\lambda) \in \mathscr{H}_{0}^{s}$ which is tangent to $h$.

One of the difficulties here is that the structure of the set $\mathscr{E}_{0}^{s}=\left\{g \in \mathscr{M}^{s}\right.$ : $\operatorname{Ric}(g)=0\}$, if not empty, is unknown. In particular, we do not know if it is a manifold. The formal tangent space of $\mathscr{E}_{0}^{s}$ at $g \in \mathscr{H}^{\mathrm{st}}$ is, from the proof of 1.3 .4 , given by $\operatorname{ker}(D \operatorname{Ric}(g))=\left\{h \in S_{2}^{s}: \Delta_{L} h=0\right\}=\left\{\right.$ harmonic tensors of $\left.\Delta_{L}\right\}$. Thus if $\mathscr{E}_{0}^{s}$ were a mauifold, any $h$ such that $\Delta_{L} h=0$ is tangent to a curve in $\mathscr{E}_{0}^{s}$. This would partially answer the question of whether the extra condition of I.4.2 on a deformation $h$ is sufficient to find a curve in $A_{0}^{s}$ tangent to $h$. 
We now give an example of the linearization instability of the equation $R(g)=0$ on a flat 3-torus. Let $A$ be the lattice generated by the standard basis $\left\{\hat{e}_{i}\right\}$ in $R^{3}$, let $T^{3}=R^{3} / \Lambda$, and let $g_{F}$ be the metric induced on $T^{3}$ from $R^{3}$. Let $S^{1}$ be the circle with unit circumference, and let $f: S^{1} \rightarrow R$ be any smooth function, $f \neq$ constant. Set

$$
h=h_{i j}=\left(\begin{array}{ccc}
0 & f\left(x_{3}\right) & 0 \\
f\left(x_{3}\right) & 0 & 0 \\
0 & 0 & 0
\end{array}\right)
$$

Then $\delta h=0$ and tr $h=0$ (taken with respect to $g_{F}$ ), but $\nabla h \neq 0$. Thus this $h$, although a solution to the linearized equations $D R\left(g_{F}\right) \cdot h=\Delta t r h+\delta \delta h=0$, is not tangent to any curve $g(\lambda) \in \mathscr{H}_{0}^{3}, g(0)=g_{F}$, since $\nabla h \neq 0$.

If $f\left(x_{3}\right)=$ constant, then the extra condition $\nabla h=0$ is satisfied, and we can integrate $h$ up to a curve

$$
g(\lambda)=g_{F} \exp \left(\lambda g_{F}^{-1} \cdot h\right)=\left(\begin{array}{ccc}
\cosh \lambda f & \sinh \lambda f & 0 \\
\sinh \lambda f & \cosh \lambda f & 0 \\
0 & 0 & 1
\end{array}\right)
$$

of flat metrics on $T^{3}$ with $\left(g(0), g^{\prime}(0)\right)=\left(g_{F}, h\right)$.

REMARKs. 1. The above expression for $g(\lambda)$ is also valid if $f \neq$ constant, but the. $R(g(\lambda))$ need not be zero for $\lambda \neq 0$.

2. On a flat 2-torus $T^{2}, \delta h=0$ and $\operatorname{tr} h=$ constant imply $\nabla h=0$ so that we cannot construct our example. This corresponds to the fact that $R(g)=0$ is linearization stable if $\operatorname{dim} M=2$.

1.5. Isolated solutions of $R(g)=0$. In the flat space case, the emergence of a pointwise condition from the integrated extra condition signals that the flat solutions of $R(g)=0$ may be isolated solutions. In this section we show that this expectation is correct. We then use this result to work out the structure of $\mathscr{H}_{0}$ in the case that $\mathscr{F} \neq \varnothing$.

That $R(g)=0$ implies that $g$ is flat if $g$ is in a neighborhood of a flat metric is somewhat surprising in view of the fact that the scalar curvature is a relatively weak measure of the curvature.

Fix an $H^{\text {s }}$ volume element $d \mu$ on $M$, and define

$$
\Psi: \mathscr{M}^{s} \rightarrow R, \quad g \mapsto \int R(g) d \mu
$$

Note that $\Psi$ is not the usual integrated scalar curvature (cf. II.2), since in general $d \mu \neq d \mu_{g}$

1.5.1. THEOREM. A metric $g \in \mathscr{L}^{3}$ is a critical point of $\Psi$ if and only if $\operatorname{Ric}(g)=$ 0 and $d \mu=c d \mu_{g}$ for some constant $c>0$. At a critical metric $g_{c} \in \mathscr{M}^{s}$, the Hessian $d^{2} \Psi\left(g_{c}\right): S_{2}^{s} \times S_{2}^{s} \rightarrow R$ of $\varphi$ is given by 
$d^{2} \Psi\left(g_{\epsilon}\right) \cdot(h, h)=-\frac{1}{2} \int h \cdot \Delta_{L} h c d \mu_{8_{*}}-\frac{1}{2} \int(d \operatorname{tr} h)^{2} c d \mu_{s_{*}}+\int(\delta h)^{2} c d \mu_{8_{*}}$

Proop. For $g \in \mathscr{A}^{\mathrm{s}}$, let $p \in H^{\mathcal{s}}(M ; R), p>0$ be such that $d_{\mu}=p d \mu_{k}$. Then

$$
\begin{aligned}
d \Phi(g) \cdot h & =\int D R(g) \cdot h d \mu=\int(\Delta \operatorname{tr} h+\delta \delta h-h \cdot \operatorname{Ric}(g)) p d \mu_{g} \\
& =\int(g \Delta p+\operatorname{Hess} p-p \operatorname{Ric}(g)) \cdot h d \mu_{z}=0
\end{aligned}
$$

for all $h \in S_{2}$ iff

$$
\tau_{B}^{*}(p)=g \Delta p+\text { Hess } p-p \operatorname{Ric}(g)=0, \quad p>0 .
$$

From the proof of $1.2 .1,(1) \Rightarrow R(g)=$ constant $\geqq 0$. Contracting (l) gives

$$
(n-1) \Delta p=R(g) p, \quad p>0 ;
$$

integrating (2) over $M$ gives

$$
0=(n-1) \int \Delta p d \mu_{g}=R(g) \int p d \mu_{g}
$$

If $R(g)=$ constant $>0$, then $\int p d \mu_{g}=0$, contradicting $p>0$. Thus $R(g)=0$, so from (2), $p=$ constant $=c>0$, so that $d \mu=c d \mu_{g}$. Since $p=$ constant $>0$, from (1), $\operatorname{Ric}(g)=0$.

Now suppose $g_{c}$ is a critical metric of $\psi$, so $\operatorname{Ric}(g)=0, d_{\mu}=c d \mu_{\varepsilon_{0}}$ Thus

$$
d^{2} \Psi\left(g_{c}\right) \cdot(h, h)=\int D^{2} R\left(g_{c}\right) \cdot(h, h) d \mu=c \int D^{2} R\left(g_{c}\right) \cdot(h, h) d \mu_{g^{*}}
$$

The theorem now follows from 1.4.1.

RaMarks. 1. In the above it is important that we hold the volume element fixed and then let $d \mu=c d \mu_{g}$ after we take the derivatives; see 1.5.2, Remark 2 .

2. That $d \mu=c d_{\mu_{g}}^{t}$ at a critical point of $\Psi$ allows us to compute $d^{2} \Psi\left(g_{c}\right)$ from 1.4.1. Otherwise, the computation of $d^{2} \Psi(g) \cdot(h, h)=\int D^{2} R(g) \cdot(h, h) d \mu$ is considerably more complicated than the computation of $\int D^{2} R(g) \cdot(h, h) d \mu_{g}$ (unless $\left.d \mu=c d \mu_{g}\right)$, since $\int(\Delta \operatorname{tr} h+\delta \delta h) d \mu$ does not vanish for all $(\mathrm{g}, h)$ as in the proof of I.4.1.

3. W need not have any critical points; e.g. if $d \mu$ is chosen so as not to be the volume element of any Ricci-flat metric.

To second order, Brill [5] shows that the flat solutions of $R(g)=0$ are isolated, and Kazdan and Wamer [21, \$5] show that near a flat metric to second order there are no metrics with positive scalar curvature. The following extends these results to a full neighborhood of the flat metrics.

1.5.2. TheOREM. Let $g_{F} \in \mathscr{F}^{3}, s>n / 2+1$. Then there exists a neighborhood $U_{g r} \subset \mathscr{H}^{*}$ of $g_{F}$ such that if $g \in U_{g r}$ and $R(g) \geqq 0$, then $g$ is also in $\mathscr{F}^{\text {s. }}$.

Proof. Let $d \mu_{g}$, denote the volume element of $g_{F}$, and let $\Gamma$ denote its LeviCivita connection. Let $\Psi: \mathscr{A l}^{3} \rightarrow R, \Psi(g)=\int R(g) d \mu_{g r}$

If $\tilde{\mathrm{g}}_{F} \in \mathscr{F}_{F}^{s}$, by I.3.5, $d \mu_{\overline{\mathrm{g}},}=c d \mu_{g_{n},} c=$ constant $>0$, so that $\mathscr{F}_{\Gamma}^{s}$ is a critical 
submanifold of $\Psi$ (but not the entire critical submanifold), and at a critical point $\overline{\boldsymbol{g}}_{\boldsymbol{F}} \in \mathscr{F}_{F}^{3}$

$$
d^{2} \Psi\left(\tilde{g}_{F}\right) \cdot(h, h)=-\frac{1}{2} \int(\nabla h)^{2} c d \mu_{B_{g}}-\frac{1}{2} \int(d \operatorname{tr} h)^{2} c d \mu_{g,}+\int(8 h)^{2} c d \mu_{g_{r}}
$$

Let $S_{g_{r}}^{s}$ be a slice at $g_{F}$ (see the remark preceding Theorem I.3.4) and let $\Phi_{S}=$ $\Psi\left\lceil S_{g r}^{s}\right.$ Since $g_{F}$ is a critical point for $\Psi, g_{F}$ is also a critical point for $\Psi_{S}$ and the Hessian of $\Psi_{S}$ is given by restricting the Hessian of $\Psi$; thus from I.5.1., for $h \in$ $T_{g r} S_{g r}^{s}=\left\{h \in S_{2}^{3}: \delta_{g r} h=0\right\}$,

$$
d^{2} \Psi_{S}\left(g_{F}\right) \cdot(h, h)=d^{2} \Psi\left(g_{F}\right) \cdot(h, h)=-\frac{1}{2} \int(\nabla h)^{2} d \mu_{g r}-\frac{1}{2} \int(d \operatorname{tr} h)^{2} d \mu_{g r}
$$

Thus $d^{2} \Psi_{S}\left(g_{F}\right)$ is negative-definite on a complement to $T_{g} \mathscr{F}_{r}^{s}=\left\{h \in S_{2}^{s}: \nabla h=0\right\}$ in $T_{g r} S_{g r}^{g}$. Thus since $\mathscr{F}_{r}^{\prime}$ is critical there exists a neighborhood $V \subset S_{g_{r}}^{s}$ of $g F$ such that $\Psi_{S} \leqq 0$ on $V$, and if $\Psi_{S}(g)=0$, then $g \in \mathscr{F}_{r}$. Thus the critical submanifold $F_{r}^{3}$ is isolated among the zeros of $\Psi_{S}$.

Let $U_{g s}=\mathscr{P}^{s+1}(V)=\left\{\phi^{*} g \in \mathscr{A}^{s}: \varphi \in \mathscr{D}^{s+1}, g \in V\right\}$ be the saturation of $V$. By the slice theorem [13], $U_{g}$ fills out a neighborhood of $g_{F}$. Thus if $g \in U_{g,}$ and $R(g) \geqq 0$, there exists a $\varphi \in \mathscr{D}^{s+1}$ such that $\varphi^{*} g \in V \subset S_{g}^{s}$, and thus $\Psi_{S}\left(\varphi^{*} g\right) \leqq 0$, since $\Phi_{S}$ is negative on $V$. But $R\left(\varphi^{*} g\right)=R(g) \circ \varphi \geqq 0$ so that $\Psi_{S}\left(\varphi^{*} g\right)=\int R\left(\varphi^{*} g\right) d \mu_{R}$ $=\int R(g) \circ \varphi d \mu_{g} \geqq 0$. Thus $\Psi_{s}\left(\varphi^{*} g\right)=0$ so that $\varphi^{*} g \in \mathscr{F}_{r}^{*}$ and $g \in\left(\varphi^{-1}\right)^{*} \mathscr{F}_{r}^{3}$ $\subset \mathscr{F}^{3}$ is fiat.

Remarks. 1. For $\operatorname{dim} M=2$, we need not restrict to a neighborhood $U_{g r}$. Indeed, from the Gauss-Bonnet theorem, if $\mathscr{F s} \neq \varnothing$, the Euler-Poincare characteristic $\chi_{M}=0$, so that for any metric $g, \int R(g) d \mu_{g}=0$. Thus $R(g) \geqq 0 \Rightarrow R(g)=$ $0 \Rightarrow g$ is fiat.

2. Usually one considers an integrated scalar curvature $\Phi(g)=\int R(g) d \mu_{g}$ with volume element $d \mu_{g}$ induced from $g$ rather than $\Psi(g)=\int R(g) d \mu$ with fixed volume element $d \mu$, as e.g. in [3]. One then has (see II.2.2) at a critical flat metric

$$
d^{2} \Phi\left(g_{F}\right) \cdot(h, h)=-\frac{1}{2} \int(\nabla h)^{2} d \mu_{8,}+\frac{1}{2} \int(d \operatorname{tr} h)^{2} d \mu_{8,}
$$

rather than

$$
d^{2} \Psi\left(g_{F}\right) \cdot(h, h)=-\frac{1}{2} \int(\nabla h)^{2} d \mu_{g r}-\frac{1}{2} \int(d \operatorname{tr} h)^{2} d \mu_{g r} .
$$

There is now an important sign change in the second term; see also II.2.2, Remark 2. Brill [5] uses (1), together with the condition tr $h=$ constant implied by the first order equations $D R(g) \cdot h=\Delta \operatorname{tr} h=0$ to deduce $\nabla h=0$ and hence his second order result.

To extend this result to a full neighborhood of the flat metrics, one may not use the first order condition tr $h=$ constant. The indefinite sign in (1) now becomes 
a severe difficulty. We introduced the map $\Psi$ with fixed volume element $d \mu$ since this difficulty is not present in (2).

Note that if the first order condition tr $h=$ constant is used, $d^{2} \Psi\left(g_{F}\right) \cdot(h, h)=$ $d^{2} \phi\left(g_{F}\right) \cdot(h, h)=-\frac{1}{2} \int(\nabla h)^{2} d \mu_{g_{e}}$ so that the two treatments are then equivalent.

As a consequence of I.5.1, we have the following structure theorem for $\mathscr{H}_{0}^{s}$ :

1.5.3. Theorem. Let $s>n / 2+1, \operatorname{dim} M \geqq 3$, and if $\operatorname{dim} M \geqq 4$, assume Fs $\neq \varnothing$. Then $\mathscr{M}_{0}^{s}=\left(\mathscr{M}_{0}^{s}-\mathscr{F}^{s}\right) \cup \mathscr{F}^{s}$ is the disjoint union of $C^{\infty}$ closed submanifolds, and hence $\mathscr{M}_{0}^{3}$ is itself a $C^{\infty}$ closed submanifold of $\mathscr{A}^{\mathrm{s}} ;$ similarly, $\mathscr{M}_{0}=\left(\mathscr{H}_{0}\right.$ - F) $\cup \mathscr{F}$ is a $C^{\infty}$ closed submanifold of $\mathscr{M}$.

Notes. 1 . If $\operatorname{dim} M=2, \mathscr{H}_{0}^{s}=\mathscr{F}$ is also a $C^{\infty}$ closed submanifold.

2. We are allowing the possibility that $\mathscr{M}_{0}^{s}-\mathscr{F}^{s}$ is empty, and if $\operatorname{dim} M=3$, we are also allowing the possibility that $\mathscr{F}^{3}$ is empty.

3. $\mathscr{H}_{0}^{s}$ is a manifold since we are allowing different components of a manifold to be modelled on different Hilbert spaces.

Proof. If $\operatorname{dim} M=3, \operatorname{Ric}(g)=0 \Rightarrow g$ is fiat. If $\operatorname{dim} M \geqq 4$ and $g=\varnothing$, then from 1.3.2 $=\mathscr{E}_{0}^{3}$. Thus in either case

$$
\mathscr{M}_{0}^{s}=\left(\mathscr{H}_{0}^{s}-\mathscr{E}_{0}^{s}\right) \cup \mathscr{E}_{0}^{s}=\left(\mathscr{M}_{0}^{s}-\mathscr{F}^{s}\right) \cup \mathscr{F}^{s}
$$

which from 1.2.2 and I.3.2 is the disjoint union of $C^{\infty}$ submanifolds, $\mathscr{F}^{s}$ closed.

Let $g_{n} \rightarrow g$ be a convergent sequence in $\mathscr{H}_{0}^{s}-\mathscr{F}^{3}$. Then $g \in \mathscr{M}_{0}^{s}$, and if $g \in \mathscr{F}^{s}$, there exists a neighborhood $U_{g} \subset \mathscr{H}^{s}$ such that $\bar{g} \in U_{g}, R(g)=0 \Rightarrow g \in \mathscr{F}^{s}$. But then for $n$ sufficiently large, $g_{n} \in U_{g}, R\left(g_{n}\right)=0 \Rightarrow g_{n} \in \mathscr{F}^{3}$ contradicting $g_{n} \in \mathscr{A}_{0}^{s}$ - Fss. Thus $g \in \mathscr{H}_{0}^{s}-\mathscr{F} s$, a closed set.

Remarks. 1. There are various topological conditions on $M, \operatorname{dim} M \geqq 4$, that imply a Ricci-fiat metric is flat; see [19], [20]. If we adopt any of these conditions, then we can drop the $\mathscr{F} \neq \varnothing$ assumption. Of course, if it turns out that Ricciflat implies flat, then this assumption can be dropped.

2. If $\operatorname{dim} M \geqq 4, \mathscr{F}=\varnothing$, then $\mathscr{M}_{0}^{3}$ is still the union $\mathscr{M}_{0}^{3}=\left(\mathscr{M}_{0}^{s}-\mathscr{E}_{0}\right) \mathrm{U}$ $\mathscr{E}_{0}^{s}$, with $\mathscr{H}_{0}^{s}-\mathscr{E}_{0}^{s}$ a submanifold (I.2.2). But we do not know if $\mathscr{E}_{0}^{s}$, if nonempty, is a submanifold.

3. If $g(\lambda), \lambda \in(-\delta, \delta), \delta>0$, is a continuous curve in $\mathscr{H}_{0, s}^{s} g(0) \in \mathscr{F}^{s}$, then $g(\lambda)$ $\in F$, an interesting consequence of I.5.3.

If $g(\lambda)$ were a $C^{k}$ function of $\lambda, 1 \leqq k \leqq \infty$, then we could conclude from the method of 1.4.2 that all $k$ derivatives of $g(\lambda)$ at $\lambda=0$ are parallel, $\nabla\left(g^{(k)}(0)\right)=0$. Thus if $g(\lambda)$ is analytic, $g(\lambda)=\sum_{n=0}^{\infty}\left(\lambda^{0} / n !\right) g^{(n)}(0)$, and $\nabla\left(g^{(n)}(0)\right)=0$ for all $n$, so that $g(\lambda) \in \mathscr{F}^{3}$, a conclusion from 1.4 .2 alone.

4. Finally we remark that although $\mathscr{M}_{0}^{s}$ is a manifold (under the hypothesis $\mathscr{F}^{3}$ $\neq \varnothing$ ), the equation $R(g)=0$ is not linearization stable at a flat solution, as we have seen from the example in I.4. Here the dificulty can be traced to the fact that $\mathscr{M}_{0}^{z}$ is a union of closed manifolds of different "dimensionalities", $\mathscr{F}$ ' being essentially finite-dimensional modulo the orbit directions; cf. Example 1 in $\$ 0$. 


\section{Appljcation to General Relattvity}

I.1. The general set-up and main idea. We now turn our attention to the Einstein empty-space field equations of general relativity. We apply the techniques used in the previous sections to prove that solutions to these equations are linearization stable if certain conditions are met; in certain exceptional cases, however, the solutions are not linearization stable.

Let (1) $g$ be a smooth Lorentz metric (signature -+++ ) on a 4-manifold $V$. The Einstein empty-space field equations are that the Ricci tensor of ${ }^{(4)} g$ vanish:

$$
\operatorname{Ric}\left(\omega^{\prime}\right)=0 .
$$

An infinitesimal deformation about a solution ${ }^{(4)} g$ is then a solution (4) $h \in S_{2}$ of the linearized equations

$$
D \operatorname{Rig}(1) g) \cdot(1) h=0 \text {, }
$$

where $D$ Ric $\left({ }^{(4)} g\right)$ is the derivative of the map $\operatorname{Ric}(\cdot)$ at ${ }^{(1)} \mathrm{g}$.

Assume that $\left(V,{ }^{(4)} g\right)$ has a compact connected orientable spacelike hypersurface $M$ so that $\operatorname{dim} \boldsymbol{M}=3$. Let $g$ denote the induced riemannian metric on $M$ and $k$ the second fundamental form of $M$. Our conditions for linearization stability are as follows (see Note on p. 263):

$C_{x}:$ if $k=0, g$ is not flat;

$C_{d}:$ if $L_{X g}=0$ and $L_{X} k=0$, then $X=0$;

$C_{t}:$ tr $k=$ constant on $M$.

(The meaning of the subscripts $\mathscr{H}$ and $\delta$ will become clear.)

Our main result (II.S.1) is that if a solution $\left(V, \phi^{\prime}\right) g$ ) of (1) has a compact spacelike hypersurface $M$ whose induced metric $g$ and second fundamental form $k$ satisfy conditions $C_{x}, C_{\delta}$, and $C_{\mathrm{tr}}$, then every solution ${ }^{(4)} h$ of the linearized equations is tangent at ${ }^{(4)} g$ to a curve ${ }^{(4)} g(\lambda)$ of exact solutions of (1); i.e., there exist a tubular neighborhood $V^{\prime}$ of $M$ and a curve ${ }^{(4)} g(\lambda)$ of exact solutions of (1) on $V^{\prime}$ such that $\left.{ }^{\left({ }^{(4)}\right.} g(0),{ }^{(4)} g^{\prime}(0)\right)=\left(^{(4)} g,{ }^{(4)} h\right)$ on $V^{\prime}$; see also [16], [17], [39] and [40].

This conclusion asserts the linearization stability on a small piece of spacetime $V^{\prime}$ surrounding the Cauchy surface $M$. By standard arguments [12], $V^{\prime}$ can be extended to a maximal common development of the spacetimes ${ }^{(4)} g(\lambda), \lambda$ small, which approximates the maximal development of (4) $g(0)$.

The case where $V$ admits a noncompact spacelike hypersurface $M$ is rather different. Here asymptotic conditions are necessary. For example, $k=0$ and $g$ the usual flat metric on $\boldsymbol{R}^{3}$ is not excluded. Thus the usual Minkowski metric on $\boldsymbol{R}^{4}$ is linearization stable in a tubular neighborhood of the hypersurface $M=R^{3}$. This result was obtained independently using other methods by Choquet-Bruhat and Deser [10], [11]. The treatment of the general noncompact case is in spirit similar, although there are certain technical difficulties associated with elliptic operators on noncompact manifolds which enter the problem in the nonfiat case. We will present the noncompact case elsewhere. For the remainder of Part II, $M$ will be compact. 
It is convenient to introduce the supplementary variables $(g, \pi)$ instead of $(g, k)$, where $\pi=\pi^{\prime} \otimes \mu_{g} \in S^{2} \otimes \mu_{g}$ is a 2 -covariant symmetric tensor density, $\pi^{\prime}=$ ((tr $k) g-k)^{2} \in S^{2}$ is the tensor part of $\pi$ ( $k^{*}$ means the contravariant form of the tensor $k \in S_{2}$ ) and we write $\mu_{g}$ interchangeably with $d \mu_{g}$. In local coordinates

$$
\pi=\pi^{i j}=\left((\operatorname{tr} k) g^{i j}-k^{i j}\right)\left(\operatorname{det} g_{i j}\right)^{1 / 2} \text {. }
$$

As is easy to see, $k=0 \Leftrightarrow \pi=0$, tr $k=$ constant $\Leftrightarrow \operatorname{tr} \pi^{\prime}=$ constant, and $L_{x} g=0, L_{X} k=0 \Leftrightarrow L_{X} g=0, L_{X} \pi=0$. Thus, in the conditions $C_{x}, C_{\delta}$, and $C_{t r}, k$ can be replaced by $\pi$. Note that the divergence of $X$ enters in the Lie derivative of a teusor density:

$$
L_{X} \pi=\left(L_{X} \pi^{\prime}\right) \otimes \mu_{g}+\pi^{\prime} \otimes L_{X} \mu_{g}=\left(L_{X} \pi^{\prime}\right) \otimes \mu_{g}+\pi^{\prime} \otimes(\operatorname{div} X) \mu_{g}
$$

where div $X=-\delta X$. In local coordinates,

$$
L_{X} \pi=X^{k} \pi_{1 k}^{i j_{1 k}}-\pi^{i k} X^{j}{ }_{1 k}-\pi^{j k} X_{1 k}+X^{k}{ }_{1 k} \pi^{i j}
$$

As is well known every spacelike hypersurface in a Ricci-fiat Lorentz manifold ([1], [14]) satisfies the constraint equations

$$
\begin{aligned}
& \mathscr{H}(g, \pi)=\left\{\frac{1}{2}\left(\operatorname{tr} \pi^{\prime}\right)^{2}-\pi^{\prime} \cdot \pi^{\prime}+R(g)\right\} \mu_{g}=0, \\
& \delta(g, \pi)=\delta_{g} \pi=0 .
\end{aligned}
$$

In local coordinates,

$$
\mathscr{H}(g, \pi)=\left(\operatorname{det} g_{i j}\right)^{-1 / 2}\left\{\frac{1}{2}\left(g_{a b} \pi^{a b}\right)^{2}-\pi^{a b} \pi_{a b}\right\}+\left(\operatorname{det} g_{i j}\right)^{1 / 2} R(g)
$$

and

$$
\delta(g, \pi)=\delta_{g} \pi=-\pi^{i_{1}}{ }_{j}=0
$$

(so that $\delta_{g}$ now maps $S^{2} \rightarrow \mathscr{X} \otimes \mu_{g}$ ).

We shall refer to $\mathscr{H}(g, \pi)=0$ as the Hamiltonian constraint and $\delta_{g} \pi=0$ as the divergence constraint.

Conversely, by means of existence of solutions to the evolution equations (see, e.g., [15]), every solution $(g, \pi)$ to the constraint equations (C) generates a Riccifiat spacetime in a tubular neighborhood of $M$. This spacetime is unique up to diffeomorphism of the neighborhood.

For the compact hypersurface $M$, we let $\mathscr{M}^{s}$ denote the $H^{s}$ riemannian metrics on $M$; throughout, $s>n / 2+1$. For $g \in \mathscr{H}^{s}$, we have, as in Part $I, T_{g} \mathscr{A}^{s}=S_{2}^{s}$, and we let

$T_{8}^{*} \mathscr{H}^{s}=S_{2}^{s} \otimes \mu_{8}, \quad$ the space of $H^{s} 2$-covariant symmetric tensor densities, and

$$
T^{*} \cdot \mathscr{H}^{s}=\bigcup_{g \in s .} T_{g^{*}}^{*} \mathscr{U}^{s} \text {, the "cotangent" bundle of } \mathscr{H}^{s} \text {. }
$$

Note that here we take the dual in the $L_{2}$ inner product but use only the closed subspace of such elements continuous in the $H^{s}$ topology, so the dual of $S_{2}^{s}$ is $S_{3}^{2} \otimes$ $\mu_{g} ;$ see also [14, p. 552]. 
The solutions $(g, \pi)$ of the constraint equations may then be regarded as a certain subset $\mathscr{C}$ of $T^{*} \mathscr{A}^{3}$. We will, according to our general method, show that in a neighborhood of points $(g, \pi)$ that satisfy conditions $C_{x}, C_{z}$ and $C_{t r}, \mathscr{C}$ is a smooth submanifold of $T^{*} \mathscr{A l}^{\prime}$ and that if $(h, \omega) \in T_{(8, \pi)}\left(T^{*} \mathscr{H}^{\prime}\right) \approx S_{2}^{s} \times\left(S_{s}^{2} \otimes \mu_{8}\right)$ is tangent to $\mathscr{C}$, i.e., if $(h, \omega)$ is a solution to the linearized constraint equations, then there exist a $\delta>0$ and a smooth curve $(g(\lambda), \pi(\lambda)) \in \mathscr{C},-\delta<\lambda<\delta$, which is tangent to $(h, \omega)$ at $(g, \pi)$.

Now suppose ${ }^{(4)} h$ is a solution to the linearized equations, $D \operatorname{Ric}\left({ }^{(4)} g\right) \cdot\left({ }^{(4)} h=\right.$ 0. Then ${ }^{(4)} h$ induces a solution $(h, \omega)$ to the linearized constraint equations about a solution $(g, \pi)$ to the constraint equations. If $(g, \pi)$ satisfies conditions $C_{x}$, $C_{\delta}$, and $C_{t r}$, then there exists a curve $(g(\lambda), \pi(\lambda))$ of solutions to the full constraint equations. By using the existence theory for the Einstein equations this curve of solutions to the constraint equations generates a curve ${ }^{(4)} g(\lambda)$ of Ricci-flat Lorentz metrics (on a tubular neighborhood $V^{\prime}$ of $M$ ). After possible adjustment by a curve $\psi(\lambda): V^{\prime} \rightarrow V^{\prime}$ of diffeomorphisms of $V^{\prime},{ }^{(4)} g(\lambda)$ will be tangent to ${ }^{(4)} h\left({ }^{(4)} g(\lambda)\right.$ will be an $H^{s}$ spacetime and will be $\lambda$ differentiable in $H^{s-1}$ ); see also the end of $\$ 0$.

II.2. Solutions to the Hamiltonian constraint equation. For $s>n / 2+1$, we consider $\mathscr{H}: T^{*} \mathscr{M}^{s} \rightarrow A^{s-2},(g, \pi) \mapsto \mathscr{H}(g, \pi)$ as a map from $T^{*} \mathscr{H}^{s}$ to $A^{s-2}$, the $H^{s-2}$ 3-forms on $M$. For $g \in \mathscr{H}^{s}, A^{s-2}$ is isomorphic to $H^{s-2}(M ; R) \otimes \mu_{g}$ (by identifying $\lambda \in \Lambda^{s-2}$ with $\left.f \mu_{g}, f \in H^{s-2}(M ; R)\right)$.

Let $\mathscr{C}_{x}^{s}=\mathscr{H}^{-1}(0)=\left\{(g, \pi) \in T^{*} \mathscr{A}^{s}: \mathscr{H}(g, \dot{\pi})=0\right\}$, the solution set to the Hamiltonian constraint. Note that the set $\mathscr{F}^{s} \times\{0\}$ is a subset of $\mathscr{C}_{x}^{*}$, and that sina $\operatorname{dim} M=3, \mathscr{E}_{0}^{s}=\mathscr{F}^{s}$ (without the assumption that $\mathscr{F}^{\prime} \neq \varnothing$ ). Thus, from 1.3.3, $F^{*} \times\{0\}$ is a smooth submanifold of $T^{\star} \mathscr{A}$ (but is possibly empty). We will show that $\mathscr{F}^{s} \times\{0\}$ is the singular set on which $D \mathscr{H}(g, \pi)$ fails to be surjective. Thus

$$
\mathscr{C}_{x}^{s}=\left(\mathscr{C}_{x}^{s}-\mathscr{F}^{s} \times\{0\}\right) \cup\left(\mathscr{F}^{s} \times\{0\}\right)
$$

is the disjoint union of submanifolds. Thus $\mathscr{C}_{x}^{3}$ is somewhat similar to the structure of $\mathscr{A}_{0}^{3}$ (when $\mathscr{F}^{s} \neq \varnothing$ ); however, because of the kinetic terms involving the variable $\pi, \mathscr{F}^{3} \times\{0\}$ is not an isolated set of solutions of $\mathscr{P}(\mathrm{g}, \pi)=0$ (II.2.5); consequently, $\mathscr{C}_{x}^{2}-\mathscr{F}^{s} \times\{0\}$ need not be closed, and $\mathscr{S}_{\mathbb{R}}^{3}$ itself need not be a manifold.

To prove our result, the basic argument of $\mathbf{1 . 2 . 1}$ only has to be modified to take into account the kinetic terms:

II.2.1. TheOREM. Let $(g, \pi) \in \mathscr{C}_{\not R}^{s},(g, \pi) \notin \mathscr{F}^{s} \times\{0\}$. Then in a neighborhood of $(g, \pi), \mathscr{C}_{x}^{*}$ is a smooth submanifold of $T^{*} \cdot \mathbb{H}^{3}$, and $\mathscr{H}(g, \pi)=0$ is linearization stable at $(g, \pi)$.

Proof. We show that

$$
D \mathscr{H}(g, \pi): T_{(8, x)}^{*}\left(T^{*} \mathscr{H}^{s}\right) \approx S_{2}^{s} \times\left(S_{2}^{s} \otimes \mu_{g}\right) \rightarrow T_{0} A^{s-2} \approx A^{s-2} \approx H^{s-2} \otimes \mu_{g}
$$

is surjective for $(g, \pi) \in \mathscr{C}_{x}^{s}-\mathscr{F}^{s} \times\{0\}$, i.e., that $\mathscr{K}$ is a submersion at those $(g, \pi)$ that satisfy condition $C_{x}$.

By a straightforward computation, for $(h, \omega) \in S_{2}^{3} \times\left(S_{s}^{2} \otimes \mu_{8}\right)$, 


$$
\begin{aligned}
\gamma(g, \pi)(h, \omega)= & D \mathscr{H}(g, \pi) \cdot(h, \omega) \\
= & \left\{\Delta \operatorname{tr} h+\delta \delta h-h \cdot \operatorname{Ric}(g)+\frac{1}{2} \operatorname{tr} h R(g)\right. \\
& \left.+2\left(\frac{1}{2}\left(\operatorname{tr} \pi^{\prime}\right) \pi^{\prime}-\pi^{\prime} \times \pi^{\prime}\right) \cdot h-1\left(\frac{1}{2}\left(\operatorname{tr} \pi^{\prime}\right)^{2}-\pi^{\prime} \cdot \pi^{\prime}\right) \operatorname{tr} h\right\} \mu_{s} \\
& +2\left(\frac{1}{2}\left(\operatorname{tr} \pi^{\prime}\right) \operatorname{tr} \omega-\pi^{\prime} \cdot \omega\right),
\end{aligned}
$$

where $\pi^{\prime} \times \pi^{\prime}=\pi^{\prime i \pi} \pi^{\prime} d$ is the "product" of symmetric tensors.

Cantion. $D \mathscr{F}(g, \pi) \cdot(h, \omega)=D_{g} \mathscr{P}(g, \pi) \cdot h+D_{x} \mathscr{H}(g, \pi) \cdot \omega$, so that one must take the derivative of $\mathscr{H}$ with respect to $\pi$ (which is best dcre by using the coordinate expression involving (det $\left.g_{\theta}\right)^{-1 / 2}$ and not $\pi^{\prime}$, although t' $2 e$ final expression is in terms of $\pi$ ).

The $L_{z}$ adjoint, $\tau_{(s, x)}^{*}: H^{s} \otimes \mu_{z} \rightarrow S_{2}^{s-2} \times\left(S_{s}^{2} \otimes \mu_{s}\right)$ is then given by

$$
\begin{aligned}
\tau_{(g, x)}^{*}\left(N \mu_{g}\right)= & \left(g \Delta N+\operatorname{Hess} N-N \operatorname{Ric}(g)+\frac{1}{2} N R(g) g\right. \\
& +\left\{2\left(\frac{1}{2}\left(\operatorname{tr} \pi^{\prime}\right) \pi^{\prime}-\pi^{\prime} \times \pi^{\prime}\right)^{b}-\frac{1}{2}\left(\frac{1}{2}\left(\operatorname{tr} \pi^{\prime}\right)^{\prime}-\left(\pi^{\prime} \cdot \pi^{\prime}\right)\right) g\right\} N, \\
& \left.2\left(\frac{1}{2}(\operatorname{tr} \pi) g^{2}-\pi\right) N\right) .
\end{aligned}
$$

Since the symbol $\sigma_{\epsilon_{0}}\left(\gamma_{(x-x)}^{*}\right): R \rightarrow\left(T_{x}^{*} M \otimes_{2 y \mathrm{~m}} T_{x}^{*} M\right) \times\left(T_{x} M \otimes_{s y \mathrm{~m}} T_{x} M\right)$ is given

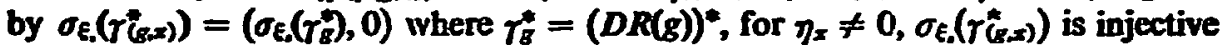
by the first factor alone. Thus we must show that $T_{(x, x)}^{*}$ is injective. Thus, let $T\left(\sigma_{x x}\right)\left(N_{f_{g}}\right)=0$, so that

$$
\begin{gathered}
g \Delta N+\text { Hess } N-N^{b} \operatorname{Riq}(g)+\frac{1}{2} N R(g) g \\
\left.+\left\{2\left(\frac{1}{2(t r} \pi^{\prime}\right) \pi^{\prime}-\pi^{\prime} \times \pi^{\prime}\right)^{b}-\frac{1}{2}\left(\frac{1}{2}\left(\operatorname{tr} \pi^{\prime}\right)^{2}-\pi^{\prime} \cdot \pi^{\prime}\right) g\right\} N=0 ; \\
2\left(\frac{1}{2}(\operatorname{tr} \pi) g^{\prime}-\pi\right) N=0 .
\end{gathered}
$$

Taking the trace of (b) gives (tr $\pi) N=0$, and so again from (b), $N \pi=0$. Since $\mathscr{H}(g, \pi)=0$ and $N \pi=0, N R(g)=0$. Thus (a) simplifies to

(c)

$$
g \Delta N+\operatorname{Hess} N-N \operatorname{Ric}(g)=0 \text {, }
$$

which; as in the proof of 1.2 .1 , implies that $N=0$ unless $R$ ic $(g)=0 \Leftrightarrow g$ is fiat for $\operatorname{dim} M=3$. If $g$ is fiat, taking the trace of (c) gives $\Delta N=0$ so that $N$ is a constant. Thus from $N \pi=0, N=0$ unless $\pi=0$. Thus, if $g$ is fiat and $\pi \neq 0, N=0$, so that $\gamma^{*}(g, x)$ is injective and $\gamma(r, x)$ is surjective.

Thus $\mathscr{H}$ is singular (in the sense that $D \mathscr{H}(g, \pi)$ is not surjective) on the set $\mathscr{F} \times$ $\{0\}$, and on this set ker $T_{(g . x)}^{*}=$ \{constant functions on $A: ?$. Thus we expect the equation $\mathscr{H}(g, \pi)=0$ to be linearization unstable in a neighborhood of a solution $(g F, 0) \in F \times\{0\}$. To find the extra condition implied ty this instability, we introduce the integrated Hamiltonian density (= the Hamiltonian)

$$
H: T^{*} \mathscr{H}^{3} \rightarrow R, \quad(g, \pi) \mapsto \int \mathscr{H}(g, \pi)=\int\left\{\frac{1}{2}\left(\mathrm{tr} \pi^{2}\right)^{2}-\pi^{\prime} \cdot \pi^{\prime}+R(g)\right\} d \mu_{s}
$$

the total kinetic energy,

$$
K: T^{*} \mathscr{A}^{3} \rightarrow R, \quad(g, \pi) \mapsto \int\left(1\left(\operatorname{tr} \pi^{\prime}\right)^{2}-\pi^{\prime} \cdot \pi^{\prime}\right) d \mu_{B}
$$

and the integrated scalar curvature

$$
\Phi: \mathscr{A}^{s} \rightarrow R, \quad g \mapsto \int R(g) d \mu_{5}
$$


252

ARTHUR E. FISCHER AND JERROLD E. MARSDEN

Thus $H(g, \pi)=K(g, \pi)+\Phi(g)$, and $\Phi$ serves as a potential for the Hamiltonian; see [14] for the geometrical consequences of this interpretation. First we consider the map $\Phi$ (see also [3]).

II.2.2. Theorem. Let $\operatorname{dim} M \geqq 3$. Then a metric $g \in \mathscr{M}^{*}$ is a critical point of $\Phi$ if and only if $\operatorname{Ric}(g)=0$. At a critical point $g \in \mathscr{M}^{s}$, the Hessian of $\Phi$ is

$$
\begin{aligned}
d^{2} \Phi(g) \cdot\left(h_{1}, h_{2}\right)= & -\frac{1}{2} \int h_{1} \cdot \Delta_{L} h_{2} d \mu_{g}+\int \delta h_{1} \cdot \delta h_{2} d \mu_{g}+\frac{1}{2} \int \delta h_{1} \cdot d \operatorname{tr} h_{2} d \mu_{8} \\
& +\frac{1}{2} \int d \operatorname{tr} h_{1} \cdot \delta h_{2} d \mu_{g}+\frac{1}{2} \int d \operatorname{tr} h_{1} \cdot d \operatorname{tr} h_{1} d \mu_{g} .
\end{aligned}
$$

If $g \in \mathscr{M}^{s+1}$ so that $h \in S_{2}^{s}$ can be decomposed as $h=h+L_{X g}$ (where $h \in S_{2}^{\xi}$ is the divergence free part of $h$ ), then

$$
d^{2} \Phi(g) \cdot\left(h_{1}, h_{2}\right)=-\frac{1}{2} \int h_{1} \cdot \Delta_{L} h_{2} d \mu_{g}+\frac{1}{2} \int d \operatorname{tr} h_{1} \cdot d \operatorname{tr} h_{2} d \mu_{g}
$$

If $g_{F} \in \mathcal{F}^{2}$ (so that $h \in S_{2}^{z}$ has a decomposition $h=h+L_{X g}$ ) then $\Delta h h=\Delta h$, and

$$
d^{2} \phi\left(g_{F}\right) \cdot(h, h)=-\frac{1}{2} \int(\nabla h)^{2} d \mu_{z}+\frac{1}{2} \int(d \operatorname{tr} h)^{2} d \mu_{B}
$$

for a flat riemannian metric $g_{F}$.

Proof. First we find the critical points of $\Phi$. We compute the derivative of $\Phi$ :

$$
\begin{aligned}
d \Phi(g) \cdot h & =D \Phi(g) \cdot h=\int D R(g) \cdot h d \mu_{z}+\int R(g) D\left(d \mu_{g}\right) \cdot h \\
& =\int(\Delta \operatorname{tr} h+\delta \delta h-h \cdot \operatorname{Ric}(g)) d \mu_{g}+\int R(g) \frac{1}{2} \operatorname{tr} h d \mu_{g} \\
& =-\int\left(\operatorname{Ric}(g)-\frac{1}{2} g R(g)\right) \cdot h d \mu_{g}
\end{aligned}
$$

since $\int(\Delta \operatorname{tr} h+\delta \delta h) d \mu_{g}=0$ for all $(g, h)$ by Stokes' theorem. Thus $d \Phi(g) \cdot h=0$ for all $h \in S_{2}^{s} \Leftrightarrow \operatorname{Ric}(g)-\frac{1}{2} g R(g)=0$. Since $\operatorname{dim} M \geqq 3$, by considering the trace of this we see that it is equivalent to $\operatorname{Ric}(g)=0$.

From (1), the second derivative of $\Phi$ is

$$
\begin{aligned}
d^{2} \Phi(g) \cdot(h, h)=\int & D^{2} R(g) \cdot(h, h) d \mu_{g} \\
& +2 \int(D R(g) \cdot h)\left(D\left(d \mu_{g}\right) \cdot h\right)+\int R(g) D^{2}\left(d \mu_{g}\right) \cdot(h, h) .
\end{aligned}
$$

At a critical point $g, \operatorname{Ric}(g)=0, R(g)=0$, and $D R(g) \cdot h=\Delta \operatorname{tr} h+\delta \delta h$, and so from I.4.1, (2) becomes

$$
\begin{aligned}
d^{2} \Phi(g) \cdot(h, h)=- & -\frac{1}{2} \int h \cdot \Delta_{L} h d \mu_{g}-\frac{1}{2} \int(d \operatorname{tr} h)^{2} d \mu_{g}+\int(\delta h)^{2} d \mu_{g} \\
& +2 \int(\Delta \operatorname{tr} h+\delta \delta h)\left(\frac{1}{2} \operatorname{tr} h\right) d \mu_{g} \\
=- & \frac{1}{2} \int h \cdot \Delta_{L} h d \mu_{g}+\frac{1}{2} \int(d \operatorname{tr} h)^{2} d \mu_{g}+\int(\delta h)^{2} d \mu_{g}+\int d \operatorname{tr} h \cdot \delta h d \mu_{g}
\end{aligned}
$$


If $g \in \mathscr{L}^{3+1}, \operatorname{Rid}(g)=0$, and $h=h+L_{x} g$, then $D R(g) \cdot h=D R(g) \cdot h=\Delta \operatorname{tr} h$, and so

$$
\begin{aligned}
d^{2} \Phi(g) \cdot(h, h)= & -\frac{1}{2} \int h \cdot \Delta_{L} h d \mu_{z}-\frac{1}{2} \int(d \operatorname{tr} h)^{2} d \mu_{g} \\
& +2 \int d \operatorname{tr} h \cdot d \delta X d \mu_{z}+\int(\Delta \operatorname{tr} h) \operatorname{tr} h d \mu_{g} \\
= & -\frac{1}{2} \int h \cdot \Delta_{L} h d \mu_{g}-\frac{1}{2} \int(d \operatorname{tr} h)^{2} d \mu_{z} \\
& +\int d \operatorname{tr} h \cdot(2 d \delta X+d \operatorname{tr} h) d \mu_{g} \\
= & -\frac{1}{2} \int h \cdot \Delta h d \mu_{z}+\frac{1}{2} \int(d \operatorname{tr} h)^{2} d \mu_{z}
\end{aligned}
$$

since tr $h=\operatorname{tr} h-2 \delta X$ so that $d \operatorname{tr} h=d \operatorname{tr} h+2 d \delta X$.

Rararks.1. The change to a positive sign of the $\frac{1}{2} \int(d \text { tr } h)^{2} d \mu_{g}$ term in $d^{2} \phi(g)$. $(h, h)=d^{2}\left(\int R(g) d \mu_{g}\right) \cdot(h, h)$ as compared with $\int D^{2} R(g) \cdot(h, h) d \mu_{g}$ comes about because of the term $2 \int(D R(g) \cdot h)\left(D\left(d \mu_{g}\right) \cdot h\right)$ involving the derivative of the volume element. Because of this sign change, a flat metric $g_{F}$ is a saddle point for $d^{2} \phi\left(g_{F}\right)$ (even within a slice), whereas $\int D^{2} R(g) \cdot(h, h) d \mu_{g} \leqq 0$ on a slice at $g_{F}$. Thus because of this sign change, the behavior of the integrated scalar curvature $\Phi(g)=\int R(g) d \mu_{z}$ is somewhat different from the pointwise scalar curvature $R(g)$ at $g_{F}$.

2. That $d^{2} \Phi(g) \cdot(h, h)=d^{2} \Phi(g) \cdot(h, h)$ depends only on the divergence free part of $h$ follows from the invariance of $\Phi$ by $\mathscr{D}$, so that $d^{2} \Phi(g) \cdot\left(L_{X g}, L_{X g}\right)=0$, and by orthogonality of $h$ and $L_{X g}$, so that $d^{2} \Phi(g) \cdot\left(h, L_{X g}\right)=0$.

We can now easily compute the critical points and the Hessian of the Hamiltonian $H: T^{*} \mathscr{M}^{s} \rightarrow R$. From now on, $\operatorname{dim} M=3$.

11.2.3. Throirem. A pair $(g, \pi) \in T^{*} \mathscr{H}^{3}$ is a critical point of $H: T^{*} \mathscr{H}^{3} \rightarrow R,(g, \pi)$ $\mapsto \int \mathscr{H P}(g, \pi)$ if and only if $(g, \pi) \in \mathscr{F}^{3} \times\{0\}$.

At a critical point $\left(g_{F}, 0\right)$, the Hessian of $H$ is

$$
\begin{aligned}
d^{2} H\left(g_{F}, 0\right) \cdot((h, \omega),(h, \omega))=- & \frac{1}{2} \int(\nabla h)^{2} d \mu_{g}+\frac{1}{2} \int(d \operatorname{tr} h)^{2} d \mu_{g} \\
& +2 \int\left(\frac{1}{2}\left(\operatorname{tr} \omega^{\prime}\right)^{2}-\omega^{\prime} \cdot \omega^{\prime}\right) d \mu_{g} .
\end{aligned}
$$

Proop. From the computation in the proof of II.2.1,

$$
\begin{aligned}
d H(g, \pi) \cdot(h, \omega)= & \int D \mathscr{H}(g, \pi) \cdot(h, \omega) \\
= & -\int\left(\operatorname{Rio}(g)-\frac{1}{2} g R(g)\right) \cdot h d \mu_{g}+2 \int\left(\frac{1}{2}\left(\operatorname{tr} \pi^{\prime}\right) \pi^{\prime}-\pi^{\prime} \times \pi^{\prime}\right) \cdot h d \mu_{g} \\
& -\frac{1}{2} \int\left(\frac{1}{2}\left(\operatorname{tr} \pi^{\prime}\right)^{2}-\pi^{\prime} \cdot \pi^{\prime}\right) \operatorname{tr} h d \mu_{g}+2 \int\left(\frac{1}{2}\left(\operatorname{tr} \pi^{\prime}\right) \operatorname{tr} \omega-\pi^{\prime} \cdot \omega\right)=0
\end{aligned}
$$

for all $(h, \omega) \in S_{s}^{2} \times\left(S_{s}^{2} \otimes \mu_{g}\right)$. Thus

(a) $-\left(\operatorname{Rid}(g)-\frac{1}{2} g R(g)\right)+2\left(\frac{1}{2}\left(\operatorname{tr} \pi^{\prime}\right) \pi^{\prime}-\pi^{\prime} \times \pi^{\prime}\right)-\frac{1}{2}\left(\frac{1}{2}\left(\operatorname{tr} \pi^{\prime}\right)^{2}-\pi^{\prime} \cdot \pi^{\prime}\right) g=0$,

$$
\frac{1}{2}\left(\operatorname{tr} \pi^{\prime}\right) g-\pi^{\prime}=0 \text {. }
$$


Contracting (b) gives tr $\pi^{\prime}=0$, and thus from (b) again, $\pi^{\prime}=0$ (and so $\pi=0$ ). Thus, from (a), $\operatorname{Ric}(g)-\frac{1}{2} g R(g)=0 \Rightarrow \operatorname{Ric}(g)=0 \Rightarrow g$ is fiat.

In the computation for

$$
\begin{aligned}
d^{2} H(g, \pi) \cdot(h, h)= & d^{2}\left(\int R(g) d \mu_{z}\right) \cdot(h, h) \\
& +d^{2}\left(\int\left(\frac{1}{2}\left(\operatorname{tr} \pi^{\prime}\right)^{2}-\pi^{\prime} \cdot \pi^{\prime}\right) d \mu_{z}\right) \cdot((h, \omega),(h, \omega)),
\end{aligned}
$$

the terms due to the kinetic part of $H$ are straightforward to compute; since $\pi=0$ and the kinetic part is quadratic in $\pi$,

$$
\begin{aligned}
d^{2} K(g, 0) \cdot((h, \omega)(h, \omega)) & =d^{2}\left(\int\left(\frac{1}{2}\left(\operatorname{tr} \pi^{\prime}\right)^{2}-\pi^{\prime} \cdot \pi^{\prime}\right) d \mu_{g}\right) \cdot((h, \omega),(h, \omega)) \\
& =2 \int\left(\frac{1}{2}\left(\operatorname{tr} \omega^{\prime}\right)^{2}-\omega^{\prime} \cdot \omega^{\prime}\right) d \mu_{g} .
\end{aligned}
$$

The expression for $d^{2}\left(\int R(g) d \mu_{g}\right) \cdot(h, h)$ is given by II.2.2.

Note that the critical points of $H$ are exactly the set where $\mathscr{P}$ is singular; i.e. where $D \mathscr{P}(g, \pi)$ is not surjective. This "coincidence" follows from the fact that

$$
d H(g, \pi) \cdot(h, \omega)=\int D \mathscr{H}(g, \pi) \cdot(h, \omega)=\int\left((D \mathscr{H}(g, \pi))^{*}\right) \cdot(h, \omega)=0
$$

for all $(h, \omega) \in T_{(g x)}\left(T^{*} \mathscr{A}^{3}\right)$ iff $1 \in \operatorname{ker}(D \mathscr{P}(g, \pi))^{*}$ iff $D \mathscr{P}(g, \pi)$ is not surjective.

Around $(g, 0) \in \mathscr{J} \times\{0\}$, the equation $\not{H}(g, \pi)=0$ is linearization unstableThe extra condition that a first order deformation $(h, \omega)$ must satisfy for it to be tangent to a curve $(g(\lambda), \pi(\lambda))$ of exact solutions of $\mathscr{H}(g, \pi)=0$ is given by the following:

I1.2.4. Theorem. Let $(g(\lambda), \pi(\lambda)) \in \mathscr{C}_{\Sigma}^{s} \subset T^{*} A^{s}, \lambda \in(-\delta, \delta), \delta>0$, be a $C^{2}$ curve with $(g(0), \pi(0))=\left(g_{F}, 0\right) \in F^{3} \times\{0\},\left(g^{\prime}(0), \pi^{\prime}(0)\right)=(h, \omega)$. Then $(h, \omega)$ must satisfy $\operatorname{tr} h=$ constant and

$$
-\frac{1}{2} \int(\nabla h)^{2} d \mu_{g,}+2 \int\left(\frac{1}{2}\left(t+\omega^{\prime}\right)^{2}-\omega^{\prime}-\omega^{\prime}\right) d \mu_{z_{r}}=0
$$

where $h$ is the divergence free part of $h$.

Proof. Differentiating $\mathscr{H}(g(\lambda), \pi(\lambda))=0$ twice and evaluating at $\lambda=0$, we have

$$
\begin{aligned}
\left.\frac{d \mathscr{H}}{d \lambda}(g(\lambda), \dot{\pi}(\lambda))\right|_{\lambda=0} & =\left.D \mathscr{H}(g(\lambda), \pi(\lambda)) \cdot\left(g^{\prime}(\lambda), \pi^{\prime}(\lambda)\right)\right|_{\lambda=0} \\
& =D \mathscr{H}(g F, 0) \cdot(h, \omega)=(\Delta \operatorname{tr} h+\delta \delta h) d \mu_{g} \\
& =(\Delta \operatorname{tr} h) d \mu_{g}=0
\end{aligned}
$$

and

$$
\begin{aligned}
\left.\frac{d^{2} \mathscr{H}}{d \lambda^{2}}(g(\lambda), \pi(\lambda))\right|_{\lambda=0}= & \left.D^{2} \mathscr{H}(g(\lambda), \pi(\lambda)) \cdot\left(\left(g^{\prime}(\lambda), \pi^{\prime}(\lambda)\right),\left(g^{\prime}(\lambda), \pi^{\prime}(\lambda)\right)\right)\right|_{2=0} \\
& +\left.D \mathscr{H}(g(\lambda), \pi(\lambda)) \cdot\left(g^{\prime \prime}(\lambda), \pi^{\prime \prime}(\lambda)\right)\right|_{\lambda=0} \\
= & D^{2} \mathscr{H}\left(g_{F}, 0\right) \cdot((h, \omega),(h, \omega)) \\
& +D_{H}\left(g_{F}, 0\right) \cdot\left(g^{\prime \prime}(0), \pi^{\prime \prime}(0)\right)=0 .
\end{aligned}
$$


Integrating (2) over $M$ gives

$$
d^{2} H\left(g_{F}, 0\right) \cdot((h, \omega),(h, \omega))=\int D^{2} \mathscr{H}\left(g_{F}, 0\right) \cdot((h, \omega),(h, \omega))=0
$$

since $\left(g_{F}, 0\right)$ is a critical point of $H$, so that

$$
d H\left(g_{F}, 0\right) \cdot\left(g^{\prime \prime}(0), \pi^{\prime \prime}(0)\right)=\int D \mathscr{H}\left(g_{F}, 0\right) \cdot\left(g^{\prime \prime}(0), \pi^{\prime \prime}(0)\right)=0 .
$$

From the first order condition (1), tr $h=$ constant, so that from 11.2 .3 , the second order condition (3) becomes

$$
-\frac{1}{2} \int(\nabla h)^{2} d \mu_{g}+2 \int\left(\frac{1}{2}\left(\operatorname{tr} \omega^{\prime}\right)^{2}-\omega^{\prime} \cdot \omega^{\prime}\right) d \mu_{g}=0
$$

Remarks. 1. The first order condition (1) does not give any restrictions on $\omega$. However in the next section we shall see that the first order deformation $(h, \omega)$ of the $\delta_{g} \pi=0$ constraint around $\pi=0$ implies that $\delta \omega=0$.

2. As an example of a nonintegrable deformation, let $\left(T^{3}, g_{F}\right)$ and $h$ be as in the example of I.4. Then if $\omega=0,(h, 0)$ satisfies the linearized Hamiltonian constraint but not the second order condition, which for $\omega=0$ reduces to $\nabla h=0$. Thus $(h, 0)$ cannot be tangent to any curve $(g(\lambda), \pi(\lambda)) \in \mathscr{C}_{x}$.

Even though $g_{F}$ is fiat, the integrated extra condition on a first order deformation $(h, \omega)$ cannot be converted to a pointwise condition as in 1.4 .2 or the above remark since the kinetic term $\int\left(\frac{1}{2}\left(\operatorname{tr} \omega^{\prime}\right)^{2}-\omega^{\prime} \cdot \omega^{\prime}\right) d \mu_{g}$ is not negative-definite, even if the condition $\delta \omega=0$ is imposed. Not being able to convert to a pointwise extra condition signals that although there is linearization instability of $\mathscr{H}(g, \pi)=$ 0 at $\left(g_{F}, 0\right) \in \mathscr{F}^{-} \times\{0\}$, these solutions are not isolated solutions. In fact, if we ignore the $\delta \pi=0$ constraint, we can construct solutions algebraically to $\mathscr{K}(g, \pi)=$ $0,(g, \pi) \notin F^{s} \times\{0\}$, which are arbitrarily close to a solution $(g F, 0) \in \mathcal{F}^{s} \times\{0\}$.

This construction proceeds as follows: let $A^{T} \in S^{2}$ be any traceless tensor, $\operatorname{tr} A^{T}=0$, and let $\pi_{\varepsilon}=E\left(A^{T}+\left((2 / 3) A^{T} \cdot A^{T}\right)^{1 / 2} g_{F}\right) \mu_{g} ;$ here the trace and pointwise contraction "." are with respect to $g F$. Then

$$
\mathscr{H}\left(g_{F}, \pi_{\varepsilon}\right)=\left(R\left(g_{F}\right)+\frac{1}{2}\left(\operatorname{tr} \pi_{\varepsilon}^{\prime}\right)^{2}-\pi_{\varepsilon}^{\prime} \cdot \pi_{\varepsilon}^{\prime}\right) \mu_{g,}=0 .
$$

Thus for $\varepsilon$ small, $\pi_{\varepsilon}$ can be made arbitrarily close to 0 .

In this construction, $\delta \pi_{\varepsilon} \neq 0$, and this situation cannot be remedied by choosing $A^{T}$ to be transverse (i.e. $\left.\delta A^{T}=0\right)$ as well as traceless, since $\phi=\left((2 / 3) A^{T} \cdot A^{T}\right)^{1 / 2}$ need not be constant, so that $\pi=\left(A^{T}+\phi g_{F}\right) \mu_{g}$, need not be divergence free.

However, by being more subtle, we can still construct solutions to $\mathscr{H}(g, \pi)=0$, $\delta_{g} \pi=0,(g, \pi) \notin \mathscr{F}^{s} \times\{0\}$, which are arbitrarily close to the manifold of solutions Fs: $\times\{0\}$.

II.2.5. TheoreM. Let $\left(g_{F}, 0\right) \in \mathscr{F}^{s} \times\{0\}$. Then in every neighborhood $U_{(g, 0)}^{s} \subset$ $T^{*} \mathscr{A}^{s}$ of $(g F, 0)$, there exists $a(g, \pi) \in U_{(g, n)}^{s}$, such that $(g, \pi) \notin \mathscr{F}^{s} \times\{0\}, \mathscr{H}(g, \pi)$ $=0, \delta_{g} \pi=0$, and $\operatorname{tr} \pi=c \mu_{g}, c=$ constant $\neq 0$. 
Proor. We use a stability argument based on the Lichnerowicz [24] and ChoquetBruhat [7], [8], [9] conformal method of constructing solutions to the constraint equations.

For $g_{0} \in \mathscr{A}^{2}$, let $A^{T T} \in S_{s}^{2}$ be such that $\delta_{g_{0}} A^{T T}=0$ and tr $A^{T T}=0$. Let $M=$ $A^{T T} \cdot A^{T T}$ ("." is with respect to $g_{0}$ ) and let $c=$ constant. Then from [8], [9] (see also [33] for the case $c=$ constant $\neq 0)$, if $M \neq 0\left(\Leftrightarrow A^{T T} \neq 0\right), c \neq 0$, there exists a unique $\phi \in H^{\prime}(M ; R), \phi>0$, that satisfies the Lichnerowicz equation

$$
8 \Delta \phi=-R(g) \phi+M \phi^{-7}-\frac{1}{6} c^{2} \phi^{5} \text {. }
$$

Moreover, if $g=\phi^{4} g_{0}$

$$
\pi=\left(\phi^{-4} A^{T T}+(c / 3) \phi^{2} g_{0}^{2}\right) \mu_{g_{0}}=\left(\phi^{-10} A^{T T}+(c / 3) g^{2}\right) \mu_{z}
$$

(where the last equality follows from $g^{s}=\phi^{-4} g_{0}^{5}$ and $\left.\mu_{g}=\phi^{6} \mu_{z^{3}}\right)$, then $\mathscr{H}(g, \pi)=$ $0, \delta_{g} \pi=0$, and $t r \pi=c \mu_{g}$

The stability theorem of [9], adapted to the case that $c \neq 0$, proves that solutions to $(\mathrm{L})$ are stable with respect to $g, M$, and $c^{2}$, if $M \neq 0$, and $c \neq 0$; i.e. if we let

$$
Y: T^{*} \mathscr{M}^{s} \rightarrow H^{s}(M ; R), \quad(\mathrm{g}, \pi) \mapsto \phi
$$

be defined for those $(g, \pi) \in T^{*} \mathscr{A}^{s}$ such that $\pi$ is of the form $\pi=\left(A^{T T}+(c / 3) g\right) \mu_{g}$, $A^{T T} \neq 0, c \neq 0$, and let $\phi=Y(g, \pi) \in H^{s}(M ; R), \phi>0$, be defined as the unique solution of $(L)$, then $Y$ is a continuous map.

From the uniqueness theorem for $(L)$, if

$$
-R(g)+M-\frac{1}{6} c^{2}=0, \quad M \neq 0, c \neq 0,
$$

then $\phi \equiv 1$ is the unique solution of (L). But then from stability of solutions to (L), it follows that if $-R(g)+M-(1 / 6) c^{2}(M \neq 0, c \neq 0)$ is $H^{s-2}$ close to $0 \in H^{s-2}(M ; R)$, then the unique solution $\phi>0$ of $(\mathrm{L})$ is $H^{2}$ close to $1 \in H^{2}(M ; R)$; i.e. if $U_{1}^{z} \subset H^{2}(M ; R)$ is a neighborhood of 1 , there exists a neighborhood $U_{0}^{j-2} \subset H^{s-2}(M ; R)$ of 0 such that if $-R(g)+M-(1 / 6) c^{2} \in U_{0}^{s-2}, M \neq 0, c \neq 0$, then the unique solution $\phi>0$ of $(\mathrm{L})$ is in $U_{1}^{3}$.

Now let

$$
\begin{aligned}
& \nabla_{(g n 0)}^{g}=\left\{(g, \pi) \in U_{(g \cap 0)}^{3}:\right. \text { if } \pi=\left(A^{T T}+(c / 3) g\right) \mu_{g} \text { and } \phi \in U_{j}^{s}, \phi>0, \\
&\text { then } \left.\left(\phi^{4} g,\left(\phi^{-4} A^{T T}+(c / 3) \phi^{2} g^{*}\right) \mu_{g}\right) \in U_{(g m 0)}^{s}\right\},
\end{aligned}
$$

and

$$
\hat{U}_{(g, 0)}^{s}=\left\{(g, \pi) \in U_{(g, 0)}^{s}: \begin{array}{l}
\text { if } \pi=\left(A^{T T}+(c / 3) g\right) \mu_{g}, \\
\text { then } \left.-R(g)+M-f c^{2} \in U_{0}^{z-2}\right\},
\end{array}\right.
$$

so that $U_{(g, 0)}^{s} \subset U_{(g, 0)}^{s}$ and $\hat{U}_{(g, 0)}^{s} \subset U_{(g, 0)}^{s}$ are both neighborhoods of $\left(g_{F}, 0\right)$.

Now let $\left(g_{0}, \pi_{0}\right) \in U_{(g, 0)}^{3} \cap \hat{U}_{(g, 0)}^{(g, 0)}$ with $\pi_{0}$ of the form $\pi_{0}=\left(A^{T T}+(c / 3) g_{0}^{t}\right) \mu_{g_{0},}$ $A^{T r} \neq 0, c \neq 0$, and let $\phi>0$ be the unique solution of (L) with coefficients $\left(g_{0}, M, c\right)$. From the construction of $\hat{U}_{(g, 0)}^{s}$ and $U_{0}^{s-2}, \phi \in U_{1}^{s}$, so from the construction of $\bar{U}_{(g, 0)}^{s}$, if $g=\phi^{4} g_{0}, \pi=\left(\phi^{-4} A^{T T}+(c / 3) \phi^{2} g_{0}^{*}\right) \mu_{g 0},(g, \pi) \in U_{(g, 0)}^{s}$, and $\mathscr{H}(g, \pi)=0, \delta_{g} \pi=0$, tr $\pi=\phi^{4} g_{0} \cdot \pi=c \phi^{6} \mu_{g_{0}}=c \mu_{g}, c \neq 0$, and $(g, \pi) \notin \ldots$ $F^{*} \times\{0\}$ 
Thus the set $\mathscr{F} \times\{0\}$ is not isolated among the solutions of $\mathscr{H}(g, \pi)=0$ and $\delta_{g} \pi=0$. In fact, in [6], Brill-Deser show by example that a flat 3-torus and $\pi=$ $\mathbf{0}$ is not an isolated solution of the constraint equations.

As shall be apparent from II.3.2, the divergence constraint also does not have any isolated solutions. Thus because $\mathscr{H}(g, \pi)=0$ is linearization stable at all $(g, \pi) \notin \mathscr{F} \times\{0\}$, we can conclude:

There are no isolated solutions of the empty-space constraint equations of general relativity.

This result also holds for all physically reasonable stress-energy tensors.

Interestingly, if we look for solutions to the constraint equations that also satisfy the condition tr $\pi=0$, then $\mathscr{F}^{s} \times\{0\}$ is an isolated manifold of solutions to the Hamiltonian constraint (and hence to both constraint equations).

11.2.6. THEOREM. Let $\left(g_{F}, 0\right) \in F^{8} \times\{0\}$. Then there exists a neighborhood $U_{\left(g_{n} 0\right)}$ $\subset T^{*} \mathscr{A}^{s}$ of $\left(g_{F}, 0\right)$ such that if $(g, \pi) \in U_{(g, 0)}, \mathscr{H}(g, \pi)=0$, and $\operatorname{tr} \pi=0$, then $(g, \pi) \in g^{3} \times\{0\}$.

Proof. From I.5.2 there exists a neighborhood $U_{g r} \subset \mathscr{M}^{s}$ such that if $g \in U_{g n}$ $R(g) \geqq 0$, then $g \in F^{s}$. Let $U_{(g, 0)}=T^{*} U_{g,} \subset T^{*} M^{s}$. Then if $(g, \pi) \in U_{(g, 0)}$, $\mathscr{H}(g, \pi)=0$, tr $\pi=0$, then $R(g)=\pi^{\prime} \cdot \pi^{\prime} \geqq 0$, and since $g \in U_{g n} g \in \mathscr{F}^{s}$, so $R(g)$ $=0$, and hence $\pi=0$.

REMARKs. 1. In particular, the solutions $\mathscr{F}^{3} \times\{0\}$ are isolated among the timesymmetric $(\pi=0)$ solutions to the Hamiltonian constraint.

2. The variation of the $\operatorname{tr} \pi=0$ condition is

$$
D(\operatorname{tr} \pi) \cdot(h, \omega)=h \cdot \pi+\operatorname{tr} \omega=0 .
$$

Thus if $\pi=0$, a deformation of tr $\pi=0$ must satisfy tr $\omega=0$. Using this condition, the second order condition of II.2.4 reduces to the pointwise condition $\nabla \hat{h}$ $=0$ and $\omega=0$. This is the basis of the "second order" version of II.2.6, proven in [5].

Although II.2.6 proves isolation in a full neigi:borhood of $\mathscr{F}^{s} \times\{0\}$, in light of 11.2 .5 , the isolation of this set is more a consequence of the $\operatorname{tr} \pi=0$ condition than of the constraint equations.

II.3. The divergence constraint. Now let $\delta: T^{*} \mathscr{M}^{s} \rightarrow X^{s-1} \times V^{s},(g, \pi) \mapsto \delta_{R} \pi=$ $-\pi^{i j}{ }_{1 j}$, where $\boldsymbol{\gamma}^{s}$ is the set of $H^{s}$ volume elements on $M$. Let

$$
\mathscr{C}_{\delta}^{s}=\delta^{-1}(0)=\left\{(g, \pi) \in T^{*} \mathscr{H}^{3}: \delta(g, \pi)^{*}=0\right\}
$$

denote the set of solutions to the divergence constraint.

11.3.1. Theorem. $L_{e t}(g, \pi) \in \mathscr{C}_{\delta}^{\prime}$ satisfy condition $C_{\delta}:$ if $L_{X} g=0$ and $L_{X} \pi=0$, then $X=0$. Then in a neighborhood of $(g, \pi), \mathscr{C}_{g}^{z}$ is a smooth submanifold of $T^{*} . \|^{3}$, and $\delta_{g} \pi=0$ is linearization stable at $(g, \pi)$.

ProOf. $D \delta(g, \pi): S_{2}^{z} \times\left(S_{s}^{2} \otimes \mu_{g}\right) \rightarrow \mathscr{X}^{s-1} \otimes \mu_{R}$ is computed to give

$$
\beta_{(g, x)} \cdot(h, \omega)=D \delta(g, \pi) \cdot(h, \omega)=\delta \omega+\frac{1}{2} \pi^{d m} h_{l m}^{\mid i}-\pi^{l m} h_{l \mid m}^{i}
$$


with $L_{g}$ adjoint $\beta_{(5, \pi)}^{*}: \mathscr{X}^{s} \otimes \mu_{\mathrm{g}} \mapsto S_{2}^{s-1} \times\left(S_{s-1}^{2} \otimes \mu_{g}\right)$ given by

$$
\beta_{(s . x)}^{*}\left(X_{f^{\prime} g}\right)=\left(-\frac{1}{2}\left(L_{X} \pi+X \otimes \delta \pi+\delta \pi \otimes X\right)^{\prime b}, \frac{1}{2}\left(L_{X g}\right)^{=} \mu_{g}\right)
$$

( $\left(L_{X} \pi\right)^{\prime}$ means the tensor part of $\left.L_{X} \pi\right)$. Thus, since $\delta \pi=0, \beta_{(g, x)}^{*}\left(X \mu_{g}\right)=0 \Rightarrow$ $L_{X} \pi=0$ and $L_{X g}=0 \Rightarrow X=0$, so $\beta_{(g, x)}^{*}$ is injective. Also, $\beta_{(g, x)}^{*}$ has injective symbol (which it inherits from the second factor alone), so that $\beta_{(g, x)}=D \delta(g, \pi)$ is surjective and $\check{c}(\cdot, \cdot)$ is a submersion at $(g, \pi)$.

For $(g, \pi) \in T^{*}: \mathscr{H}^{s}$, let $I_{s}=\left\{\varphi \in \mathscr{Q}^{3+1}: \varphi^{*} g=g\right\}$, the isometry group of $g$, and $J_{x}=\left\{\varphi \in \mathscr{C}^{s+1}: \varphi^{*} \pi=\pi\right\}$, the symmetry group of $\pi$ (here $\varphi^{*} \pi=\left(\left(\varphi^{-1}\right)\right.$. $\left.\pi^{\prime}\right) \otimes$ $\left(\varphi^{*} \mu_{g}\right)$ is the pulltack of the contravariant tensor density $\left.\pi\right) . I_{g}$ is a compact Lie group; $I_{x}$ is clcsc $c$ in $: 2 s: 1$ but may be infinite-dimensional (e.g., if $\pi=0, I_{x}=$ $\mathscr{g}^{s+1)}$. Let $I_{(g,-1}=I_{g} \cap J_{z}$, a compact Lie group, and let $\mathscr{I}_{(g, x)}$ denote its Lie algebra. Then ker $\left.f^{\prime}, x, x\right)=\mathscr{F}_{(x, \pi)}=\left\{X \in \mathscr{Q}^{+1}: L_{X g}=0\right.$ and $\left.L_{X} \pi=0\right\}$.

If we consider tr: $:$ action $A: \mathscr{H}^{\text {stl }} \times \mathscr{H}^{3} \rightarrow \mathscr{H}^{3}$ lifted to the cotangent bundle,

$$
A^{\prime}: Q^{s+1}>T^{*} . \mathscr{H}^{s} \rightarrow T^{*} \cdot \mathscr{H}^{s}, \quad(\varphi,(g, \pi)) \mapsto\left(\varphi^{*} g, \varphi^{*} \pi\right)
$$

then the isotropy group of this action at a point $(g, \pi) \in T^{*} \cdot M^{s}$ is $I_{(g, x)}$. Thus the map $\delta(g, \pi)$ is singular (i.e., fails to have surjective derivative) precisely where the action $A^{\prime}$ has isctropy greup $I_{(g, z)}$ which is nondiscrete. At these $(g, \pi), \mathscr{D}^{*+1}$ does

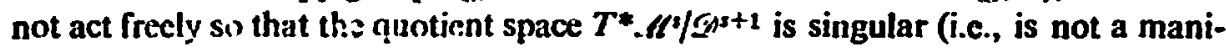
fold).

Note that if $(g, \pi) \cong \mathcal{C}_{\delta}^{s}$, nulling back $\delta_{g^{2}} \pi=0$ by $\varphi \in \mathscr{D}^{\text {s+1 }}$ gives $\varphi^{*}\left(\delta_{g} \pi\right)=$ $\delta_{s^{*} g}\left(\varphi^{*} \pi\right)=0$ so that $\left(\varphi^{*} g, \varphi^{*} \pi\right) \in \mathscr{C}_{\delta}^{*}$. Thus $\mathscr{C}_{\delta}$ is invariant under $A^{\prime}$ so that we have restricted action

$$
B: \mathscr{Q}^{s+1} \times \mathscr{C}_{\delta}^{s} \rightarrow \mathscr{C}_{\delta}^{s} .
$$

Let $\mathscr{E}_{d}^{J} / \mathscr{O}^{s+1}$ detote the quotient space of $\mathscr{E}_{j}^{\prime}$ by this restricted action. Because the singular points $(g, \pi)$ of $\mathscr{E}_{g}^{\prime}$ (as a manifold) correspond to singularities in the action $B$, and at these points $(g, \pi)$, $\operatorname{ker} \beta_{(g, x)}^{*}=\mathcal{F}_{(g, x)}$, we conjecture that modulo the presence of discrete isotropy groups, $\mathscr{E}_{\delta}^{*} / \mathscr{P}^{r+1}$ is a smooth submanifold as a subset of the quotient space $T^{*} \mathcal{M}^{s} / \mathscr{P}^{s+1}$, the singular points of $\mathscr{S}_{\delta}^{3}$ precisely "cancelling out" the singularities in the quotient space $\mathscr{C}_{b}^{J} / \mathscr{D}^{\text {s+1 }}$ due to the presence of nondiscrete isotropy. This possibility was pointed out to us by D. Ebin; cf. Marsden and Fischer [28].

At those points $(g, \pi) \in \mathscr{C}_{\delta}^{s}$ for which $D \delta(g, \pi)$ is not surjective, there are extra second order conditionș that must be satisfied for a deformation $(h, \omega)$ to be tangent to a curve in $\mathscr{C}_{\S}$.

11.3.2. THEOREM. $\operatorname{Let}(g, \pi) \in \mathscr{C}_{3}^{3}, X \in \mathscr{Q}^{s+1}, X \neq 0$ such that $L_{X g}=0$ and $L_{X} \pi=$ 0 . Let $(g(\lambda), \pi(\lambda)) \in \mathscr{G}_{\delta}^{s}, \lambda \in(-c, c), c>0$, be a $C^{2}$ curve with $(g(0), \pi(0))=(g, \pi)$, and $\left(g^{\prime}(0), \pi^{\prime}(0)\right)=(h, \omega)$. Then $(h, \omega)$ satisfies

and

$$
D \delta(g, \pi) \cdot(h, \omega)=\delta \omega+\frac{1}{2} \pi^{i m} h_{l m}^{1 i}-\pi^{l m} h^{i} \|_{1 m}=0
$$

$$
\int h \cdot L_{X} \omega=0 .
$$


Proof. Differentiating $\delta(g(\lambda), \pi(\lambda))=0$ twice and evaluating at $\lambda=0$ gives

$$
\left.\frac{d \delta}{d \lambda}(g(\lambda), \pi(\lambda))\right|_{\lambda=0}=D \delta(g, \pi) \cdot(h, \omega)=0,
$$

and

$$
\begin{aligned}
\left.\frac{d^{2} \delta}{d \lambda^{2}}(g(\lambda), \pi(\lambda))\right|_{\lambda=0}= & D^{2} \delta(g, \pi) \cdot((h, \omega),(h, \omega)) \\
& +D \delta(g, \pi) \cdot\left(g^{\prime \prime}(0), \pi^{\prime \prime}(0)\right)=0 .
\end{aligned}
$$

Thus, if $\operatorname{ker} \beta_{(g, \pi)}^{*} \neq 0$, then for each $X \in \operatorname{ker} \beta_{(g, \pi)}^{*}$,

$$
\begin{aligned}
\int X \cdot\left(D^{2} \delta(g, \pi)\right. & \cdot((h, \omega),(h, \omega)))+\int X \cdot\left(\beta_{(g, \pi)} \cdot\left(g^{\prime \prime}(0), \pi^{\prime \prime}(0)\right)\right) \\
& =\int X \cdot\left(D^{2} \delta(g, \pi) \cdot((h, \omega),(h, \omega))\right)+\int\left(\beta_{(g, \pi)}^{*} X\right) \cdot\left(g^{\prime \prime}(0), \pi^{\prime \prime}(0)\right) \\
& =\int X \cdot\left(D^{2} \delta(g, \pi) \cdot((h, \omega),(h, \omega))\right)=0 .
\end{aligned}
$$

$A$ rather lengthy computation gives

$$
\begin{aligned}
D^{2} \delta(g, \pi) \cdot((h, \omega),(h, \omega)) & \\
= & 2 \omega^{l m}\left(\frac{1}{2} h_{l m}^{l i}-h_{l \mid m}^{i}\right)-2 \pi^{i m} h^{\text {ia }}\left(\frac{1}{2} h_{l m \mid a}-h_{a l l m}\right),
\end{aligned}
$$

which, together with $D \delta(g, \pi) \cdot(h, \omega)=0$, gives

$$
\int X \cdot\left(D^{2} \delta(g, \pi) \cdot((h, \omega),(h, \omega))\right)=-\int L_{X \omega} \cdot h=0 .
$$

Thus, $\int L_{x} \omega \cdot h=0$ is the necessary second order condition for each $X \in \operatorname{ker} \beta_{(g, \pi)}^{*}$ that must be satisfied for $(h, \omega)$ to be tangent to a curve in $\mathscr{B}_{\%}$.

Thus at points $(g, \pi)$ of linearization instability of the equation $\delta_{g} \pi=0$, there is an extra condition generated by each $X \in \operatorname{ker}(D \delta(g, \pi))^{*}=\left\{X \in \mathscr{X}^{3}: L_{X} g=0\right.$ and $\left.L_{X} \pi=0\right\}$, so that the number of linearly independent extra conditions is equal to dim $\operatorname{ker}(D \delta(g, \pi))^{*}$. For the Hamiltonian constraint there was one extra second order condition, corresponding to the fact that $\operatorname{dim} \operatorname{ker}\left(D \mathscr{H}\left(g_{F}, 0\right)\right)^{*}=1$.

I1.4. The constraint manifold $\mathscr{C}^{s}$. We now consider the constraint set $\mathscr{C}^{s}=\mathscr{C}_{x}^{3} \cap$ $\mathscr{C}_{\mathrm{g}}^{\mathrm{j}}$. To show that $\mathscr{C}^{s}$ is a submanifold of $T^{*} \mathscr{M}^{*}$, we need additional restrictions in order to ensure that the intersection is transversal. At this point it is necessary to assume that $\operatorname{tr} \pi=$ constant. (See Note on p. 263.)

I1.4.1. TheOREM. Let $(g, \pi) \in \mathscr{C}^{s}=\mathscr{C}_{x}^{s} \cap \mathscr{C}_{\delta}^{3}$ satisfy the following conditions:

$$
\begin{aligned}
& C_{X}: \text { if } \pi=0, g \text { is not flat; } \\
& C_{d}: L_{X}=0 \text { and } L_{X} \pi=0 \Rightarrow X=0 ; \\
& C_{\mathrm{tr}}: \operatorname{tr} \pi^{\prime}=\text { constant. }
\end{aligned}
$$

Then, in a neighborhood of $(g, \pi), \mathcal{C}^{s}$ is a $C^{\infty}$ submanifold of $T^{*} \mathscr{M}^{s}$.

Proof. Let $\Psi=(\mathscr{H}, \delta): T^{*} \mathscr{H}^{s} \rightarrow \Lambda^{s-2} \times\left(\mathscr{X}^{s-1} \otimes \mathscr{V}^{s}\right),(g, \pi) \mapsto(\mathscr{H}(g, \pi), \delta(g, \pi))$.

Then

$$
\begin{aligned}
D \Psi(g, \pi) \cdot(h, \omega) & =(D \mathscr{H}(g, \pi) \cdot(h, \omega), D \delta(g, \pi) \cdot(h, \omega)) \\
& =\left(\gamma(g, \pi) \cdot(h, \omega), \beta_{(g, \pi)} \cdot(h, \omega)\right)
\end{aligned}
$$


and the $L_{2}$-adjoint of $D \Psi(g, \pi)$ is

$$
(D /(g, \pi))^{*} \cdot\left(N \mu_{g}, X \otimes \mu_{g}\right)=\gamma_{(g, \pi)}^{*}\left(N \mu_{g}\right)+\beta_{(g, \pi)}^{*}\left(X \mu_{g}\right) .
$$

Thus suppose $(D \mathscr{U}(g, \pi))^{*}\left(N \mu_{g}, X \otimes \mu_{g}\right)=0$. Then

$$
\begin{aligned}
g J N & + \text { Hess } N-N \operatorname{Ric}(g)+\frac{1}{2} N R(g) g \\
& \left.+2\left(\frac{1}{2}\left(\operatorname{tr} \pi^{\prime}\right) \pi^{\prime}-\pi^{\prime} \times \pi^{\prime}\right)^{b}-\frac{1}{2}\left(\frac{1}{2}\left(\operatorname{tr} \pi^{\prime}\right)^{2}-\pi^{\prime} \cdot \pi^{\prime}\right) g\right\} N \\
& -\frac{1}{2}\left(L_{X} \pi+X \otimes \delta \pi+\delta \pi \otimes X\right)^{\prime 2}=0 ;
\end{aligned}
$$

$$
2\left(\frac{1}{2}(\operatorname{tr} \pi) g-\pi\right) N+\frac{1}{2}\left(L_{X} g\right)^{4} \mu_{g}=0 .
$$

Taking the trace of (a) and using $\mathscr{H}(g, \pi)=0$ and $\delta \pi=0$ gives

$$
2 J N-\frac{1}{2}\left(X \cdot d \operatorname{tr} \pi^{\prime}-\pi^{\prime} \cdot L_{X} g-(\delta X)\left(\operatorname{tr} \pi^{\prime}\right)\right)=0,
$$

and from the trace of $(b)$,

$$
\delta X=N \operatorname{tr} \pi^{\prime}
$$

From (b),

$$
L_{X} g=4\left(\pi-\frac{1}{2}(\operatorname{tr} \pi) g\right) N,
$$

and, subsituting (d) and (e) into (c) gives

$$
2 \Delta N+2\left(\pi^{\prime} \cdot \pi^{\prime}-\frac{1}{4}\left(\operatorname{tr} \pi^{\prime}\right)^{2}\right) N-\frac{1}{2} X \cdot d \operatorname{tr} \pi^{\prime}=0 .
$$

Since $P\left(\pi^{\prime}, \pi^{\prime}\right) \equiv \pi^{\prime} \cdot \pi^{\prime}-\frac{1}{1}\left(\operatorname{tr} \pi^{\prime}\right)^{2}=\left(\pi^{\prime}-\frac{1}{2}\left(\operatorname{tr} \pi^{\prime}\right) g\right) \cdot\left(\pi^{\prime}-\frac{1}{2}\left(\operatorname{tr} \pi^{\prime}\right) g\right)$, the coefficient of $N$ is positive-definite. Thus, if tr $\pi^{\prime}=$ constant,

$$
2 \Delta N+2 P\left(\pi^{\prime}, \pi^{\prime}\right) N=0,
$$

so that $N=0$ unless $\pi^{\prime}=0$. If $N=0$, from (a) $L_{X} \pi=0$ and from (b) $L_{X} g=0$ so $X=0$.

If $\pi^{\prime}=0$, then $\Delta N=0$ so $N$ is constant and so from (a), $N\left(\operatorname{Ric}(g)-\frac{1}{2} g R(g)\right)$ $=0 \Rightarrow N=0$, since $\operatorname{Ric}(g) \neq 0$ in the case that $\pi^{\prime}=0$. Then, again, $X=0$. Thus, in either case, $(D \Psi(g, \pi))^{*}$ is injective.

The symbol $\sigma_{\xi_{0}}\left(D \Psi(g, \pi)^{*}\right)$ is given by

$$
\begin{array}{r}
\sigma_{\xi} \cdot(s, Y)=\left\{\left(-g\left\|\xi_{x}\right\|^{2}+\xi_{x} \otimes \xi_{x}\right) s-\frac{1}{2}\left(-\pi_{i}^{\prime k} \xi_{k} Y_{j}-\pi_{j}^{j k} \xi_{k} Y_{i}+\pi_{i j}^{\prime} \xi_{k} Y^{k}\right),\right. \\
\left.\frac{1}{2}\left(Y \otimes \xi_{x}^{\xi}+\xi_{x}^{k} \otimes Y\right) \mu_{g}(x)\right\} .
\end{array}
$$

Thus if $\sigma_{\xi}(s, Y)=0, \xi_{x} \neq 0$, from the second factor $Y=0$ and so from the first factor $s=0$. Thus $\sigma_{\xi}$, is injective, $D \mathscr{V}(g, \pi)$ is surjective, and $\Psi$ is a submersion at $(g, \pi)$.

It would be nice if the tr $\pi^{\prime}=$ constant condition could be dropped and we conjecture that it can. However, because of the coupling of equations (a) and (b) without the $\operatorname{tr} \pi^{\prime}=$ const condition, it is possible that these equations have nonzero solutions $(N, X)$ even at those $(g, \pi)$ that satisfy conditions $C_{\boldsymbol{x}}$ and $C_{\delta}$.

II.5. Integrating deformations of Ricci-flat spacetimes. As explained previously, we can use II.4.1 to prove the following result. We consider only the $C^{\infty}$ case here.

II.5.I. THEOREM. Let ${ }^{(4)} g$ be a smooth Lorentz metric on a 4-manifold $V$ satisfying 
Einstein's empty-space field equations $\operatorname{Ric}\left({ }^{(4)} g\right)=0$, and let ${ }^{(4)} h$ be a solution to the linearized equation

about the solution ${ }^{(1)} \mathrm{g}$.

$$
D \operatorname{Ric}(\omega) g) \cdot(1) h=0
$$

\&Assume that $\boldsymbol{V}$ has a compact connected oriented spacelike hypersurface $\boldsymbol{M}$ with induced riemannian metric $g$ and second fundamental form $k$ that satisfy conditions $C_{x}, C_{\delta}$, and $C_{\mathrm{t}}$. Then there exist a tubular neighborhood $V^{\prime}$ of $M, a \delta>0$, and $a$ smooth curve (4) $g(\lambda),-\delta<\lambda<\delta$, of exact solutions to Einstein's equations defined on $V^{\prime}$ tangent to ${ }^{(4)} h$ at ${ }^{(4)} g$, i.e., ${ }^{(4)} g(0)={ }^{(4)} g,{ }^{(1)} g^{\prime}(0)={ }^{(4)} h$, and $\operatorname{Ric}((1) g(\lambda))=0$ in a tubular neighborhood of $M$.

Proof. Let $(g, \pi)$ be the variables on $M$ induced by ${ }^{(4)} g$. A deformation ${ }^{(4)} h$ of $\operatorname{Ric}\left({ }^{(4)} g\right)=0$ induces a deformation $(h, \omega)$ of the linearized constraint cquations, $D \mathscr{H}(g, \pi) \cdot(h, \omega)=0, D \delta(g, \pi) \cdot(h, \omega)=0$. Since $(g, \pi)$ satisfies conditions $C_{x}, C_{\delta}$, and $C_{t r}$ by II. $4.1, \mathscr{C}$ is a smooth submanifold with tangent space $T^{(g . x)} \mathscr{C}$ $=\operatorname{ker}(D \mathscr{H}(g, \pi), D \delta(g, \pi))$. Since $(h, \omega)$ is tangent to $\mathscr{E}$, we can find a curve $(g(\lambda), \pi(\lambda)) \in \mathscr{B}$ tangent to $(h, \omega)$. By the evolution theory, this curve of solutions to the constraint equations gives us a curve ${ }^{(1)} g(\lambda)$ of spacetimes defined in a tubular neighborhood $V^{\prime}$ of $M$. By a transformation of coordinates, ${ }^{(4)} g(\lambda)$ can be made tangent to ${ }^{(4)} h$. See [16] for details.

Thus a solution of the linearized Einstein empty-space field equations actually approximates to first order a curve of exact solutions to the nonlinear equations in a tubular neighborhood of any compact spacelike hypersurface that satisfies conditions $C_{\boldsymbol{x}}, C_{j}$, and $C_{t r}$. Because these conditions are so weak, presumably most spacetimes which have compact spacelike hypersurfaces have a hypersurface $M$ satisfying these conditions, and thus is linearization stable in a tubular neighborhood of $M$. Moreover, by using standard arguments and by considering the maximal development (see [12D) of the Cauchy data of the curve of spacetimes ${ }^{(4)} g(\lambda)$, there will be a maximal common development (which approximates the maximal development of ${ }^{(4)} g(0)$ ) for which the spacetime is linearization stable.

\section{REFERENCES}

1. R. Amowitt, S. Deser and C. W. Misner, The dynamics of general relativity, in Gravitation: An Iurroduction to Current Research (L. Witten, Ed.), Wiley, New York, 1962, pp. 227-265. MR 26 \#1182.

2. M. Berger, Sur les variétés d'Einstein compactes, C. R. de la III' Réunion du Groupement des Mathématiciens d'Expression Latine (Namur, 1965), Librairie Universitaire, Louvain, 1966, pp. 35-55. MR 38 \#6502.

3. - Quelques formules de veriation, pour whe structure riemannienure, Ann. Sci. Ecole Norm. Sup. (4) 3 (1970), 285-294. MR 43 \#3969.

4. M. Berget and D. Ebin, Some decompasitions of the space of symmetric tensars on a Riemannian manifold, J. Differential Geometry 3 (1969), 379-392. MR 42 \#993.

5. D. Brill, Isolated solutions in general relativity, in Gravilation: Problems and Prospects. "Naukova Dumka", Kiev, 1972, pp. 17-22.

6. D. Brill and S. Deser, Instability of closed spaces in general relativity, Comm. Math. Phys. 32 (1973), 291-304. 
7. Y. Choquet-Brubat, New elliptic system and global solutions for the constraines equations in general relativity, Comm. Math. Phys. 21 (1971), 211-218.

8. - Global solutions of the equations of constraint in general relasivity on closed manifolds, Istituto Nazionale di Alta Matematica, Symposia Matematica, vol. XII, Bologna, Academic Press, New York, 1973.

9. - Global solutions of the constraints equations on open and closed manifolds, General Relativity and Gravitation 5 (1974), $49-60$.

10. Y. Choquet-Bruhat and S. Deser, On the stablity of fiat space, Ann. Phys. 81 (1973), 165-178.

11. - Stabilité intitale de l'espace temps de Minkowski, C. R. Acad. Sci. Paris Sér. A-B 275 (1972), 1019-1021.

12. Y. Choquet-Bruhat and R. Geroch, Global aspects of the Cauchy problem in general relativity, Comm. Math. Phys. 14 (1969), 329-335. MR 40 \#3872.

13. D. Ebin, The manifold of Riemamitan metrics, Proc Sympos. Pure Math., vol. 15, Amer. Math. Soc., Providence, R.I., 1970, pp. 11-40; see also: Bull. Amer. Math. Soc. 74 (1968), $1002-$ 1004. MR 42 \#2506.

14. A. Fischer and J. Marsden, The Einstein equations of exolution-a geometric approach, J. Mathematical Phys. 13 (1972), 546-568. MR 45 \#8214.

15. - The Einstein evolution equations as a first-order quasi-linear symmetric hyperbolic system. I, Comm. Math. Phys. 28 (1972), 1-38. MR 46 \#8616.

16. Linearization stability of the Einstein equations (to appear); see also: Bull. Amer. Math. Soc. 79 (1973), 997-1003.

17. - Global analysis and general relativity, General Relativity and Gravitation 5 (1973), $73-77$.

18. _- Deformations of the scalar curvature, Duke Math. J. (to appear); see also: Bull. Amer. Math. Soc. 80 (1974), 479-484.

19. A. Fischer and J. A. Wolf, The Calabi construction for compact Riccl-fat rlemannitan montfolds, Bull. Amer. Math. Soc. 80 (1974), 92-97.

20. - The structure of compact Ricci-flat riemannian manifolds, J. Differential Geometry 10 (1975).

21. J. Kazdan and F. Warner, Prescribing curratures, these Procrzonicas.

22. - A direct approach so the determination of Gouscian and scalor curpature functions, Invent. Math. (to appear).

23. S. Lang, Differential manifolds, Addison-Wesley, Reading, Mass, 1972.

24. A. Lichnerowicn, Lintégration des équations de la gravitation relativiste et le probleme des n corps, J. Math. Pures Appl. (9) 23 (1944), 37-63. MR 7, 266.

25. - Propagateurs et commutateurs en relativité générale, Inst. Hautes Études Sci. Publ. Math. No. 10 (1961), 56 pp. MR 28 \$967.

26. - Spineurs harmoniques, C. R. Acad. Sci. Paris 257 (1963), 7-9. MR 27 \#6218.

27. J. Marsden, D. Ebin and A. Fischer, Diffeomeophism groups, hydrodyncamics, and general relativtty, Proc. Thirteenth Biennial Seminar of the Canadian Math. Congress, Montreal, 1972. pp. 135-279.

28. J. Marsden and A. Fischer, General relativity as a Hamilronian system, Istituto Nazionalodi Alta Malematica, Sýmposia Matematica XIV, Bologna, Academic Press, New York, 1974, pp. 193-206.

29. E. Nelson, Tensor analysis, Princeton Univ. Press, Princeton, N.J., 1967.

30. L. Nirenberg and H. Walker, The mull spaces of elliptic partial differential operators in $\boldsymbol{R}^{\text {. }}$, J. Math. Anal. Appl. 42 (1973), 271-301.

31. M. Obata, Certain condilions for a riemannian manifold to be isometric with a sphere, J. Math.

Soc. Japan 14 (1962), 333-340. MR 25 \#5479.

32. - Riemannian manifolds admitting a solution of a certain system of differential equarions, Proc. United States-Japan Seminar in Differential Gcometry (Kyoto, 1965), Nippon Hyoronsha, Tokyo, 1966, pp. 101-114, MR 35 \#7263. 
33. N. O'Murchadha and J. W. York, Existence and uniqueness of solutions of the Hamiltonion constraint of general relativity on compact manifolds, J. Mathematical Phys. 14 (1973), $1551-1557$.

34. K. Yano, On harmonic killing vectors, Ann. of Math. (2) 55 (1952), 38-45. MR 13, 689.

35. J. A. Wolf, Spaces of constant curvature, 2nd ed., J. A. Wolf, Berkeley, Calif., 1972.

36. - Local and global equivalence for flat affine manifolds with parallel geometric structures, Geometriae Dedicata 2 (1973), 127- 132.

37. J. P. Bourguignon, Thèse, Université de Paris VII, 1974; also, Compositio Math. (to appear). 38. Y. Muto, On Einstein metrics, J. Differential Geometry 9 (1974), 521-530.

39. V. Moncrief, Spacetime symmetries and linearization stability of the Einstein equations, J. Math. Phys. (to appear).

40. P. D'Eath, Three perturbation problems in general relativity, Thesis, Cambridge, 1974.

Note. In [39], Moncrief shows that the map $\Psi$ in 11.4 .1 is a submersion if and only if $V$, the spacetime, has no killing fields. Thus the conditions on p. 248 can be replaced by " $V$ has no killing fields." For some applications to specific spacetimes, see [40].

Universtry of California, Santa Cruz and Berkeley 